\title{
End-to-End Performance of ATM and Private Broadband Networks
}

\author{
Arne A. Nilsson
}

Mario Huterer

Center for Communications and Signal Processing Department of Electrical and Computer Engineering North Carolina State University

\author{
TR-91/21 \\ December 1991
}




\section{TECHNICAL REPORT}

\section{End-to-End Performance of ATM and Private Broadband Networks}

Arne Nilsson and Mario Huterer

Center for Communications and Signal Processing Department of Electrical and Computer Engineering North Carolina State University Raleigh, NC 27695 - 7914 


\section{ABSTRACT}

In this report we describe a simulation study on the four different network topologies which include networks with fixed and variable cell sizes. In the first two topologies we evaluate the end-to-end packet delay of the fixed, 53-bytes, cell-size network (ATM) and the variable cell-size network. In the last two topologies we obtain packet delay results for the interconnected variable and fixed cell-size networks. Particular emphasis is given to evaluating various approaches for transmitting the ATM cells over a variable cell-size network with respect to minimizing the average end-toend packet delay.

Our models include the Poisson and the Markov Modulated Poisson arrival Process (MMPP) and several packet length distributions. We also show results reflecting the buffer requirements in the network nodes. The results for each topology are discussed separately and compared against each other. We emphasize that end-to-end packet delay depends significantly on the burstiness of the network traffic and modeling of bursty arrivals is essential. 


\section{TABLE OF CONTENTS}

1. INTRODUCTION ...............................................................

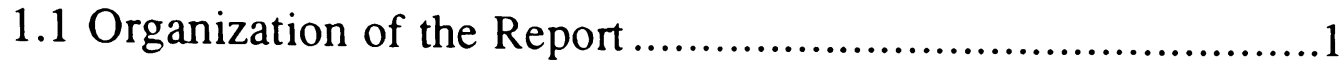

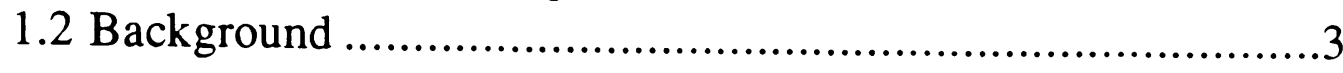

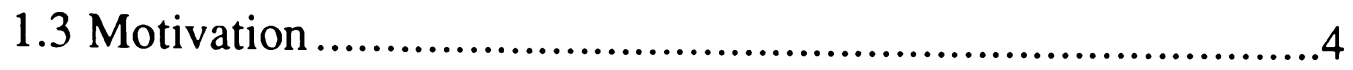

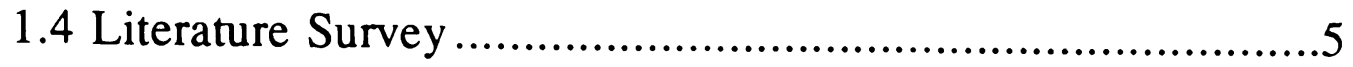

2. END-TO-END MODELS AND ASSUMPTIONS ............................7

2.1 ATM and Megacell Network Models ...................................9

2.2 Cell Formats in ATM and Megacell network ........................11

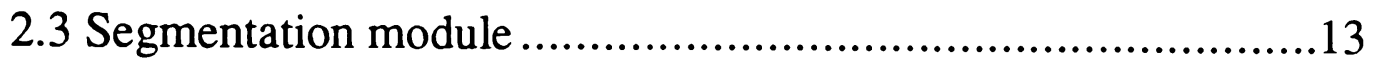

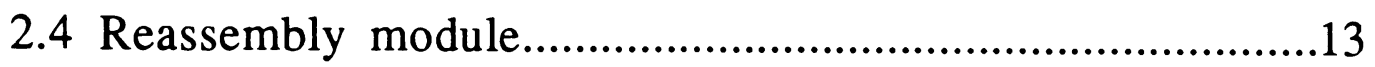

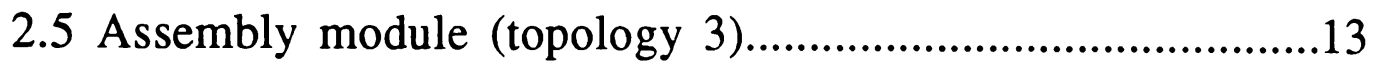

2.6 Modeling of Packet Retransmissions ....................................14

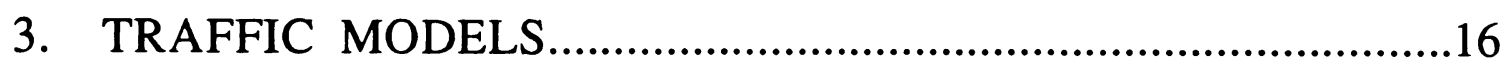

3.1 Burstiness in Broadband Networks .......................................16

3.2 Superposition of the Arrival Processes.....................................17

3.3 Validity of a Poisson Process in Broadband Networks .............18

3.4 Markov Modulated Poisson Process (MMPP) ….....................20

3.5 Modeling the VC- and External Traffic-Arrivals with

Markov Modulated Poisson Process ............................................21

3.6 Characterization of Burstiness of the Arrival Process.............22

3.7 Packet Length Distributions..................................................24

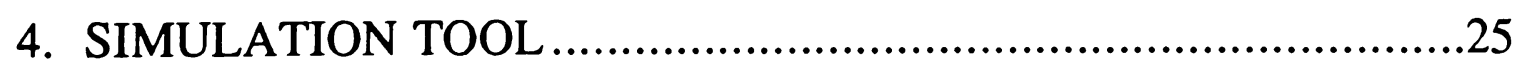

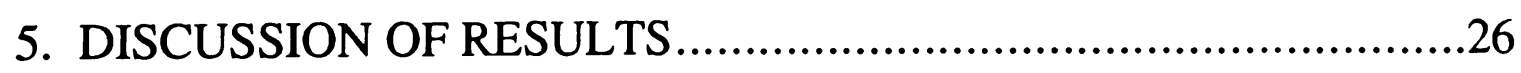

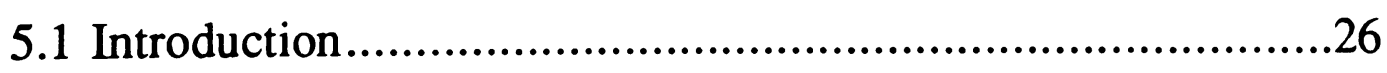

5.1.1 The focus of the study .........................................26

5.1.2 The choice of the assumptions for the studies..............26

5.1.3 Outline of Assumptions for Model Parameters............27

5.1.4 Plotting of End-to-end Delay Graphs ........................29

5.1.5 Accuracy of the simulation results ............................29 


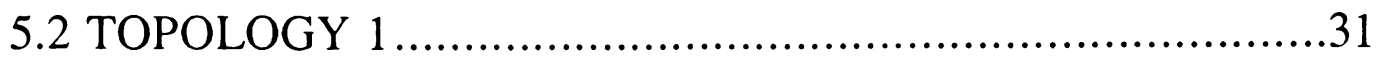

5.2.1 Block Diagram ....................................................

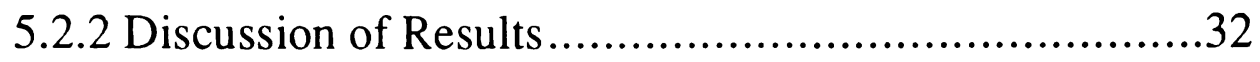

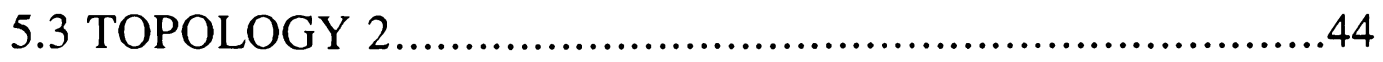

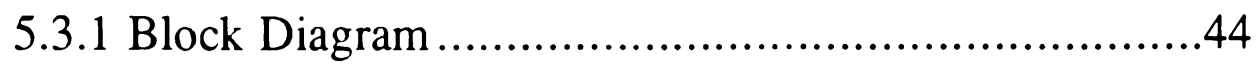

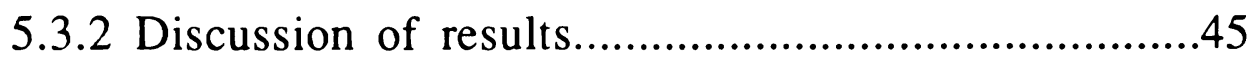

5.4 TOPOLOGY 1 AND 2

A COMPARISON

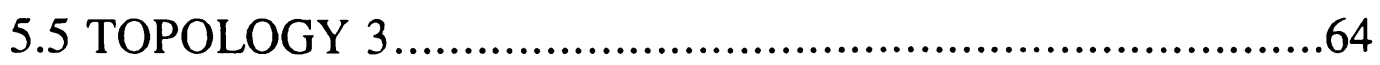

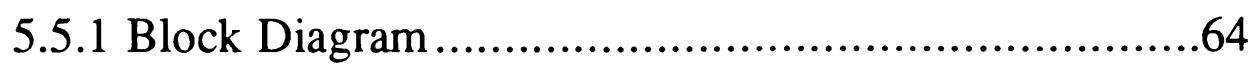

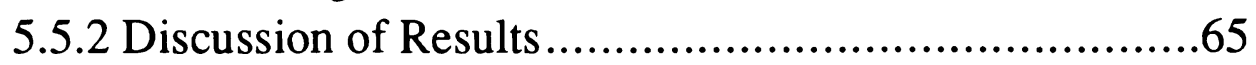

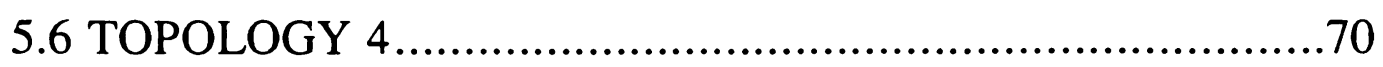

5.6.1 Block Diagram ...................................................... 70

5.6.2 Discussion of Results.............................................71

5.7 TOPOLOGY 1 - 4

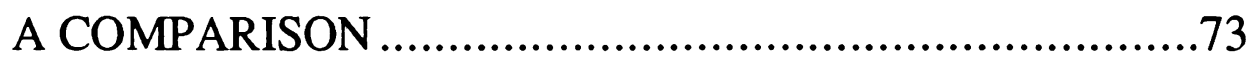

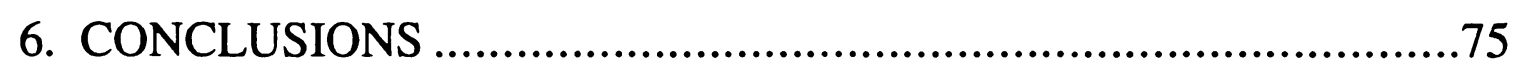

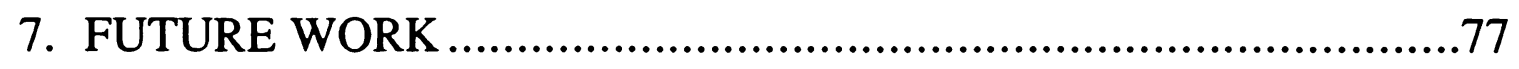

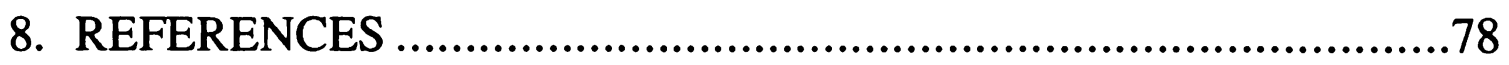

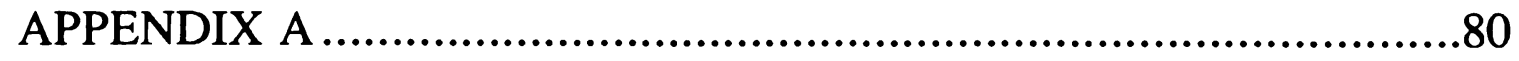

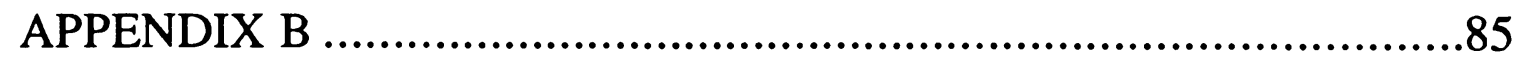

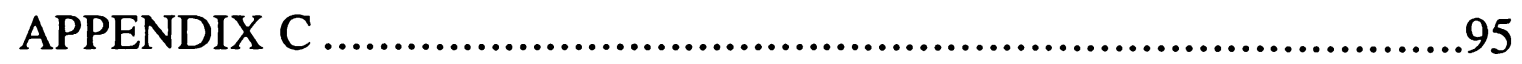

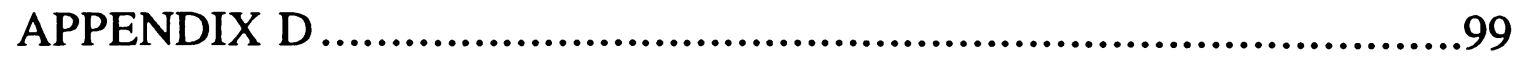




\section{INTRODUCTION}

\subsection{Organization of the Report}

Chapter 1 is the Introduction and includes background information about the broadband environment, the motivation for studying fixed and variable cell size high-speed networks and an overview of related papers.

In Chapter 2 we present the four end-to-end network topologies under study in this report and describe individual network components including the models for the fixed cell-size (the 'ATM') and variable cell-size (the 'Megacell') network and the Segmentation, Reassembly and Assembly modules. We also discuss here the overhead structure in the ATM and Megacell network and explain the implementation of packet retransmissions in the models.

Chapter 3 discusses the arrival processes and the packet length distributions used in the models. We stresses the importance for modeling of bursty and correlated inter-arrival times which characterize traffic in B-ISDN networks and discuss in more detail the Markov Modulated Poisson Process. Several methods for characterizing 'burstiness' are presented.

In Chapter 4 we discuss briefly the simulation tool - QNAP2, used in generating the results in this study.

Chapter 5 is the discussion of the results. We present the numerical values for the parameters used in the models and explain the reasons for our decision concerning the choice of the assumptions in the end-to-end models.

The discussion of the results is organized as follows:

- Section 5.2: Topology1

- Section 5.3: Topology2

- Section 5.4: $\quad$ Comparison of the Topologies 1 and 2

- Section 5.5: Topology3

- Section 5.6: Topology4

Chapter 6 is the Conclusions. Chapter 7 describes the future work.

Following the References are the Appendix A and B and C: 
In Appendix A we give a detailed description of the ATM and Adaptation layer, as specified in the CCITT B-ISDN standard document.

In Appendix B we present an extract of our results from the 'Study of the burstiness of the departure process for the superposition of various arrival processes and packet-length distributions'.

In Appendix $\mathrm{C}$ we present our derivation of the Laplace transform of the Inter-arrival times and the Squared Coefficient of Variation $\left(c^{2}\right)$ for an MMPP process.

In Appendix D we derive an approximate result for the square coefficient of variation of inter-departure times for an MMPP/M/1 queue. 


\subsection{Background}

The goal of BISDN is to provide a unique way of transmitting voice, data, and video traffic on a common backbone network. A Broadband-ISDN standard should permit independently designed networks to be interconnected easily, thereby resulting in an ubiquitous worldwide service offerings. Large number of individual traffic sources can be multiplexed and an aggregate network throughputs of several Gigabit/s can be expected. The variety of traffic types and their distinct requirements pose numerous challenging research and design problems.

The quality of service that will be provided by a broadband network is affected by the cell loss probability and the end-to-end delay. It is anticipated that different classes of traffic will require different quality of service. In particular, voice and video are tolerant to cell loss but not to time delays. On the other hand, the transfer of data packets (e.g. bulk files) is tolerant to time delays but not to cell loss.

A unique size for the information units, cells, that are going to carry broadband traffic and satisfy the requirements for all the traffic types is very difficult to determine. Over the last years there has been a considerable debate on this issue. There are a number of constraints to be considered including the impact of delay on voice traffic, network delay, processing requirement for header information and the transmission efficiency. Clearly these constraints are contradictory and some of them point towards a desire for big cells, some towards small cells.

The CCITT SG XVIII committee, which is responsible for Broadband Integrated Services Data Networks (BISDN) standards, has defined the Asynchronous Transfer Mode (ATM) as a transport mechanism for BISDN and the ATM cell size to be 53 bytes long [1]. This cell size has not been agreed upon undesputedly and an alternative variable-length cell approach has been proposed. It is to be expected that both solutions are going to coexist in the future and these networks are going to be interconnected.

The Variable Length Cell (VLC) network appears as a viable alternative to the Fixed Length Cell (FLC) 53-byte ATM network. In the case of a VLC network we assume that the user packets are transmitted without being segmented into smaller units. Since the VLC-sizes are usually considerably bigger than the 53-byte ATM cells, we refer to those large information units as 'megacells' and the VLC network as the 'Megacell' network in this report. 
In the ATM network, however, user packets are segmented into cells and transported over the network to the destination. At the destination the cells are reassembled into packets and delivered to the user. This process is specified in the CCITT standard which is described in more detail in the Appendix A.

Both choices, the VLC and FLC networks, have their advantages and disadvantages. We are going to investigate the end-to-end delay performance of the two network types and their interconnections by studying the four network topologies shown in figure 1 .

\subsection{Motivation}

Broadband-ISDN is an evolving standard and there are still a number of unresolved issues. We have already indicated that the choice of the cell size has been a long debated issue. It should be expected that private high speed networks will not necessarily implement the 53 byte ATM cell-size standard. Different choices for packet length sizes may be made as a result of selecting cell sizes which are going to be optimal in the networking environment of the particular private networks. Therefore, when choosing the cell size for a private network, a performance comparison of the standard ATM public network (with the fixed 53 byte cell-size) versus private Variable Length Cell (VLC) networks is very important.

Private networks will coexist and be interconnected with public ATM networks. This raises a number of challenging problems dealing with interconnecting variable and fixed length size networks. One of the problems is going to be e.g. the optimal packetization of the ATM cells into larger units on the entry point to a VLC network. We are going to concentrate on these issues in topologies 3 and 4.

Similar problems arise when interfacing broadband networks to the existing Local Area Networks (LANs). One can envision that user packets will be carried by Local Area Networks (LANs), which will in turn access BISDN network for wide-area transport. Thus, studying the interface between BISDN (ATM or VLC network) and LANs, like e.g. the FDDI (Fiber Distributed Data Interface) [2] [3] [4], will be very important.

Packet sizes are generally expected to become longer in the future as users begin to take full advantage of wide-area, broadband networks. Many broadband applications are going to carry substantial amount of information. A digital image of an X-ray can represent up to five or even ten megabyte of information. This brings up further interesting 
investigation to determine how effectively certain applications, like a bulk image file transfer from the above example, can be transmitted over a VLC versus ATM network.

\subsection{Literature Survey}

Several previous studies, [5] [6] [7] [8] investigated performance of the FLC and VLC ATM networks.

In [5] Parekh and Sohraby studied ATM FLC versus VLC format based on the memory requirements, delays and efficiency considerations. A comparison of the two formats was done by modeling the access multiplexer. The arrival process for the VLC multiplexer model is assumed to be Poisson and the multiplexer is modeled as an M/G/1 queue. Arrival process for the FLC model is a Poisson Wave Process which is a special case of a branching Poisson process [9] and the multiplexer is modeled as a $\mathrm{M}[\mathrm{W}] / \mathrm{D} / 1$ queue. The authors indicate how to obtain an approximate result for calculating mean waiting time in the FLCmultiplexer queue, based on the results by Eckberg [10] [11].

Salahi [6] provides mainly a simulation study for a two node broadband network model and illustrates the performance at the adaptation and ATM layer for FLC and VLC ATM network. In the analytical model, the author uses $M / D / 1$ queues to calculate end-to-end delay for the FLC-format network. To account for the bursty arrival process, the arrival process is approximated by a renewal process, using the results from Whitt [12]. The first two moments of the inter-arrival time of a GI/G/1 queue are used in approximating the renewal process. Analytical results do not include endto-end performance at the adaptation layer. Simulation results indicate higher end-to-end packet delay for the VLC format.

Cidon, Derby et al. [7] of IBM provide a critique of ATM by comparing ATM FLC network to the 'PARIS' high speed wide-area networking project which has been in progress since 1986 in IBM Research and is essentially a VLC-type broadband network. Concerning the packet length distributions which are used in the analysis, authors refer to the sets of published measurements on Local Area Networks (LANs) and broadcast networks, like the MIT, Berkley and Delaware distributions, [13] [14] [15] respectively. The published packet-size distributions have been "stretched" in terms of packet lengths, since the authors assumed that packet lengths are generally going to increase in the future. 
The authors used quantitative evidence to show that ATM FLC format is not optimized for data transfer. VLC ATM network has significantly better performance characteristics. However, the paper does not show explicit analytical or simulation results concerning the packet delays.

In [8] De Simone and Saksena provide simulation study in which they show that the FLC network has superior end-to-end delay performance compared with the VLC datagram network with respect to the number of hops traversed. The justification for the lower delay in FLC type network is based on the 'pipelining' argument: In an N-node connection FLC network (ATM), the mean packet delay only increases in proportion to a cell transmission and processing delay, rather than a packet transmission and processing delay, as in the VLC network. As the network diameter increases, the ATM architecture will have increasingly superior delay performance. However, the result were obtained by assuming network utilizations of less than $50 \%$ and large processing delay at the network access-mutiplexer for the VLC network. 


\section{END-TO-END MODELS AND ASSUMPTIONS}

Our simulation models are based on the four network topologies, shown in figure 1. As indicated earlier, the four topologies are composed of interconnected Variable and Fixed Cell Size networks ('Megacell' and 'ATM' networks, respectively). We focus on studying the end-to-end delay performance for the packets traversing a three-hop Virtual Circuit Connection (VCC) in each topology.

topology 1

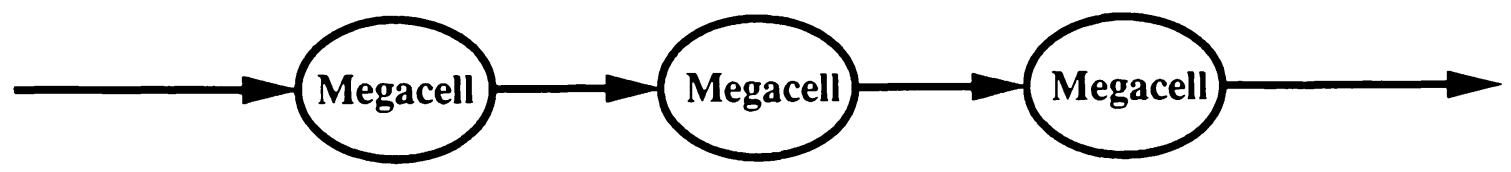

topology 2

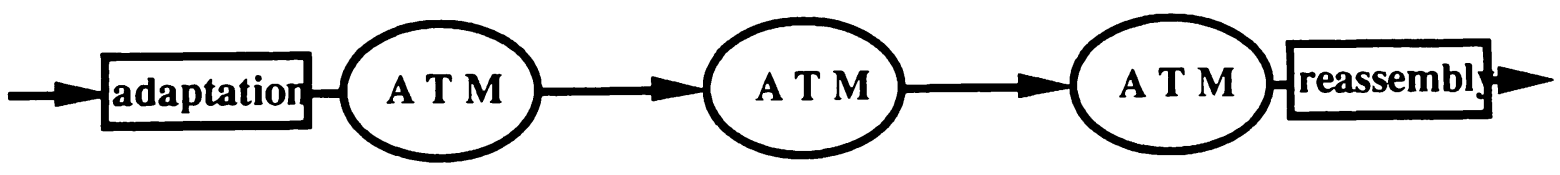

topology 3

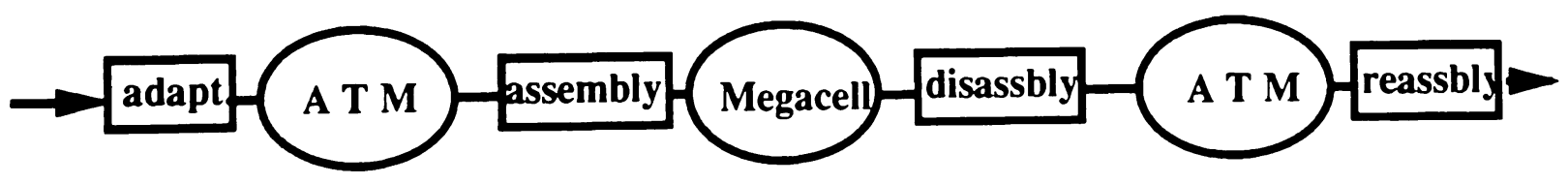

topology 4

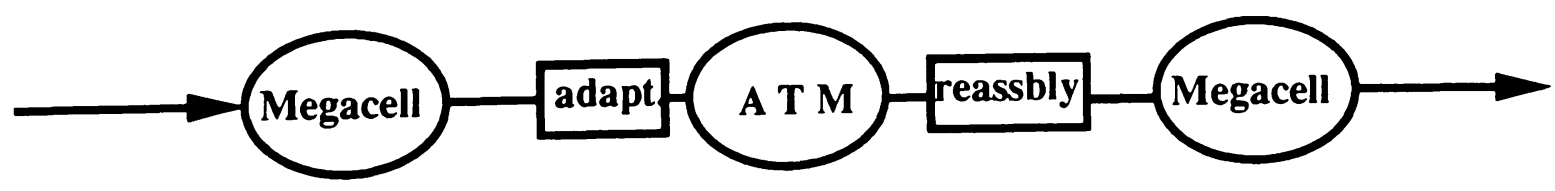

figure 1. Network Topologies 
In the first two topologies we chose the networks to be of the same type. In topology 1 all networks are of VLC type and in topology 2 of FLC type. This enables us to make comparisons of the VLC and FLC networks.

In topology 3 and 4, packets are being transmitted on a different type of network on each successive hop to the destination. Those two networks enable us to study end-to-end delay performance for the heterogeneous broadband networks and also study various interesting problems which arise in interconnecting heterogeneous networks.

Since we are interested in the end-to-end delay performance on the 'packet' level, in the case of an ATM network we include the models for the adaptation layer, namely, the 'Segmentation' module at the source and the 'Reassembly' module at the destination. In the case of a Megacell network, no adaptation layer is modeled since no segmentation of user packets is assumed.

In the topology 3, where an ATM network is connected to the Megacell network, we have assumed that the incoming ATM cells may be assembled into a single 'megacell', and, thereby reduce the amount of overhead transmitted over the Megacell network. This is done in the 'Assembly' module. The efficient way of assembling ATM cells in the Assembly is another important research issue in this study.

We provide a detailed overview of the ATM and Adaptation layer functions and the related header sizes, specified in the CCITT B-ISDN standard document [1], in Appendix A. This document was the basis for the assumptions that we made with respect to modeling the ATM network. For modeling the variable cell-size network (the 'Megacell' network) we followed the recommendations obtained from IBM and [7].

In the following sections we describe individual network components which constitute topologies 1-4 from figure 1. Later we discuss the issues concerning the overhead assumed in ATM and Megacell networks and explain the implementation of packet retransmissions in the models. 


\subsection{ATM and Megacell Network Models ${ }^{1}$}

Individual networks, Megacell and ATM (the ATM layer), are modeled as shown in figure 2 . We assume that all the nodes which are on the end-toend path for the user packets in a particular network may be represented by a single server queueing system.

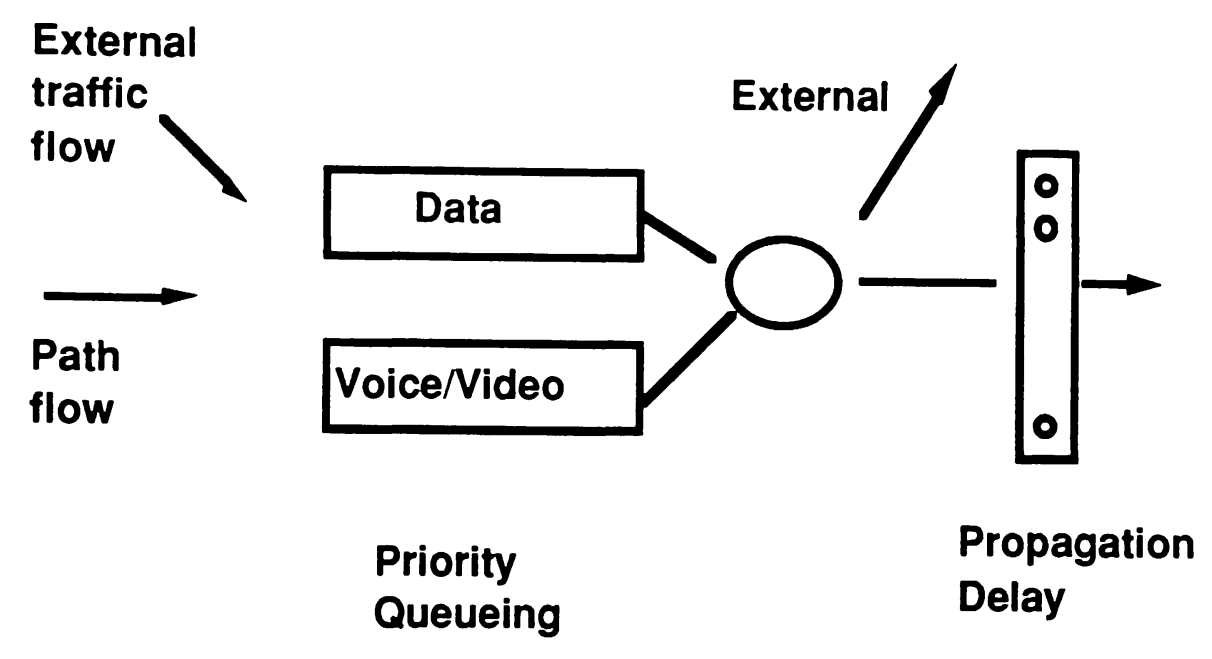

figure 2. Network model

Models for the ATM and Megacell network are the same except for the service time, which are constant in the ATM model and variable in the Megacell model.

\section{Arrivals - Virtual Circuit and External traffic:}

The traffic on the end-to-end $\mathrm{VCC}(\mathrm{s})$ (figure 1) is called the 'user' traffic or the 'VC-traffic' since the user establishes the VCC before transmitting the information to the destination. The user traffic is represented in figure 2 as a 'path flow' since the end-to-end VCC is composed of one or more Virtual Path (VP) connections between the successive networks nodes and all the user information follows the same path to the destination.

The 'VC-traffic' traffic interferes with the traffic from other VCCs on each node on the end-to-end path and we represent this traffic in figure 2 as the 'External traffic flow'. Since the external traffic occupies the same

1We mean here the models on the 'cell' level (excluding the Adaptation layer functions, which will be described separately) 
buffers as the VC traffic, external and VC-cells may be interleaved. This affects the delay performance of the observed VC-traffic and leads to a more realistic end-to-end delay model. We assume that the external traffic does not continue on the same path as the user traffic and therefore, the it is modeled as a 'cross traffic', as shown in figure 2 .

In the end-to-end delay measures we neglect the delay relevant to the virtual circuit establishment. We also neglect any other delay overhead resulting from the network control or management plane functions.

\section{Two priority classes:}

Our intention here is to distinguish between traffic with stringent time constraints, like voice-traffic, from traffic with no time constraints, like data-base file transfer. We have separate buffers for the two traffic classes. Time-constrained traffic has higher priority at each server on the end-toend path; only if the time-constrained cell queue (voice/video queue in figure 2) is empty, the cells from the data queue will be served.

For simplicity we refer to high priority traffic as 'voice' traffic and to low priority traffic as 'data' traffic.

\section{Network Buffers:}

Due to the finite buffer capacity constraints in the real networks, we modeled the network buffers as finite. As mentioned above, the buffers for the 'high' and 'low' priority traffic are separate, as shown in fig. 4.

We want to point out that the majority of our results is derived for the case of having infinite buffers and no-priority VC-traffic. The reason for this is explained in section 5.1.2.

\section{Propagation Delay:}

The network propagation delay is modeled as an infinite server queue. We have assumed that the individual networks are $100 \mathrm{~km}$ long and, therefore, the propagation delay turns out to be $0.5 \mathrm{~ms} /$ network (the propagation speed is assumed to be $200,000 \mathrm{~km} / \mathrm{s}$ ). Therefore, in the end-to-end delay of our three-hop networks, $1.5 \mathrm{~ms}$ are due to the propagation delay. 
Service Time:

The service time in ATM model is deterministic, and equal to the time of transmitting one ATM cell ${ }^{2}$. In the Megacell network, the service time is general since the packet length distribution is general. The discussion of the cell sizes and the related overhead in both network models is given in the following section.

Before we continue describing the Segmentation, Reassembly and Assembly modules, we need to give a brief overview of the cell sizes and the overhead in the ATM and Megacell network.

\subsection{Cell Formats in ATM and Megacell network}

\section{Megacells:}

In general, VLC networks have some maximum cell-size limit. If the user packet size exceeds the maximum cell-size limit, it is segmented into cells of maximum cell size and the rest of the packet, which can be of any size smaller than the maximum cell size, is transmitted as a cell of a variable length. However, if the cell-size limit in the VLC-type network is set to be very big (e.g. 2 kbytes or 4 kbytes or even bigger [7]) the packet segmentation occurs only for extremely large packets. Therefore, in our models we can assume that the segmentation does not occur at all ${ }^{3}$. The user packets are transmitted as a large variable-size cells in their original size including the megacell header.

\section{Megacell header:}

The header in the Megacell network contains routing information for all the intermediate nodes on the end-to-end path. At each node, routing information for the last hop is stripped-off, so the header gets smaller. Hence, the length of the header is dependent on the number of hops which are left for the particular 'Megacell' to reach the destination (source routing technique, mentioned in [7], for the IBM 'PARIS' network).

2 The transmission speed is assumed to be $100 \mathrm{Mbps}$

3 Since we have assumed that packets arriving to the VLC network will not be segmented in smaller units our models do not include the adaptation layer for the VLC network. The real VLC network implementation, however, will have an adaptation layer to segment very large user packets into some maximum-size megacells. 
We have assumed that the length of the 'megacell' header is uniformly distributed between 3 and 10 bytes. This header is appended to each user packet at the source Megacell network.

The processing time of adding a megacell header is considered to be negligible.

\section{ATM Cells:}

The ATM cell-size is 53-bytes, as specified in the CCITT standard document [1].

\section{Overhead in ATM Cells:}

The overhead in ATM cells is due to the ATM and the ATM Adaptation layer functions. The size of the ATM layer header is 5 bytes. The Adaptation layer overhead (header and trailer) is 4 bytes in the case of a 'type 3' Adaptation Layer, which is assumed in this simulation study ${ }^{4}$. Therefore, the total overhead in each ATM cell is 9 bytes.

The Adaptation layer functions are modeled as separate modules ('Segmentation' module at source and 'Reassembly' module at destination, as shown in figure 1), and are described in the following section.

4 We have discussed the ATM and the Adaptation layer functions and the related overhead formats in detail in the Appendix A. 


\subsection{Segmentation module}

In an ATM network user packets are transported on an integral number of cells and the padding bits are used to complete the unfilled cells if the length of the user packet is not equal to the exact multiple of 44 bytes. Hence, in all topologies where user packets arrive to an ATM-type network, we include a queueing model for the adaptation layer which performs the segmentation of user packets into 44-byte units to fit into the information field of the ATM cells. The processing time for this segmentation is assumed per packet (see the 'adaptation time' in the outline of assumptions, section 5.1.3).

The nine bytes header is added to each cell (four for the adaptation layer and five for the ATM layer). The processing time for adding the cell headers at both layers is included in the 'adaptation time'.

\subsection{Reassembly module}

In all topologies where ATM cells reach the destination network, we include another queueing model for the adaptation layer in which ATM cell headers are stripped-off and cells reassembled into packets and delivered to the user layer.

This module performs also the error checking function and issues retransmission requests for the uncompletely received packets in the case that cells get lost. See section 2.6 for details about modeling of packet retransmissions.

\subsection{Assembly module (topology 3)}

The 'Assembly' module is inserted between the ATM and the Megacell network. Since Megacell network supports variable cell sizes, there is a possibility to assemble a number of ATM cells into a single larger unit and thereby reduce the amount of 'megacell header' information which is appended to each packet entering the Megacell network. Due to transmitting less overhead for the ATM cells with this method, the efficiency of the Megacell network will increase. Moreover, the average end-to-end delay for the user packets can be minimized. 
The function of the 'Assembly' module is, therefore, to buffer the incoming ATM cells, assemble them at a certain point into a single megacell, append a Megacell header and transmit to the Megacell buffer.

Several schemes for assembling ATM cells can be proposed. We will concentrate on the following three schemes:

- A 'Fixed Time-Slice' scheme: Assemble ATM cells every fixed amount of time.

- $\quad$ A 'Fixed Block-Size' scheme: Assemble ATM cells as soon as certain number of ATM cells (block-size) has been accumulated.

- A 'Hybrid' scheme, which is a combination of the first two schemes: Assemble ATM cells every fixed amount of time or as soon as a certain number of cells has been accumulated, whichever event occurs first.

\subsection{Modeling of Packet Retransmissions}

Due to the finite buffers in the networks, cells may get lost. In all the topologies, in the case of a cell loss, the entire packet is retransmitted. The retransmission is on a end-to-end basis, as follows:

If an ATM network is the network to which the destination station is attached to, the destination adaptation layer (in our models the Reassembly block) will request the packet retransmission. Similarly, if the destination station is attached to the Megacell network, as soon as the loss is detected, the retransmission request packet will be issued 5 .

We have assumed that the retransmission request packets are transmitted back to the source as information units of the highest priority. This means that, in our models, the retransmission requests are transmitted back to the source network at each intermediate node without queueing delay.

Since retransmission request packets are relatively small, we have assumed the transmission time for these packets to be negligible. However, since our

5 Notice, in the case of a Megacell network we implemented the function of error checking and requesting packet retransmission as the part of a Megacell network since we do not model the adaptation layer for the Megacell network explicitly, although in reality this function should probably be implemented in the Megacell adaptation layer 
topologies consist of a three-hop networks, it will take three times the network propagation delay for the retransmission-request packets to reach to the source. Therefore, if the retransmissions are frequent, the propagation delay of the retransmission request packets will have considerable impact on the mean end-to-end packet delay.

Having received the retransmission request, the source retransmits the lost packet. In the case that the source is attached to an ATM network, we have modeled the retransmission to occur as soon as the adaptation server finishes the service of the current packet. Similarly, in the case of a source being attached to a Megacell network, upon receiving the request, the source immediately retransmits the requested packet (here megacell) after the service (transmission) of the current megacell has been completed. 


\section{TRAFFIC MODELS}

The traffic in BISDN network is going to be very complex and, consequently, very difficult to model. The complexity arises from the variety of traffic types transmitted on the common network. We have also pointed out that different types of traffic e.g. voice, video and data have different transmission requirements in terms of acceptable delay, cell loss probability etc.

The crucial point is that the broadband network traffic may be very bursty and the inter-arrival times may be highly correlated. Modeling of broadband traffic by using the Poisson assumption is inappropriate and leads to false conclusions concerning the mean end-to-end packet delay and the cell loss probability. Our simulation results clearly demonstrate the major impact of burstiness of the network traffic on the end-to-end delay and the buffer space requirements.

We believe that modeling of bursty traffic is essential and discuss this issue in more detail in this chapter.

\subsection{Burstiness in Broadband Networks}

Let us explain in more detail the cause for the burstiness in broadband networks. Following that, we will discuss the method we chose to model burstiness in this study.

The reason for the bursty network traffic is twofold:

\section{- Arrivals of the user packets:}

User packets may arrive in bursts at the Adaptation Layer. This occurs in the case where the sources are bursty. Examples for the bursty sources would be client/server applications, packetized voice, and variable-rate compressed video. Also, if the traffic which arrives to this network is already multiplexed at some previous point outside the ATM or Megacell network and is composed of various types of bursty and non-bursty sources, the resulting incoming traffic is going to be bursty.

Therefore, regardless of whether we are considering an ATM or a Megacell network, the incoming user traffic may be bursty itself. 
In the case of an ATM network, the Adaptation Layer is going to segment incoming user packets into 53-byte cells and send those in a very short time-intervals (bursts) or perhaps instantaneously to the ATM layer (batched arrivals).

Hence, in ATM, even if the user traffic is not initially bursty, the segmentation process at the adaptation layer is going to generate bursty arrivals at the ATM layer.

\subsection{Superposition of the Arrival Processes}

If the arrivals to the network result from multiple sources and they are multiplexed at the Adaptation layer, the aggregate arrival process, as seen by the ATM layer, is a superposition of the individual arrival processes. In order to avoid modeling each individual arrival source separately, which would lead to huge and impractical models, it is desirable to obtain the characteristics for the aggregate (superposition) arrival process. This leads to simpler models and facilitates the simulation and analytical analysis.

The notion of superpositioning of the individual arrival processes is particularly important in the analytical analysis ${ }^{6}$. For the simulation purposes, we are only interested in modeling the arrival process at the entry points to our network models, i.e. in our case, modeling of the VCand external traffic arrivals. However, we are interested in selecting an arrival process which is also analytically tractable and thereby make the comparison of the simulation and analytical results possible. Since in our example the external traffic consists of multiple superimposed arrival streams, the decision has to be made of how to characterize this superposition arrival process. We discuss the model used to characterize the arrival process of the VC- and external traffic in section 3.5.

In general, it is difficult to characterize the superposition arrival process due to the fact that the successive inter-arrival times of the superposition processes may be correlated.

6 From the analytical point of view, to obtain the end-to-end delay performance one needs to characterize the arrival processes at each individual network component on the end-toend path (e.g. Adaptation and ATM layer at source, ATM layers at intermediate networks etc.). Therefore, in the scope of the analytical models it is of great importance to understand the problems of superpositioning of the arrival streams and characterizing the network departure processes. 
There have been a number of studies on the superposition of arrival processes. In [16] [17] [12] [18] [19], and [20] the inter-arrival time of the superposition process is characterized by its exact mean and an estimate of its coefficient of variation. More recently, Sriram and Whitt [21] studied an aggregate arrival process resulting from superposing multiple bursty sources. Heffes and Lucantoni [22] suggest using a Markov Modulated Poisson Process (MMPP) to characterize a superposition process consisting of multiple voice streams (see section 3.4 and 3.6). Perros and Onvural [23] obtained an exact pdf of a single interval of the superposition of Interrupted Poisson processes. An alternative approach to studying a single queue with $\mathrm{N}$ different arrivals is to use the fluid-flow approximations technique, e.g. [24]. Various models for analyzing single queue with $\mathbf{N}$ voice arrivals are discussed in [25].

A more detailed overview of the literature on the superposition of arrival processes is given in Perros [26]. The author emphasizes the need for further research in this area, in particular to obtain simple approximate expressions which will have good accuracy and can be incorporated in larger approximate models.

\subsection{Validity of a Poisson Process in Broadband Networks}

The Poisson arrival process has proven to be very successful in modeling arrivals of non-bursty sources, to a broadcast network or to a telephone switch. The Poisson model assumes that the inter-arrival times are independent, exponentially distributed random variables.

In the case of a complex broadband traffic the situation is quite different, especially in ATM networks. Since the user packets may arrive in bursts and the packets are being segmented into cells, the arrivals to the ATM and, in-turn, Physical Layer occur in bursts. The inter-arrival times of bursty arrivals are correlated. Therefore, the Poisson process does not model satisfactory the user arrival process.

If the individual arrivals to the network are non-Poisson, the traffic on the communication links is also not going to be Poisson. This is obvious, but the interesting question is whether we can approximate the traffic on the communication links with a Poisson process since the communication links carry an aggregate network traffic. The Poisson assumption would considerably facilitate the analytical models, which will be undertaken in our future work. 
During the course of this simulation project we have made several experiments to evaluate the burstiness of the network inter-arrival and inter-departure times for various network arrival processes and packet length distributions. In general, one could speculate that the superposition of the two arrival processes, in our model the VC-traffic and the external traffic, leads to a 'smoother' aggregate traffic, on average. We have found that the burstiness of the $\mathrm{VC}$ - departure process as well as the aggregate departure process (composed of VC- and external traffic) may get worse (more bursty) even in the case where the bursty VC-traffic is multiplexed with Poisson external arrivals. We also found that the results are greatly influenced by the packet length distributions of the incoming traffic. The results of this research are shown in the Appendix B.

From the above discussion it becomes evident that the Poisson arrival process can be used in our models only at the entry point to our networks if we assume that the user traffic (VC-traffic) is a 'smooth' traffic.

We have used the Poisson assumption for the VC- and External traffic in the case of Megacell networks in our initial runs basically for simplicity reasons for the simulations ( see section 5.1.3 for more details). In the simulation runs for the ATM network, we have used Poisson arrivals for the VC-traffic and assumed the batch Poisson process for the external traffic arrivals.

The authors in the papers that we mentioned in the literature survey (section 1.4) used only the Poisson assumption to model the arrival process of the user packets to the network. Because of the bursty traffic arrivals and correlated inter-arrival times in broadband networks, it is evident that the comparison of the ATM and Variable Length Cell-size networks by using the Poisson assumption for the arrival processes needs to be extended to an arrival process which is more appropriate for the broadband environment. 


\subsection{Markov Modulated Poisson Process (MMPP)}

The two-state Markov Modulated Poisson Process is a doubly stochastic process in which the arrival rate depends on the state of the underlying Markov chain. The duration of time a system is in a certain state is exponentially distributed with parameters $1 / \alpha$ and $1 / \beta$ respectively for the states 1 and 2 . The inter-arrival times in states 1 and 2 are exponentially distributed with mean arrival rates of $\lambda_{1}$ and $\lambda_{2}$. A two-state MMPP is shown in figure 3.

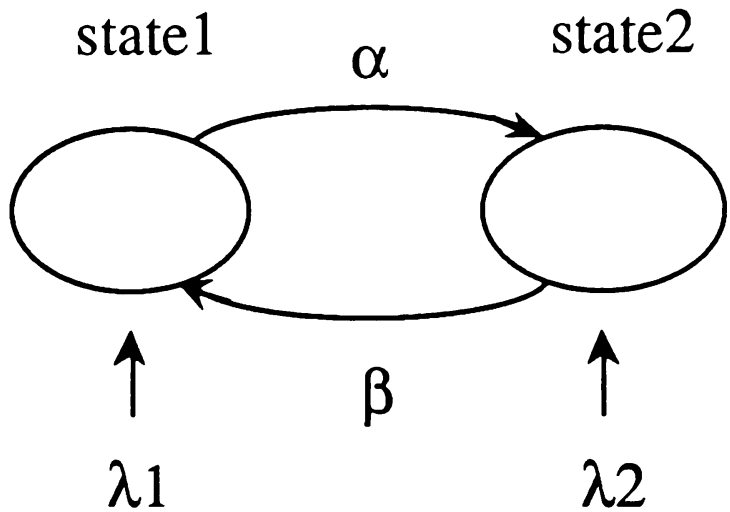

figure 3. Two-state Markov Modulated Poisson Process

Let us assume that the arrival rate in state 1 is considerably higher than the arrival rate in state 2 . Thus, state 1 can represent a burst of the arrivals with the duration of the burst $1 / \alpha$. The burst is followed by a period of a low arrival rate of the length $1 / \beta$, on average. In this way one can address the problem of generating bursty arrivals.

Another issue is the correlation of the inter-arrival times in an MMPP. The experiments demonstrate that MMPP generates a sequence of inter-arrival times which are correlated. Thus, MMPP can capture the correlation of inter-arrival times, which is expected in broadband network traffic. However, the analytical expression for the correlation of inter-arrival times in MMPP is very difficult to obtain. Without an analytical expression we are unable to define the combination of the values for the MMPP parameters to achieve the desired values for the correlation of inter-arrival times. Obtaining an analytical solution to the correlation of the MMPP inter-arrival times is a very important issue and requires further investigation. 


\subsection{Modeling the VC- and External Traffic-Arrivals with Markov Modulated Poisson Process}

Since the user packets (VC-traffic packets) may arrive to the network virtually at any time, and the arrivals may be bursty, the MMPP is a good model for both the ATM and the Megacell network.

Considering the external network arrivals, the situation is more complicated. The problem arises because, as indicated earlier, this traffic is assumed to have traversed several network nodes before interfering with our VC-traffic. Moreover, the external traffic is modeled at the ATM layer in the intermediate network nodes, as opposed to the user traffic which is modeled as an incoming traffic to the Adaptation layer. Therefore, the external network arrivals need to be modeled differently in the case of ATM and Megacell networks.

In the case of a Megacell network, since there is no segmentation of user packets and packet lengths are variable, the arrival process of the packets from the external traffic is going to be similar to the arrival process of the VC-traffic and can be modeled with an MMPP process.

However, in the case of an ATM network, due to the fixed cell sizes, the inter-arrival times of the external traffic are going to occur in a discrete fashion. Therefore, in the case of the external arrivals to the ATM networks, a discrete arrival process seems to be more appropriate. A discrete-time counterpart to the MMPP is the Markov Modulated Bernoulli Process $(M M B P)$.

In the MMBP, the assumption is that the arrivals occur only at regular time-instances (beginning of slots) and the packet lengths are geometrically distributed. In the two-state MMBP, we can distinguish period with high and low arrival rates. In the 'high' arrival rate state, there is a high probability, p, of having an arrival (cell) in the next slot, and a low probability, 1-p, of having the next slot idle. Correspondingly, in a 'low' arrival state, there is a high probability of having no cell in the next slot, $q$, and a low probability, 1-q, of having a cell in the next slot.

Intuitively, an ATM network appears as a slotted system, where the interarrival and inter-departure times occur at slot boundaries. We could postulate that the MMBP may be a good model for the external traffic to the ATM networks. However, if we assume that the cells are forwarded in the switches as soon as the outcoming port becomes available, which is a feasible implementation option in ATM switching, then the notion of 
having a strictly deterministic system, where everything occurs at slot boundaries, is no longer valid.

This is particularly true if we assume that the adaptation layer is going to be implemented in a highly parallel architecture, so it can process several incoming packets simultaneously and send the corresponding cells immediately to the buffers of the ATM layer (or pass pointers to the buffers in the case of shared memory ATM switches). Moreover, if we assume that the external traffic which arrives to our networks is an aggregated traffic from multiple sources and the switches do not operate in a strict 'sloted-fashion', then, it is justified to use an MMPP process to model the external arrivals not only for the Megacell network but for the ATM network as well.

\subsection{Characterization of Burstiness of the Arrival Process}

The notion of burstiness in broadband networks is undisputed. The question remains how does one quantify burstiness?

There have been several different approaches in the literature. In [22] Heffes and Lucantoni use the ratio of the variance to the mean of the number of arrivals in the interval $(0, \mathrm{t})$ :

$\frac{\operatorname{Var}\left(N_{t}\right)}{E\left(N_{t}\right)}=1+\frac{2\left(\lambda_{1}-\lambda_{2}\right)^{2} \alpha \beta}{(\alpha+\beta)^{2}\left(\lambda_{1} \beta+\lambda_{2} \alpha\right)}-\frac{2\left(\lambda_{1}-\lambda_{2}\right)^{3} \alpha \beta}{(\alpha+\beta)^{2}\left(\lambda_{1} \beta+\lambda_{2} \alpha\right)} \frac{1-\mathrm{e}(\alpha+\beta)_{t}}{t} \quad / 1 /$

To characterize an aggregate packet arrival process, resulting from the superposition of voice and data streams, Sriram and Whitt [21] use the Index of Dispersion per Intervals (IDI), based on results from Cox [9] and Whitt [17]. The IDI, also called the $k$-interval squared coefficient of variation sequence, describes cumulative covariance among successive inter-arrival times:

$c_{k}{ }^{2}=\frac{k \operatorname{Var}\left(S_{k}\right)}{\left[E\left(S_{k}\right)\right]^{2}}=\frac{\operatorname{Var}\left(S_{k}\right)}{k\left[E\left(X_{1}\right)\right]^{2}}=c_{1}{ }^{2}+\frac{\sum_{i, j=1}^{k} \operatorname{cov}\left(X_{i}, X_{j}\right)}{k\left[E\left(X_{1}\right)\right]^{2}}$ 
where $S_{k}$ is the sum of $k$ consecutive inter-arrival times $X_{i}$ and the $\operatorname{cov}\left(X_{i}\right.$, $\left.X_{j}\right)=E\left(X_{i} X_{j}\right)-E\left(X_{i}\right) E\left(X_{j}\right)$.

We prefer to use the squared coefficient of variation of inter-arrival time, $c^{2}$. The squared coefficient of variation of inter-arrival time is defined as the variance over the mean square of the inter-arrival times.

$c^{2}=1+\frac{2 \alpha \beta\left(\lambda_{1}-\lambda_{2}\right)^{2}}{\left(\beta \lambda_{1}+\alpha \lambda_{2}+\lambda_{1} \lambda_{2}\right)(\alpha+\beta)^{2}}$

We obtained the $c^{2}$ of the inter-arrival time after deriving the pdf of interarrival time for a MMPP. The derivation of the MMPP pdf of inter-arrival times and squared coefficient of variation of inter-arrival times is given in Appendix C.

As an illustration of the use of $c^{2}$ to characterize 'burstiness' of an arrival process, we show an example where we assume that the mean time until state change in states 1 and $2,1 / \alpha$ and $1 / \beta$, are equal to 1 . Let us also assume that the arrival rate from state 1 is constant, equal to 1 . We vary the arrival rate from state 2 in the range 1 to 100 . The burstiness $\left(c^{2}\right)$ of the MMPP process increases as the arrival rate from state 2 increases, as shown in figure 4.

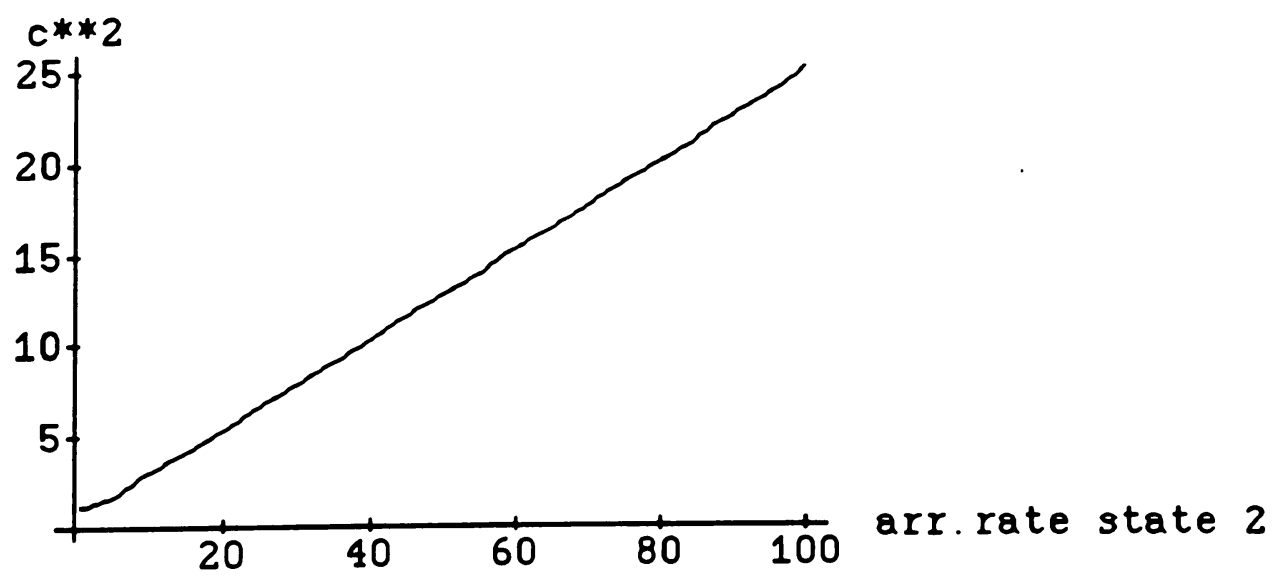

figure 4. Burstiness $\left(c^{2}\right)$ vs. Arrival rate in state $2\left(\lambda_{2}\right)$

Notice that the $c^{2}$ curve starts out with the value 1 in the above example. Since initially the mean arrival rates in the two states are equal $\left(\lambda_{1}=\lambda_{2}=1\right)$, 
the MMPP is in this case is reduced to a single Poisson processes. Hence, $c^{2}$ is equal to 1 .

\subsection{Packet Length Distributions}

In our models we have adopted the packet length distribution used in [6] for the comparison of the VLC and ATM network performance. The distribution is shown in figure 5. This packet length distribution is not based on particular packet length measurements. However, if we compare this distribution with the measurements stated in [13] [14] [15], it provides a reasonable average situation.

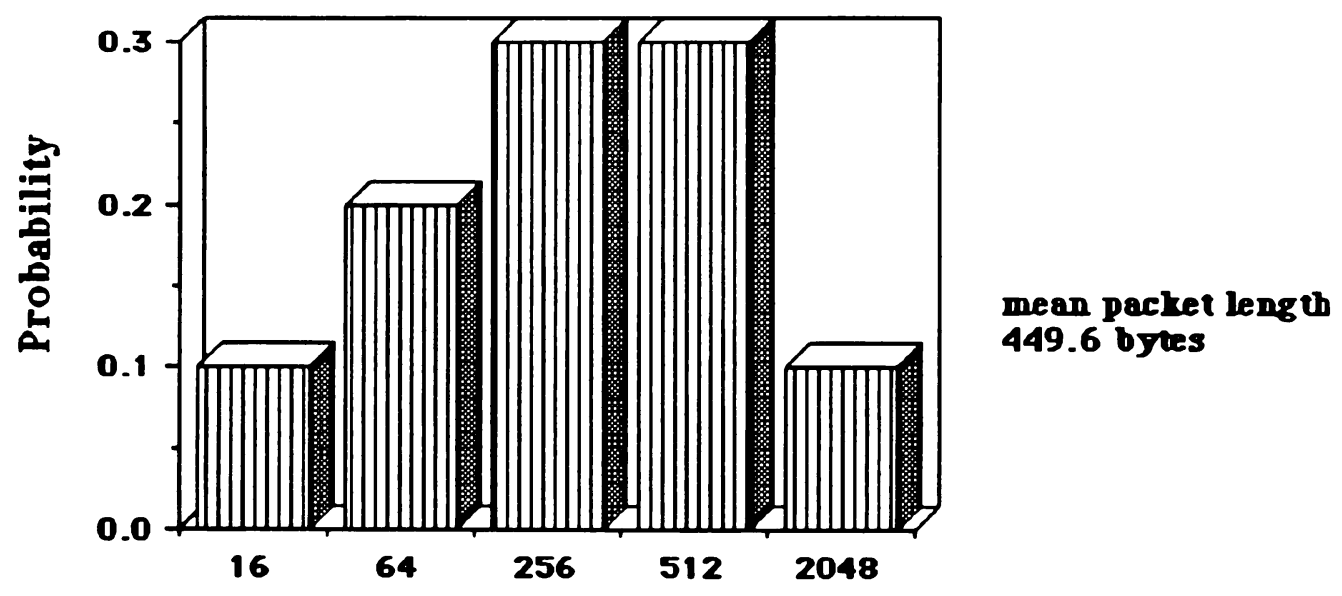

Packet Length [bytes]

figure 5. Packet Length Distribution?

We have also done experiments with other packet length distributions, particularly for the sake of comparing the end-to-end performance for topologies 1 and 2 with respect to the submitted packet lengths. The results include studies for packet lengths of 480 bytes and 4500 bytes. The 480byte packets are represented in the ATM network with 10 ATM cells and no padding bits (assuming in this example that no header is added by the ATM Adaptation Layer). The experiments in which we assumed that the incoming packets are 4500 bytes (maximum packet size in the FDDI network), are designed to evaluate the end-to-end delay for the bulk data transfers.

7 We refer to this distribution as a 'variable' packet length distribution later in the report 


\section{SIMULATION TOOL}

\section{QNAP2}

The simulation programs were written in QNAP2 (Queueing Network Analysis Package) [27]. The first version of the program was written by INRIA in France in 1978 and since then it has constantly been upgraded and used by numerous research groups. QNAP2 has been used at CCSP for the last five years.

The modeling framework supported by QNAP2 is the representation of a system as a network of stations through which circulate customers (a station being defined as one or several servers serving a single input queue). The stations represent physical or logical processing facilities of the system to be modeled, and the customers represent the processes being executed and competing for these facilities. For each station, the actions required to handle the particular customer (time delays, synchronization operations, customer generation, ...) must be specified as well as the corresponding routing rules. These actions are executed by one of the servers of the station, according to the station scheduling policy (FIFO, priority, ...). The language features allow building any complex mechanism.

The modeling framework of QNAP2 makes it possible to support a large set of analysis techniques developed for standard or generalized queueing network models. QNAP2 implements analytical solvers (convolution algorithms, mean value analysis and approximate methods for productform networks), discrete event simulation, and a Markovian solver of specific interest for the exact analysis of limited size models involving intricate mechanisms. 


\section{DISCUSSION OF RESULTS}

\subsection{Introduction}

Prior to the discussion of results, we address briefly the complexity of the simulation models and explain the reason for restricting the results to a single class of traffic. Following, we give an outline of the assumptions and parameters that were used in the study.

A detailed block diagram for the models of each topology is presented. The assumptions used in the individual studies are described along with the discussion of the simulation results. Details concerning the models of individual network components are described in Section 2.

\subsubsection{The focus of the study}

In all simulation studies we have focused on the end-to-end delay for data packets of the VC-traffic (user traffic). Particular attention was given to modeling of bursty arrivals and obtaining end-to-end results for different burstiness and packet length distribution. Since evaluating the memory requirements at the intermediate network buffers is also a very important issue, we have provided results reflecting the mean and maximum buffer size requirements for the majority of simulation runs.

\subsubsection{The choice of the assumptions for the studies}

In the simulation programs for topologies 1-4 we have implemented all the assumptions which are described in chapters 2 and 3, including finite buffers, bursty arrivals and two classes of traffic. Since end-to-end topology models are fairly complex and the execution times for the simulation runs are long, especially in the case of interconnected heterogeneous networks, having all the assumptions simultaneously in the model would diminish our capability of making conclusions about the impact of the individual parameters on the end-to-end delay. Hence, depending on the desired comparisons, we have made restrictions as to what assumption we would like to keep in the model for a particular simulation run. Thereby, we reduced the CPU-time per simulation run and obtained more runs for different assumptions.

We felt that it was very important to obtain end-to-end delay results for the case of bursty arrivals since the end-to-end delay is very much influenced 
by the burstiness of the arrival process. Therefore, we have decided early in the project to obtain results for one class of traffic only and concentrate our efforts more toward evaluating the end-to-end delay performance for different arrival processes. In particular, we focused on obtaining delay results for bursty arrivals using MMPP and IPP arrival processes.

\subsubsection{Outline of Assumptions for Model Parameters}

\begin{tabular}{|c|c|}
\hline $\begin{array}{l}\text { Arrival process for the } \\
\text { Virtual Circuit traffic } \\
\end{array}$ & Poisson, MMPP, IPP \\
\hline $\begin{array}{l}\text { Arrival process for the } \\
\text { External traffic } \\
\end{array}$ & $\begin{array}{l}\text { Poisson, MMPP } \\
(20,50 \mathrm{Mb} / \mathrm{s}) \\
\end{array}$ \\
\hline \multirow{3}{*}{ Packet length distribution } & $\begin{array}{l}\text { variable } \\
\text { (mean } 449.6 \text { bytes) } \\
\end{array}$ \\
\hline & constant ( 480 bytes) \\
\hline & constant $(4500$ bytes $)$ \\
\hline ATM cell size & $\begin{array}{l}53 \text { bytes } \\
(44 \text { bytes information } \\
9 \text { bytes overhead ) }\end{array}$ \\
\hline Megacell size & $\begin{array}{l}\text { equal to packet size }+ \\
3-10 \text { bytes overhead }\end{array}$ \\
\hline Network buffers & infinite 8 (no cell loss) \\
\hline Priority classes & one $^{8} \quad(d a t$ a only $)$ \\
\hline Propagation delay & $0.5 \mathrm{~ms} /$ network \\
\hline Transm. speed of each network & $100 \mathrm{Mbps}^{8}$ \\
\hline Adaptation time & $0.05 \mathrm{~ms} /$ packet \\
\hline Reassembly processing time & $0.004 \mathrm{~ms} /$ cell \\
\hline
\end{tabular}

Table 1. Outline of assumptions for model parameters

8 unless otherwise specified 
The assumptions on the arrival processes and packet length distributions are explained in Chapter 3; 'ATM' and 'Megacell' cell-sizes and overhead formats are described in Chapter 2 and Appendix A; network buffers and propagation delay assumptions are discussed in Chapter 2; traffic priority classes are described in Chapter 2 and Section 5.1. The assumptions concerning network transmission speeds and processing times for the adaptation (segmentation) and reassembly functions were determined in a discussion with IBM.

\section{Calculation of mean arrival rates:}

The end-to-end delays are obtained for the case of fixed arrival rates for the external network arrivals and variable arrival rates for the VC-traffic. In the majority of studies we have assumed external network arrivals to be $20 \mathrm{Mbps}$ and the VC- arrival rates in the range from 10 to $85 \mathrm{Mbps}$, yielding network utilizations of 30 to $95 \%$.

We have calculated the mean arrival rates in the case of a Megacell and ATM network as follows:

In the case of a Megacell network, we have divided network transmission speed by the average number of bytes transmitted per packet (i.e. including average packet length and average Megacell-header length of 6.5 bytes).

Table 2 shows the average overhead (in bytes) transmitted per packet for the 'variable' packet length distribution used in this study in the case of an ATM and Megacell network.

\begin{tabular}{|l|l|l|l|l|}
\hline Probability & $\begin{array}{l}\text { Packet length } \\
\text { [bytes] }\end{array}$ & $\begin{array}{l}\text { \# of ATM cells } \\
\text { needed }\end{array}$ & $\begin{array}{l}\text { Overhead / packet } \\
\text { in ATM [bytes] }\end{array}$ & $\begin{array}{l}\text { Overhead / packet } \\
\text { in Megacell [bytes] }\end{array}$ \\
\hline \hline 0.1 & 16 & 1 & 37 & 6.5 \\
\hline 0.2 & 64 & 2 & 42 & 6.5 \\
\hline 0.3 & 256 & 6 & 62 & 6.5 \\
\hline 0.3 & 512 & 12 & 124 & 6.5 \\
\hline 0.1 & 2048 & 47 & 443 & 6.5 \\
\hline \hline Mean & 449.6 & 10.6 & 112.2 & 6.5 \\
\hline
\end{tabular}

Table 2. 'Variable' packet length distribution and the overhead transmitted in the case of an ATM and Megacell network 
In the case of an ATM network, we first derived the average number of ATM cells needed for transmitting data packets of the particular distribution. Recall that each ATM cell is carrying 44 information bytes (in the case that no padding bits are added for the unfilled cells) and the 9 bytes overhead 9 . The average number of ATM cells is multiplied by 53 bytes, the ATM cell size, which gives the average number of bytes transmitted per packet in ATM network.

Therefore, the mean number of bytes transmitted per packet in the case of the 'variable' packet length distribution is:

- Topology 1 (Megacell networks):

- Topology 2 (ATM networks):

$$
\begin{aligned}
& 449.6+6.5=456.1 \text { bytes } \\
& 10.6 \cdot 53=561.8 \text { bytes }
\end{aligned}
$$

\subsubsection{Plotting of End-to-end Delay Graphs}

Since mean arrival rate is equal to the network throughput in the case of no cell loss (no packet retransmission) in the network, we can use the term 'Network throughput' or 'Network load' instead of the mean arrival rate in the end-to-end delay diagrams.

We present the end-to-end delay in terms of 'User throughput' and 'Network load'. The 'User throughput' is the amount of user information (excl. overhead) transmitted over a unit-time period. The 'Network Load' (due to the VC-traffic) is the total amount of user traffic (VC-traffic) transmitted by the network in a unit-time period (incl. overhead of the ATM or Megacell network). The two curves plotted next to each other enable us to see the effect of the amount of overhead on the end-to-end delay.

\subsubsection{Accuracy of the simulation results}

In the area of network utilizations up to $90 \%$ the relative errors for the results were typically less than $2 \%$ (based upon $95 \%$ confidence intervals). The errors were much lower in the case of Poisson arrival assumptions rather then with the bursy arrivals and, consequently, the simulation runs were shorter.

The results in the region of very high network utilizations (above 90\%) are difficult to simulate with a satisfactory confidence intervals. Typically,

9 Details on overhead in ATM cells are discussed in the Appendix A 
relative errors for the results in the high network utilizations were even more than $10 \%$ for some cases of very bursty input traffic.

Due to a very large number of results we have for simplicity reasons not shown confidence intervals in all the graphs. 


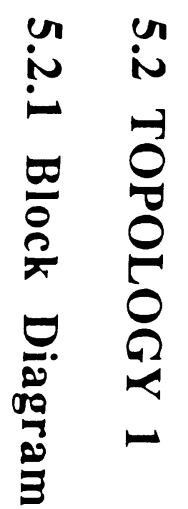

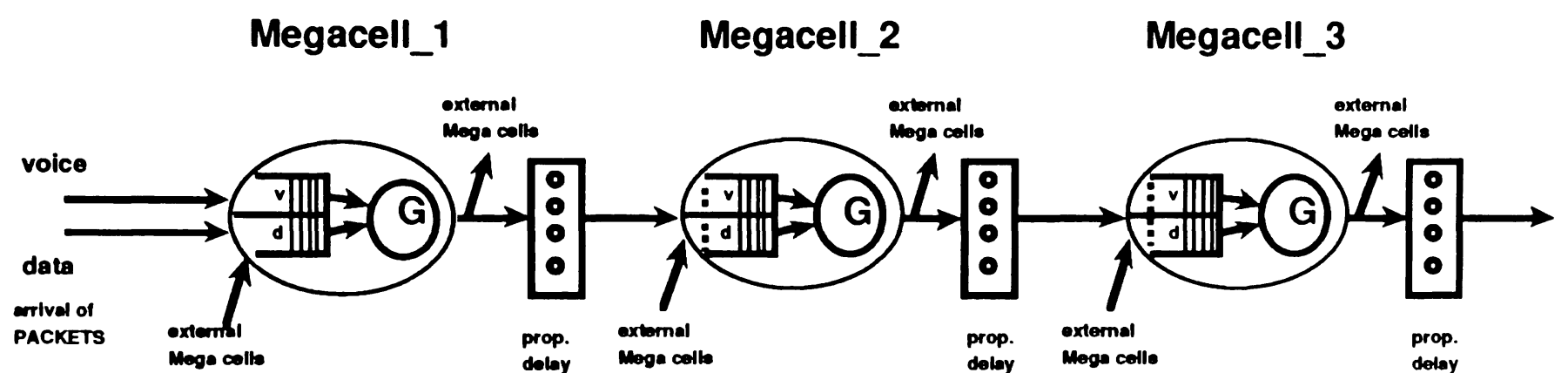


Remark:

In the simulation model, the time for appending Megacell header at the source and stripping the header off at the destination is neglected.

\subsubsection{Discussion of Results}

We start out with the results for the cases in which we assume 'variable'10 packet length distribution for both the VC-and Ext. traffic. External arrivals are $20 \mathrm{Mbps}$ to each network. The arrivals for the VC- and External traffic are varied:

- $\quad$ VC-traffic and External traffic: $\quad$ Poisson

Figure 6 shows the end-to-end packet delay as a function of user throughput and network load due to the VC-traffic.

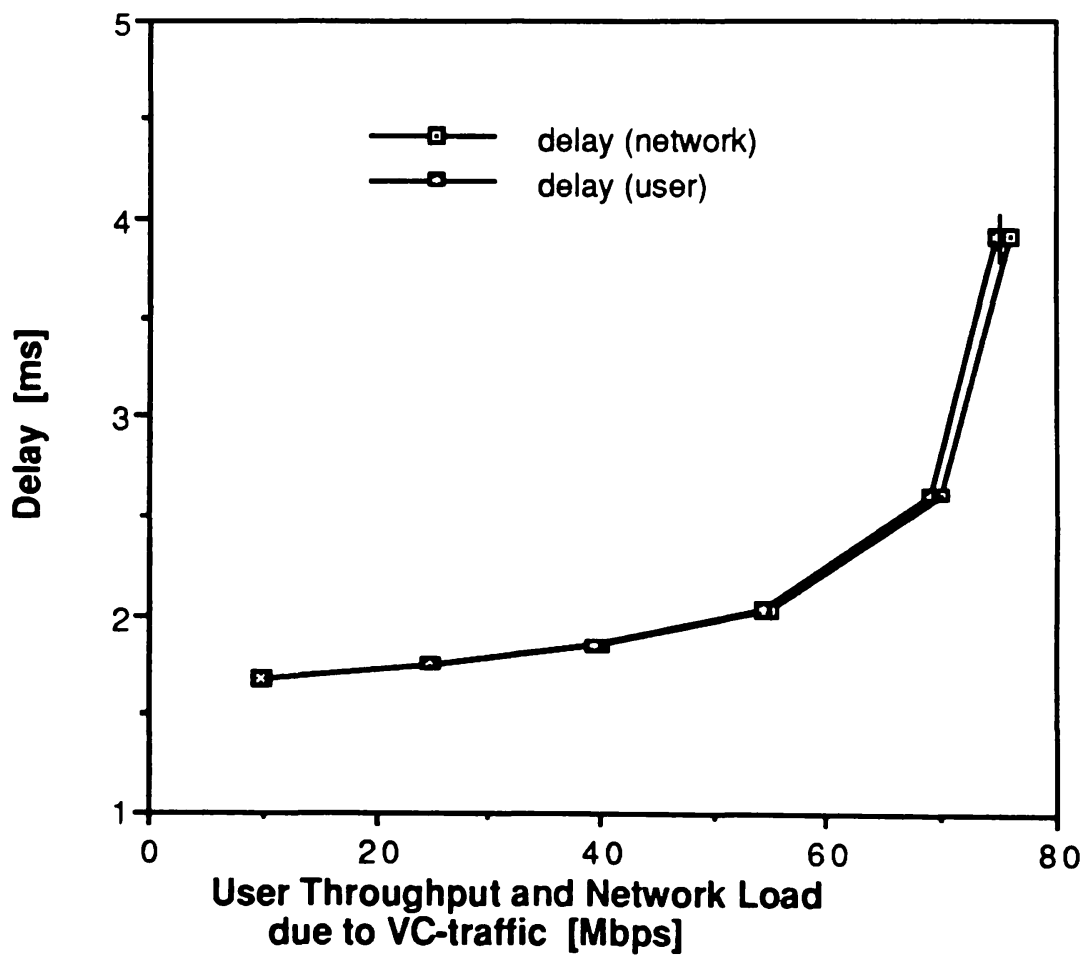

Figure 6. Mean end-to-end delay as a function of user throughput and network load : topology1

10 Distribution of packet sizes is shown in table 2 or section 3.7 , figure 5 
Recall that the overhead in the Megacell network is only 3-10 bytes per megacell, and the packets are not segmented. Since average packet length is 449.6 bytes, and the average overhead is 6.5 bytes, the percentage of overhead in Megacell network is very small $(6.5 / 449.6=1.45 \%)$; consequently, the expansion factor is only 1.0145. Therefore, the curves reflecting the end-to-end packet delay with respect to the network load and the throughput for the user traffic in the case of a Megacell network are very close. The same observation holds in all diagrams involving the Megacell network.

Figure 7 shows average and maximum buffer requirements at each Megacell network as a function of the total network load (VC- and External traffic).

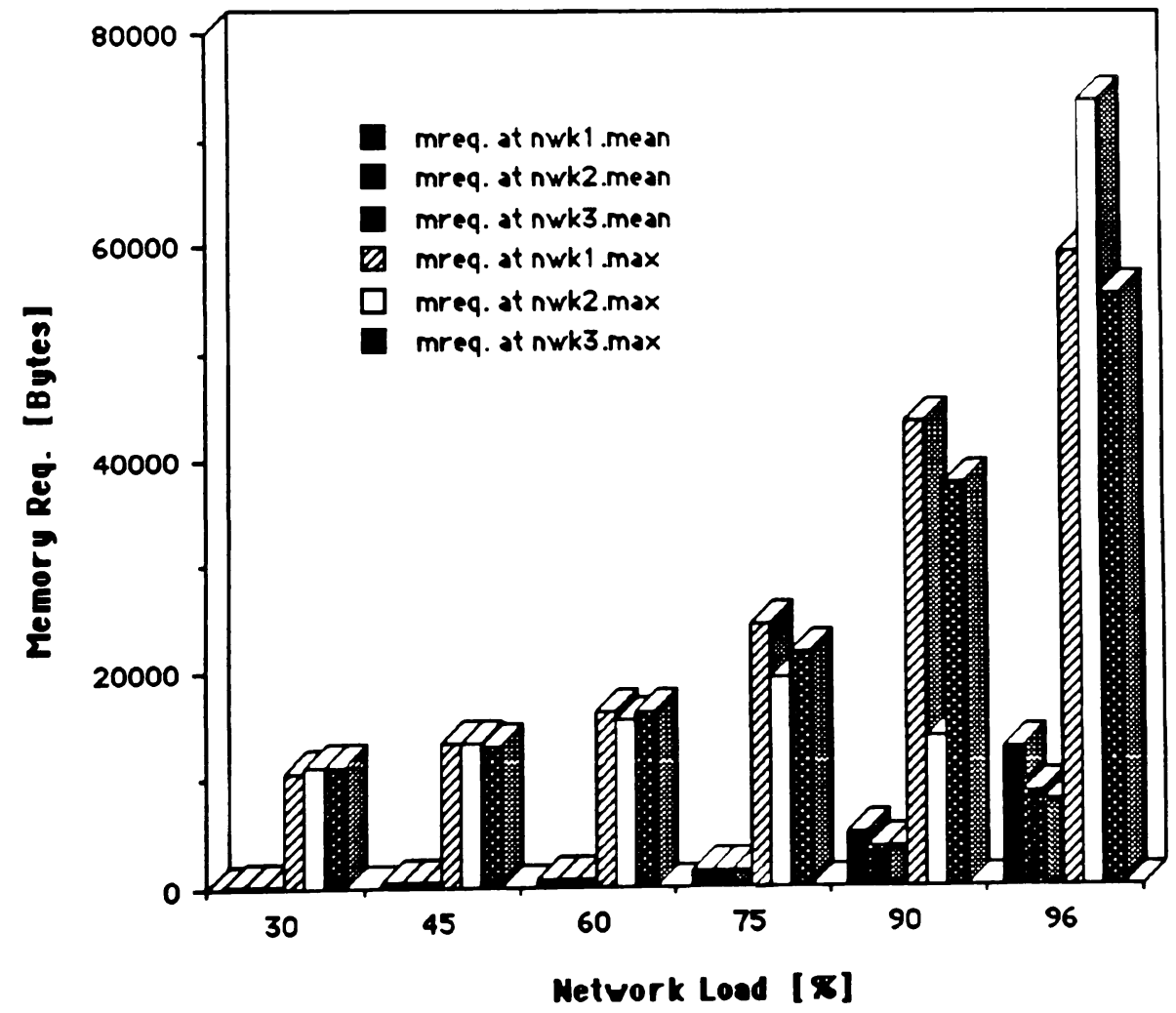

Figure 7. Mean and maximum memory requirements as a function of total network load : topology 1

In figure 8 we plot the end-to-end delay performance for the case of:

- $\quad$ VC-traffic and External traffic: $\quad$ MMPP 


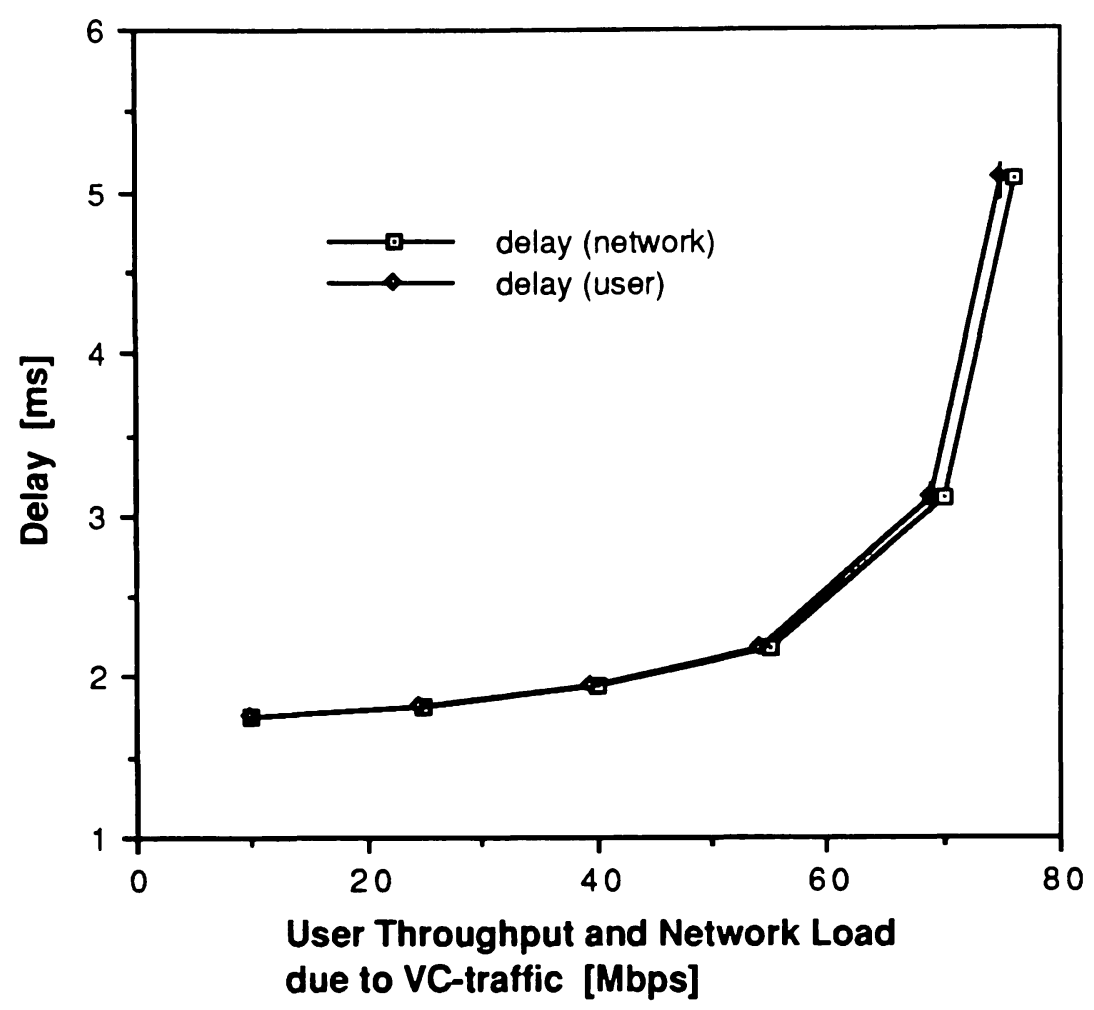

Figure 8. Mean end-to-end delay as a function of user throughput and network load : topology1

In this example the squared coefficient of variation of inter-arrival times $\left(c^{2}\right)$ for the VC-arrivals is chosen to be constant, equal to the $c^{2}$ of the external traffic for all the simulation points in this study. The parameters ${ }^{11}$ for the VC- and External-MMPPs are shown in Table 3.

\begin{tabular}{|l||c|c|c|c|c|}
\hline parameters & $\lambda 1[\mathrm{Mb} / \mathrm{s}]^{12}$ & $\lambda 2[\mathrm{Mb} / \mathrm{s}]$ & $\alpha[1 / \mathrm{ms}]$ & $\beta[1 / \mathrm{ms}]$ & $\mathrm{c}^{2}$ \\
\hline \hline VC-MMPP & 85 & 5 & variable & variable & 1.915 \\
\hline Ext -MMPPs & 80 & 10 & 6 & 1 & 1.915 \\
\hline
\end{tabular}

Table 3. Parameters for the arrival processes (the 'MMPP' case)

By choosing $\lambda_{1}$ and $\lambda_{2}$ to be constant, in order to keep the $c^{2}$ for the VCtraffic constant, the parameters for $\alpha$ and $\beta$ have to be calculated for each

11 For the discussion of MMPP parameters see Section 3.4

12 In the implementation of MMPPs, $\lambda_{1}$ and $\lambda_{2}$ have to be expressed in terms of packets/ms 
VC-mean arrival rate, $\lambda$. The $\alpha$ and $\beta$ are determined from the equations $/ 16 /$ and $/ 17 /$, derived in the Appendix $C$.

Figure 9 compares the end-to-end delay for the Poisson and MMPP arrivals shown in figures 6 and 8 .

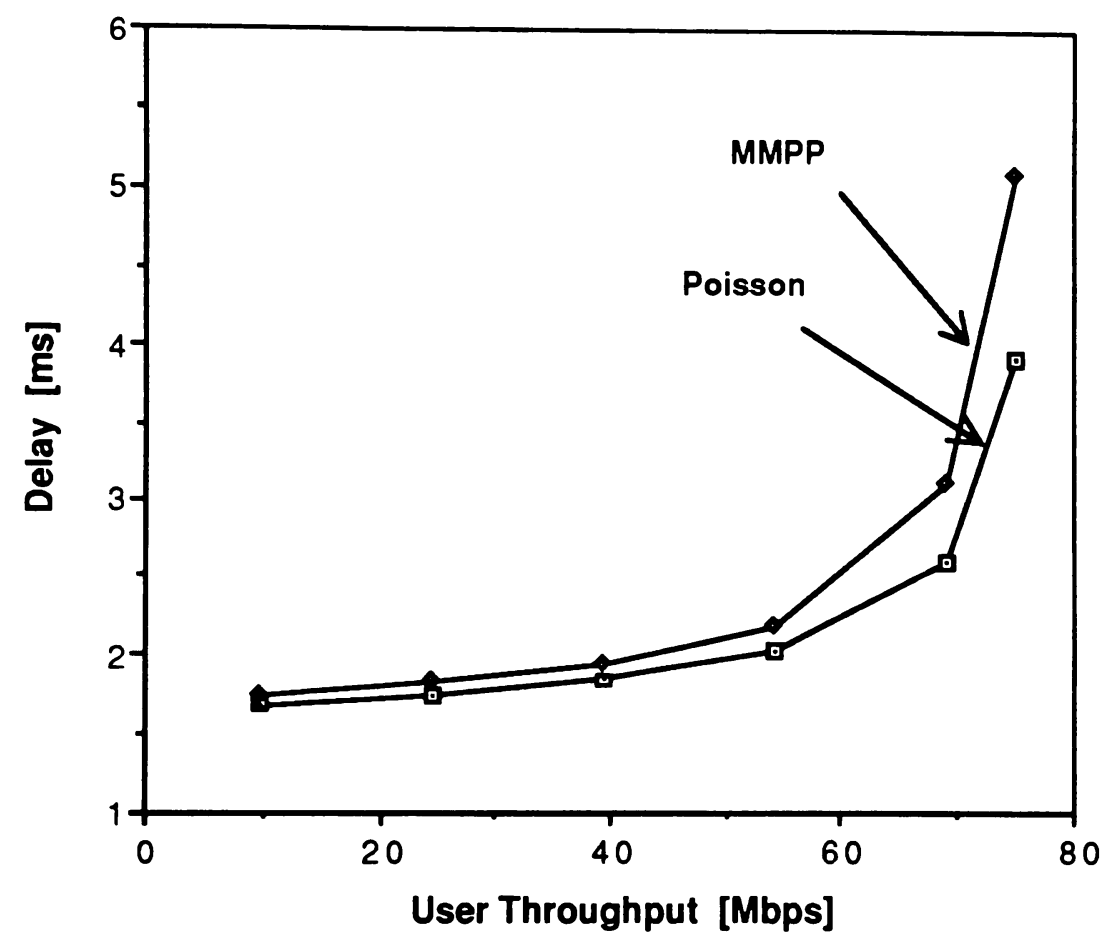

Figure 9. Mean end-to-end delay as a function of user throughput:

Poisson vs. MMPP arrivals, topology1

The conclusion is obvious: Bursty arrivals lead to higher end-to-end packet delay.

In the next two graphs (figure 10,11 ) we compare the mean and maximum buffer requirements for the buffers at networks 1,2 and 3 for the above cases of Poisson and MMPP arrivals. The diagrams show that in the case of bursty arrivals the buffer requirements are significantly higher, particularly in the case of the maximum memory requirements (figure 11). 


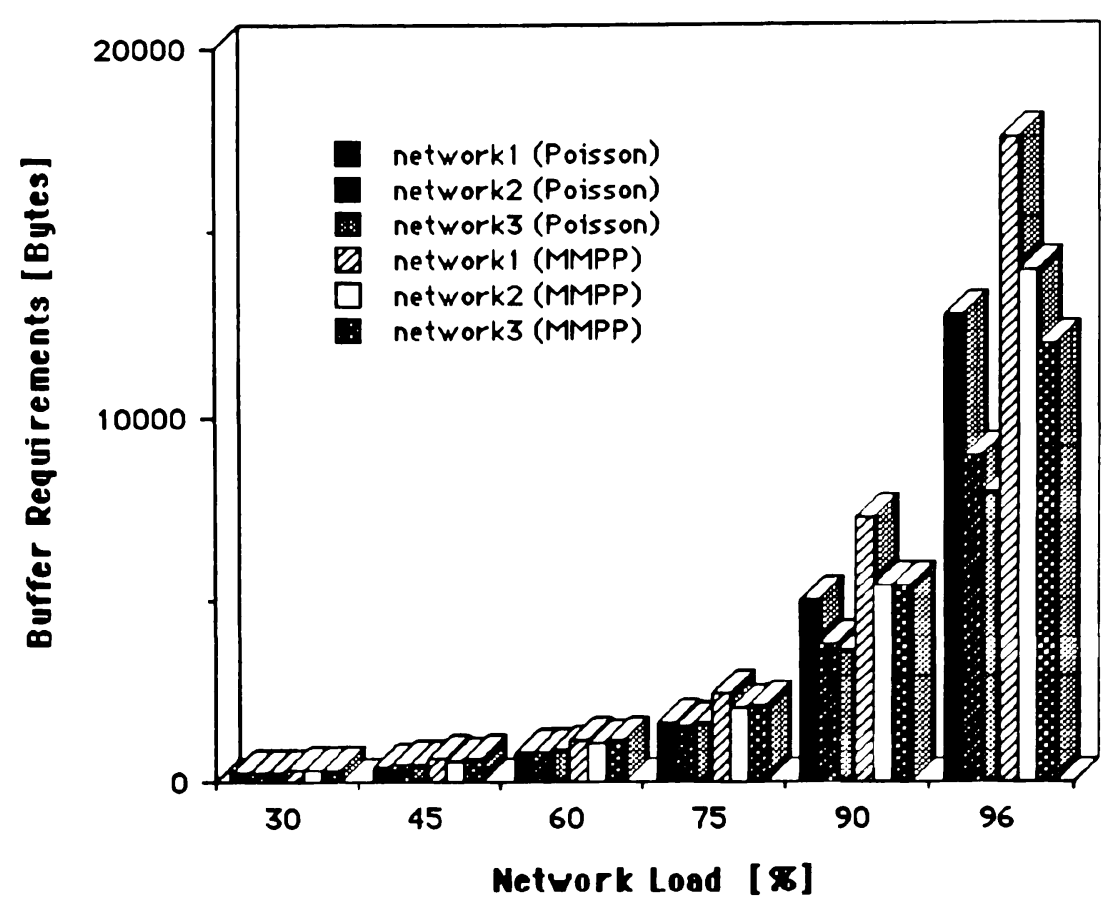

Figure 10. Mean buffer requirements at networks $1,2,3$ as a function of total network load : topology 1

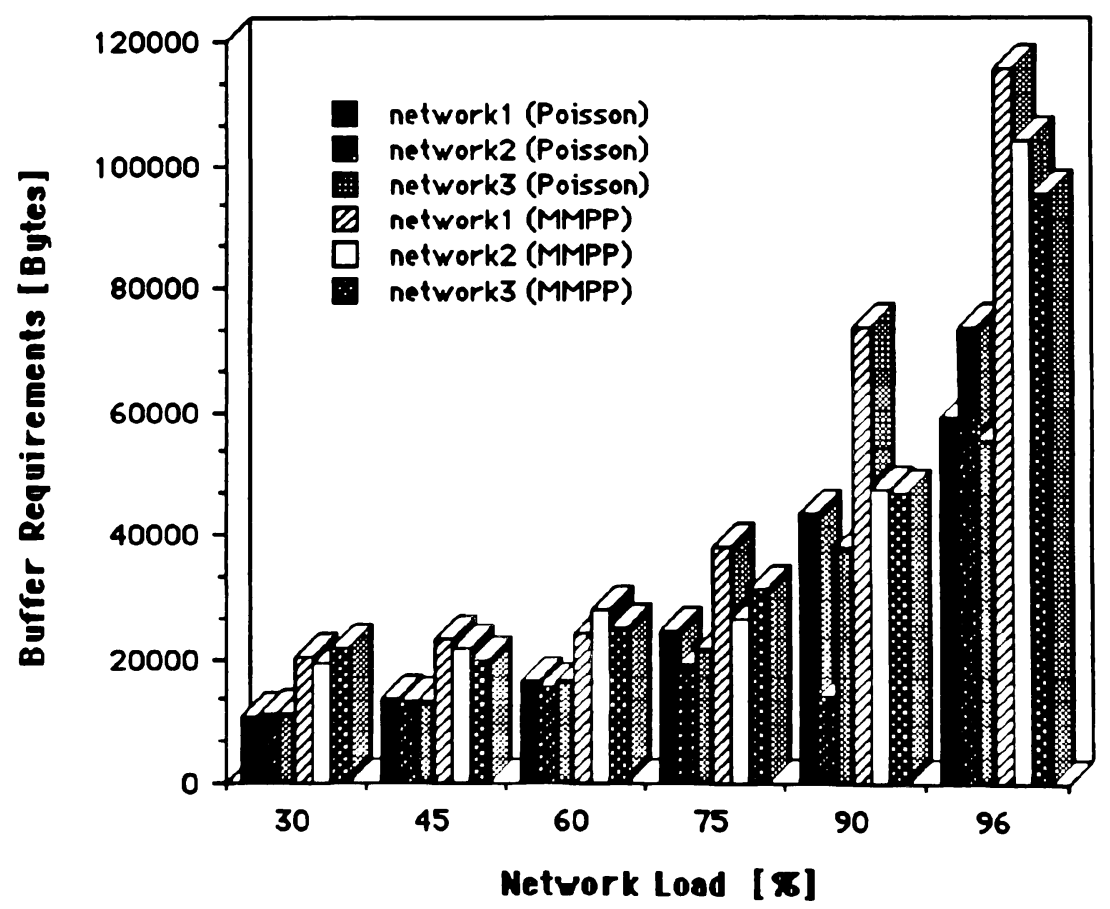

Figure 11. Maximum buffer requirements at networks $1,2,3$ as a function of total network load : topology 1 
- VC-traffic : IPP ${ }^{13} \quad$ External traffic : MMPP

In this study we increase the burstiness of the VC-traffic. VC-arrival process is now IPP and the burstiness $\left(c^{2}\right)$ is 10 . The parameters for the VC-IPP and External-MMPPs used in this example are shown in Table 4.

\begin{tabular}{|l||c|c|c|c|c|}
\hline parameters & $\lambda 1[\mathrm{Mb} / \mathrm{s}]$ & $\lambda 2[\mathrm{Mb} / \mathrm{s}]$ & $\alpha[1 / \mathrm{ms}]$ & $\beta[1 / \mathrm{ms}]$ & $\mathrm{c}^{2}$ \\
\hline \hline VC-IPP & 80 & 0 & variable & variable & 10 \\
\hline Ext -MMPPs & 80 & 10 & 6 & 1 & 1.915 \\
\hline
\end{tabular}

Table 4. Parameters for the arrival processes (the 'IPP' case)

Notice that this study differs from the previous study, where all arrivals were MMPP, only in the burstiness of the VC-arrival process. The packet lengths of the VC- and external traffic and the parameters of the ExternalMMPPs are the same. The mean arrival rates for the external traffic are 20 Mbps. The end-to-end delay curve for this case is shown in figure 12.

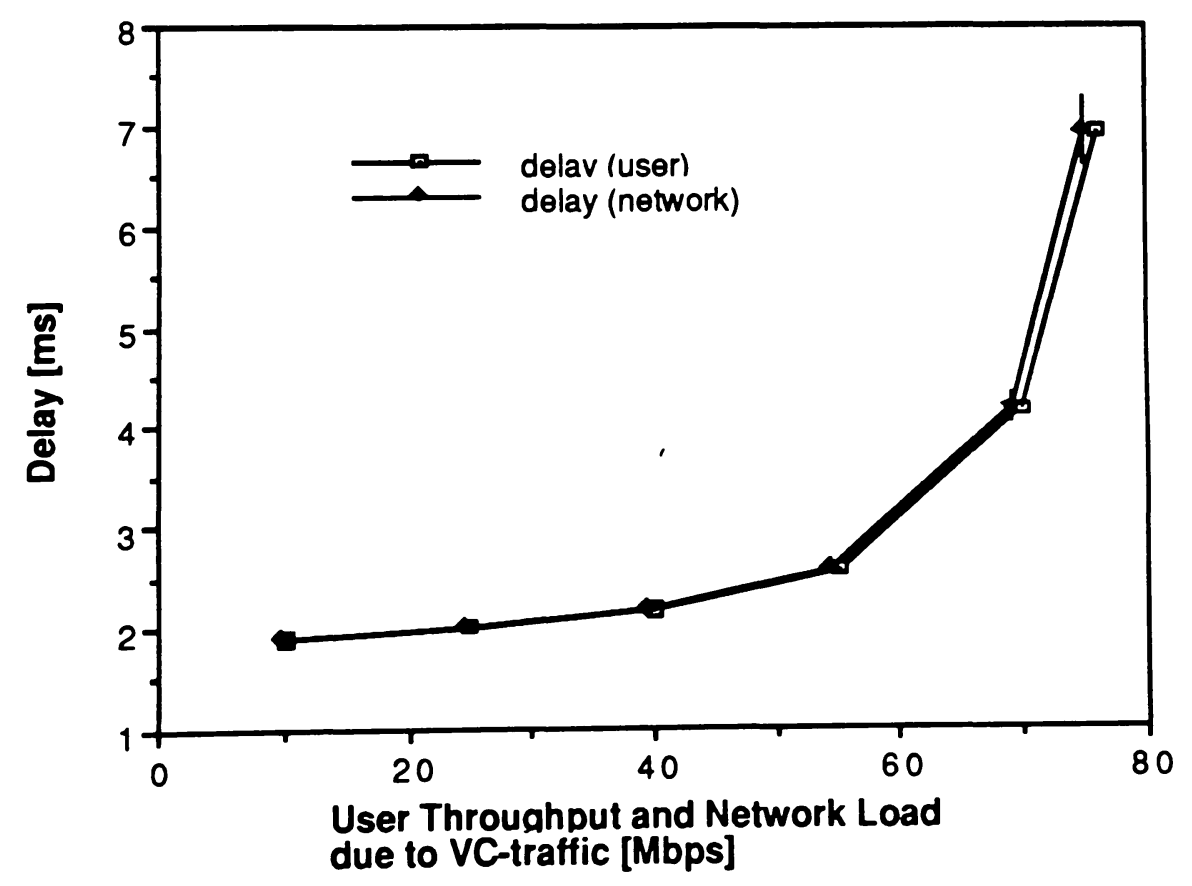

Figure 12. Mean end-to-end delay as a function of user throughput and network load : topology1

13 IPP (Interrupted Poisson Process) is a special case of a two-state MMPP in which the arrival rate in one of the states is equal to zero. 
Figure 13 compares the end-to-end delay for the Poisson, MMPP and IPP arrivals shown in figures 6 and 8 and 12 .

- $\quad$ VC-traffic and External traffic: $\quad$ Poisson, MMPP, IPP

Recall that the external traffic is $20 \mathrm{Mbps}$ in all cases. As the burstiness of the VC-arrivals increases, in this example from 1.915 (MMPP curve) to 10 (IPP curve), the end-to-end delay increases. The increase is significant in the region of high network utilizations.

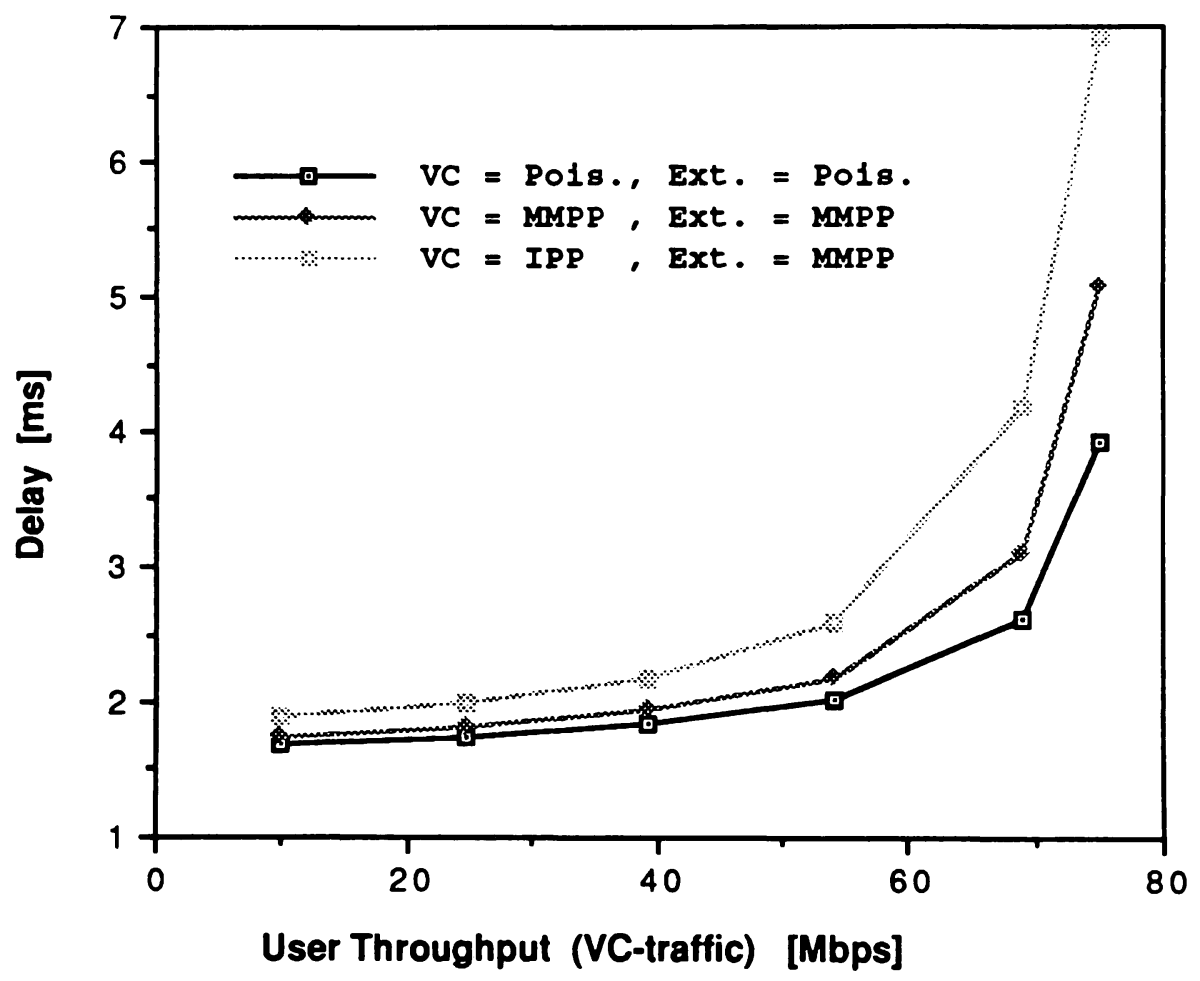

Figure 13. Mean end-to-end delay as a function of user throughput: Poisson, MMPP, IPP arrivals, topology1

Figure 14 shows the mean buffer requirements at networks 1,2 and 3 for the above cases of Poisson, MMPP and IPP arrivals of the VC-traffic. Similarly to the end-to-end delay results, the increase in burstiness leads to higher buffer requirements at the intermediate networks, particularly in the region of high network utilizations. 


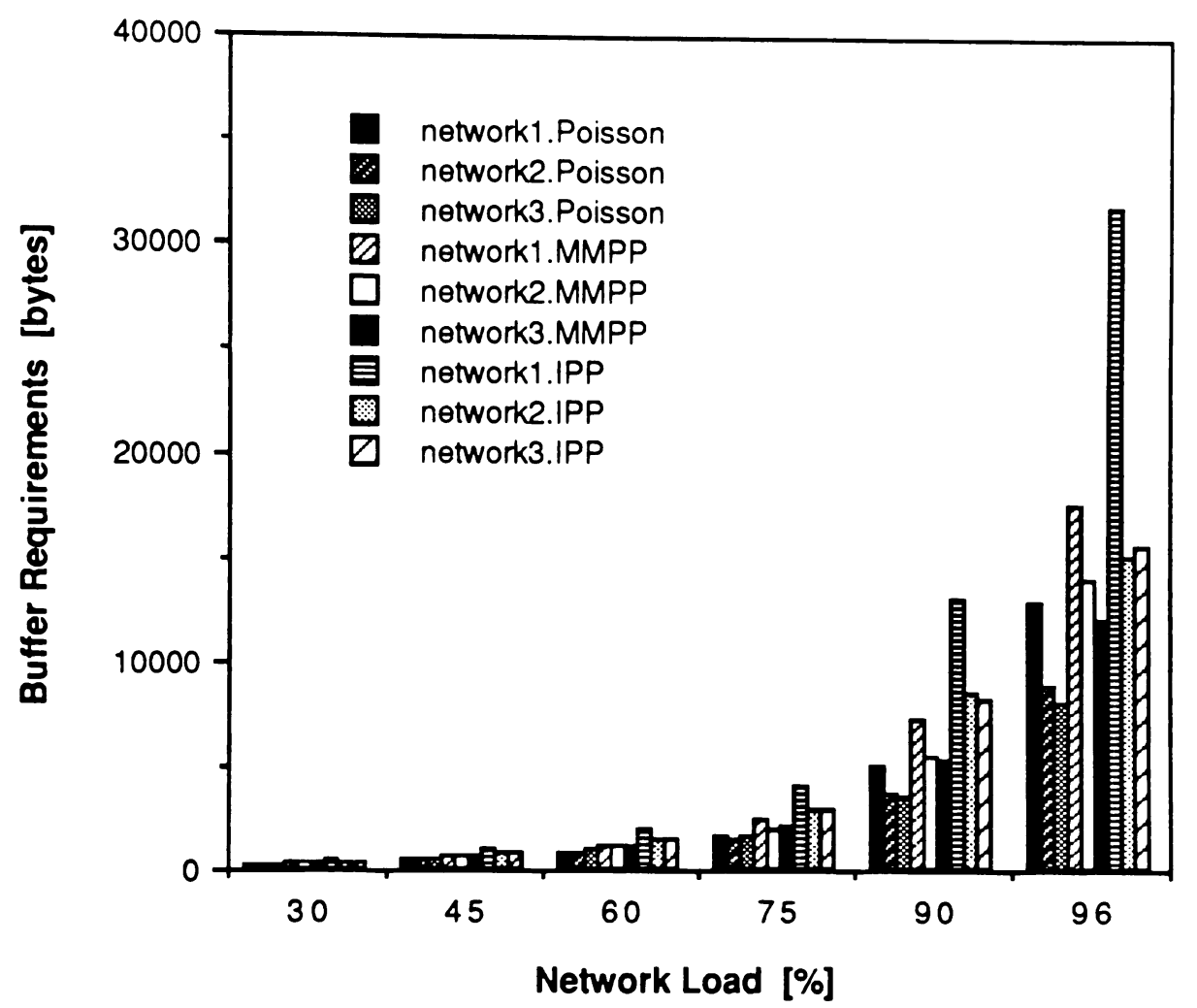

Figure 14. Mean buffer requirements at networks 1,2,3 as a function of total network load : topology 1

In the next two studies we present results for different packet length assumptions:

In figure 15 we demonstrate the effect of the packet length distribution on the end-to-end delay:

- $\quad$ all arrivals $=$ Poisson, $\quad$ External arrivals $=20 \mathrm{Mbps}$

- 'upper' curve:

packet length $=$ 'variable'

- 'lower' curve: packet length $=$ const. 480 bytes 


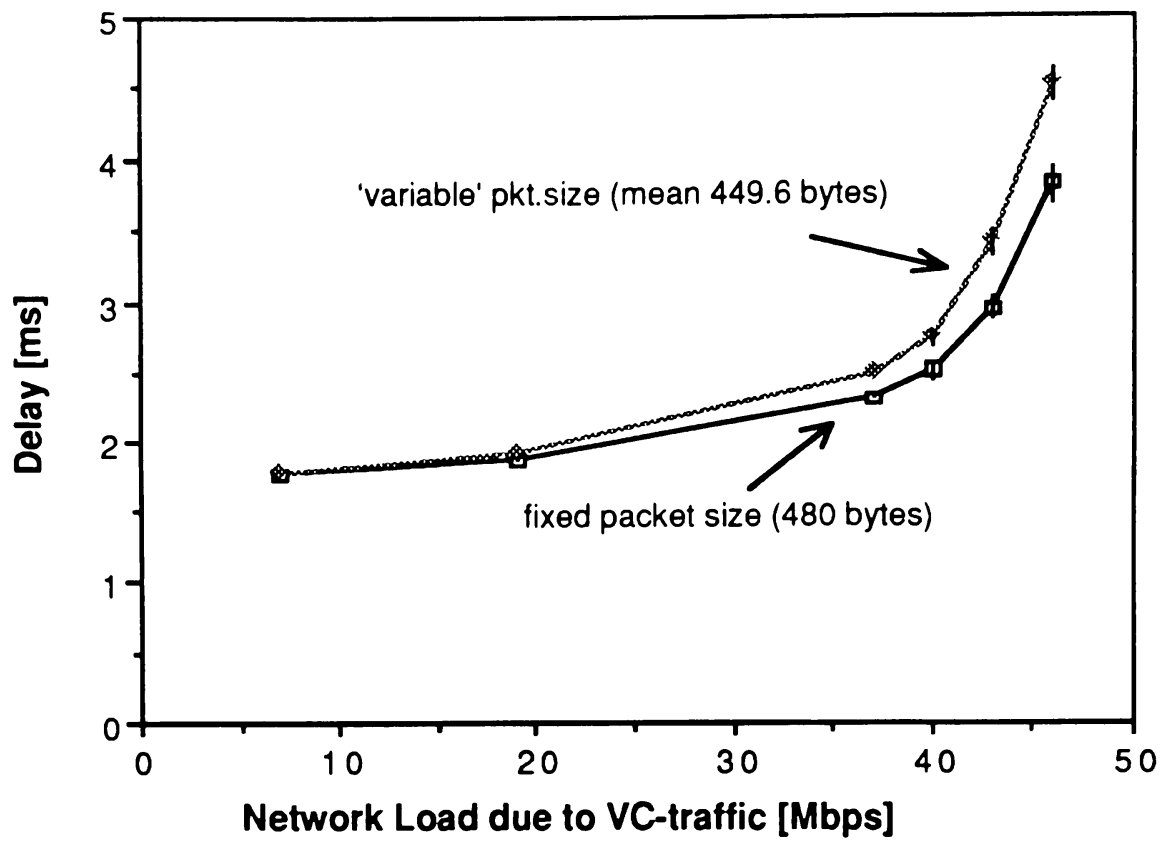

Figure 15. Mean end-to-end delay as a function of user throughput:

Poisson arrivals, const. and variable packet lengths, topology 1

The larger delay in the case of 'variable' packet sizes can be explained using the same arguments as discussed in the previous case, namely, assuming the $M / M / 1$ queue in the case of a 'variable' packet size distribution and a $M / D / 1$ queue in the case of fixed packet sizes.

Figure 16: External arrivals $=20 \mathrm{Mbps}$

- 'upper' curve:

VC-traffic $=$ IPP $\quad$ packet length $=$ const. $(4500$ bytes $)$

Ext.- traffic $=$ MMPP packet length $=$ 'variable'

- 'lower' curve: (same as in figure 12)

VC-traffic $=$ IPP packet length $=$ 'variable'

Ext.- traffic $=$ MMPP packet length $=$ 'variable'

The intention in this study is to evaluate end-to-end packet delay for a bulk file transfer ('upper' curve) under bursty external traffic. It can be assumed that the files are large image files of the size 0.5 Mbytes on average. The image files are split into chunks of 4500 bytes (maximum-size FDDI packets) 


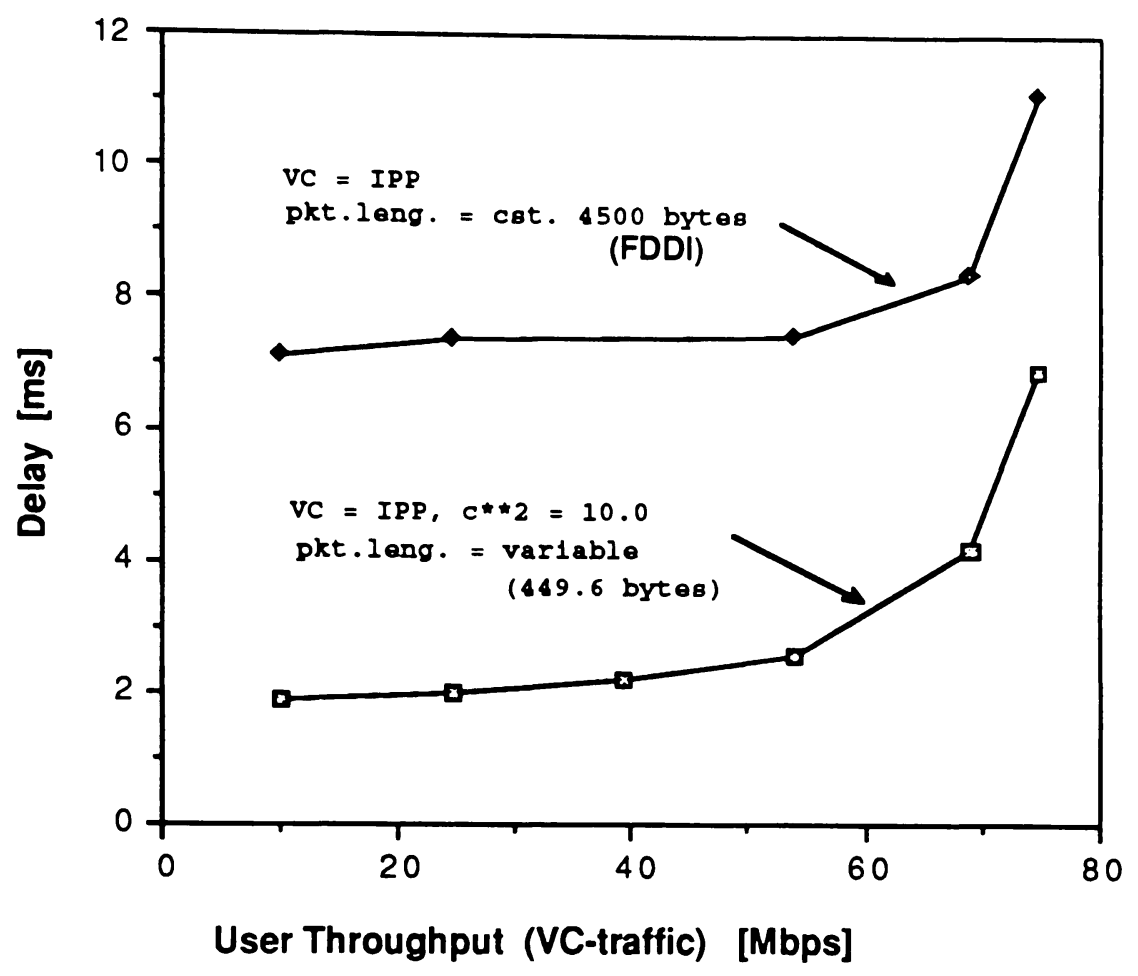

Figure 16. Mean end-to-end delay as a function of user throughput:

IPP arrivals, const. and variable packet lengths, topology 1

prior to reaching the Megacell network. We can assume that an image generating device (user) is attached to a Local Area Network (FDDI network in this case) and the segmentation of images into 4500 byte packets is done by a FDDI network. The packets are transmitted, in-turn, via a Megacell network. We measure here only the delay for individual 4500byte packets crossing the three Megacell networks, not the delay on a per image basis.

The arrival process of a 4500 bytes packet to a Megacell network is assumed to be IPP. The length of an active IPP state is chosen to be $50 \mathrm{~ms}$ (on average), so that one burst represents the arrivals of packets from one image. Namely, during $50 \mathrm{~ms}$, an average number of approx. 1104500 byte packets arrive to the Megacell network, carrying in total 0.5 Mbyte of user information, on average. Recall that in IPP, the duration of each phase ('active' or 'idle') is exponentially distributed. Since time in the 'active' phase is exponentially distributed, and each 'active' phase carries one image, this means basically that the size of an image file is exponentially distributed.

The mean arrival rate in the 'active' state, $\lambda_{1}$, is set to $80 \mathrm{Mbps}, \alpha$ is 0.02 and $\beta$ is calculated for each VC-mean arrival rate. 
The parameters for the IPP-arrivals for the 'lower' curve are described in table 4. The external MMPPs in both curves are the same. The assumptions used in the two curves differ in the parameters of VC-IPPs and packet lengths for the VC-arrivals.

The $c^{2}$ used in the 'upper' curve is shown in figure 17 . The mean VCarrival rate is varied in the range from 10 to $80 \mathrm{Mbps}$, which corresponds to 0.2775 to 2.221 packets/ms ( $x$-axes in figure 17). Hence, the burstiness of the VC-inter-arrival times in the 'upper' curve is much higher than in the 'lower' curve, especially in the region of low VC-arrival rates. The $c^{2}$ in the 'lower' curve is constant, equal to 10 for the entire range of the $\mathrm{VC}$ arrival rates.

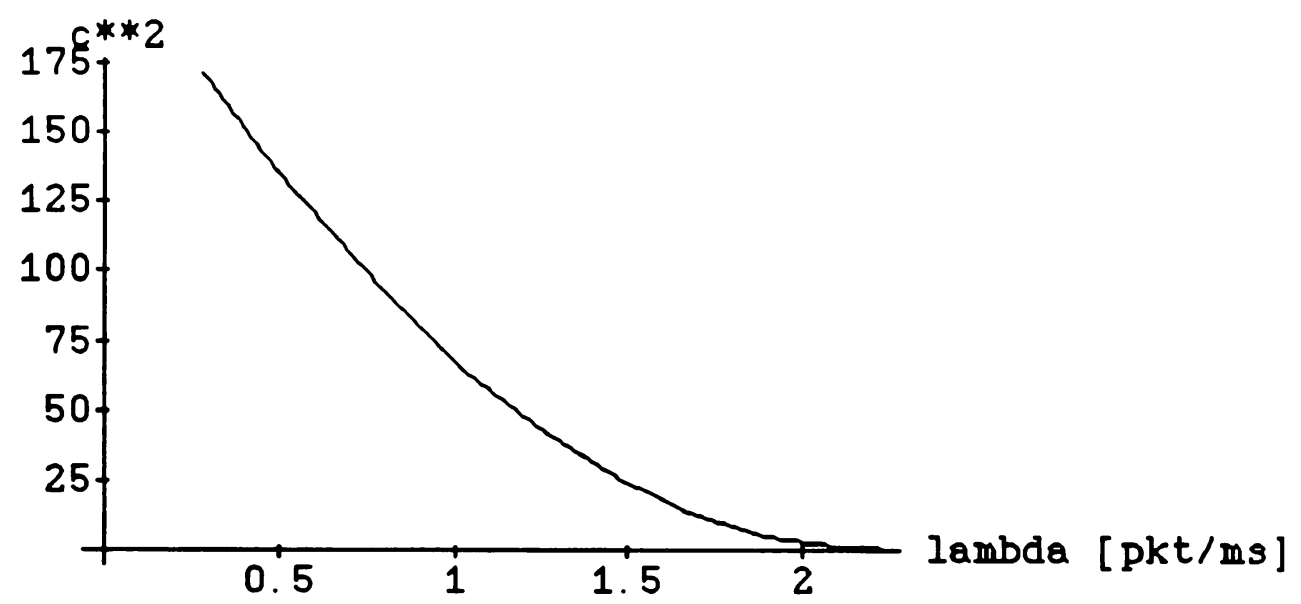

Figure 17. Burstiness $\left(c^{2}\right)$ vs. Mean arrival rate of the IPP

To explain the end-to-end delay results in figure 16 we use an approximate analysis as follows:

Let us first subtract the total propagation delay in both curves $(1.5 \mathrm{~ms})$. The remaining delay consists of a queueing and processing delay. Next, let us approximate the network model with constant 4500-byte packet size for the VC-traffic by a M/D/1 queue ('upper' curve) and the network model with 'variable' packet sizes by a $\mathrm{M} / \mathrm{M} / 1$ queue ('lower' curve) .

If we now take into account that the packet length in the 'upper' network is 10 times bigger, the delay for the 'upper' network should be approximately 5 times bigger (recall, delay in a $M / D / 1$ queue is equal to one half of the delay in a $M / M / 1$ queue). Notice, however, that the delay in the 'upper' curve, after subtracting the propagation delay, is larger by approximately 
10 times in the area of low network utilizations. This may lead us to think that the simulation results in the 'upper' curve are not correct. However, let us go back to figure 16 and recall that for the 'upper' curve we assumed very high burstiness for the VC-arrivals. It becomes apparent now that the large delay in the area of low network utilizations is in fact caused by the high burstiness of the VC-traffic assumed in that region. During the bursts, a large number of packets accumulates in the queues, which leads to larger end-to-end packet delays even under low network utilizations.

This example reflects a possible real situation concerning the bulk filetransfer in the Megacell network. The VC-traffic may be very bursty, as demonstrated in this example. The external traffic is assumed to be less bursty due to the fact that it represents an aggregate traffic from multiple connections. There, we assume that the burstiness essentially 'smoothens out' prior to interacting with the VC-traffic.

We show, again, that the burstiness of network traffic is a very important factor in the end-to-end delay measures. 
$\begin{array}{ll}\text { un } & \text { un } \\ 0 & 0 \\ 0 & 0 \\ 0 & 0 \\ 0 & 0 \\ 0 & 0 \\ 0 & 0\end{array}$

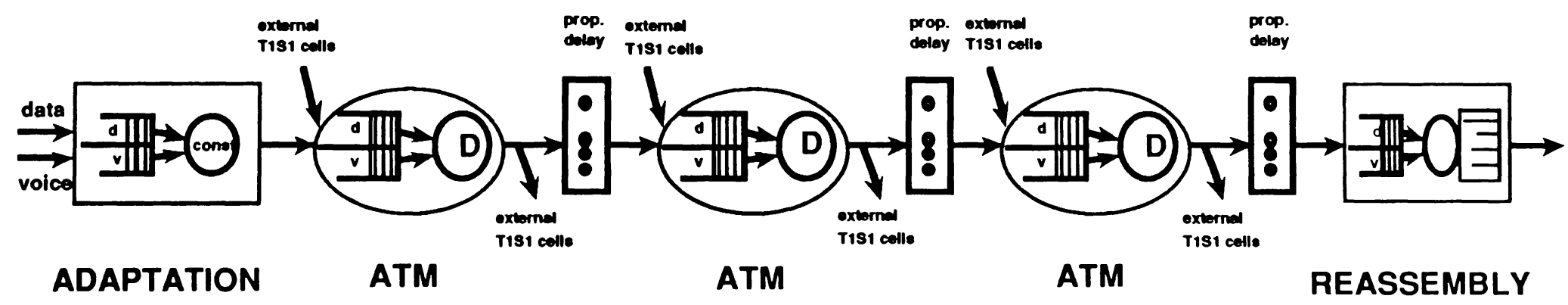




\subsubsection{Discussion of results}

In this section we are going to present only the most representative cases for the topology 2. Other results for this topology will be shown in the next section where we compare the performance of the topologies 1 and 2 .

Since our goal is to compare the two approaches (ATM and Megacell), we made all the assumptions concerning arrival processes, packet length distributions etc. used in the studies in this section the same as for the corresponding cases discussed earlier for topology 1 . We present here only the results for the cases in which we assumed 'variable' 14 packet length distribution for both, the VC-and Ext. traffic.

In the first study external arrivals are $20 \mathrm{Mbps}$ to each network. The assumptions are the same as for the 'MMPP case', discussed in figure 8 topology 1 .

- $\quad$ VC-traffic and External traffic: $\quad$ MMPP

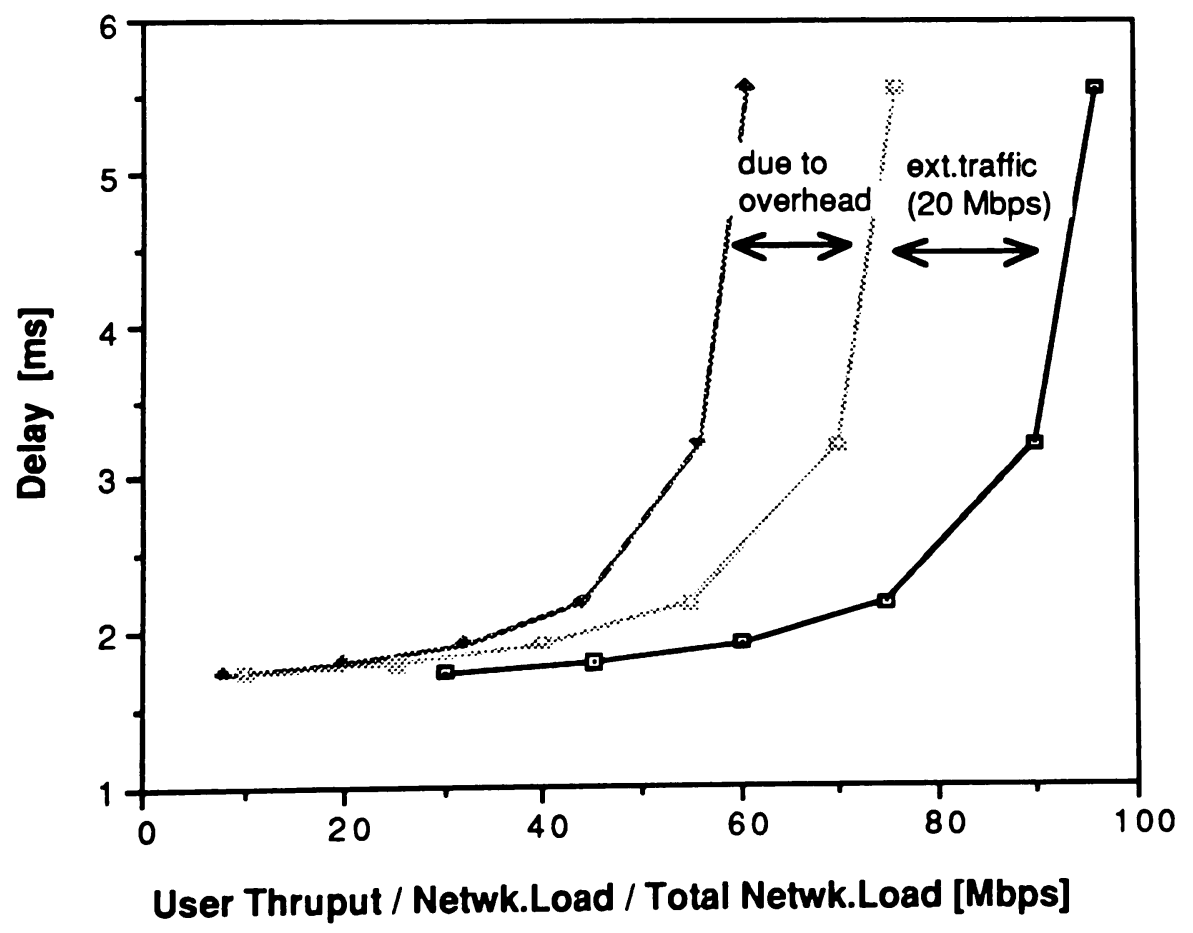

Figure 18. Mean end-to-end delay as a function of user throughput and network load due to VC-traffic and the total network load : topology2

14 Distribution of packet sizes is shown in Section 3.7, figure 5 
Figure 18 shows the end-to-end packet delay as a function of user throughput (left), network load due to the VC-traffic (center) and total network load including $20 \mathrm{Mb} / \mathrm{s}$ external traffic (right).

Recall that the overhead in the ATM network is 9 bytes per ATM cell in the case of no padding bits added for the unfilled cells. Since the average packet length for the 'variable' packet length distribution is 449.6 bytes, and the average overhead is 112.2 bytes/packet, the percentage of overhead in ATM network turns out to be very large $(112.2 / 449.6=24.96 \%)$; consequently, the expansion factor in this case is 1.25 . In the ideal case, where the lengths of user packets are exact multiples of 44 bytes (no padding bits), the overhead is still large ( 9 bytes / 53-bytes ATM cell = $16.98 \%)$. Therefore, the throughput for the user traffic in the ATM network is significantly lower than the offered network load, as demonstrated in figure 18.

In figure 19 we present the comparison of the end-to-end delay results for the cases:

- $\quad$ VC-traffic and External traffic:

Poisson, MMPP

and use the same assumptions that were used in topology 1 for those studies ${ }^{15}$.

Recall that the external traffic is $20 \mathrm{Mbps}$ in all cases. As the burstiness of the VC-arrivals increases, the end-to-end delay increases. The increase is significant in the region of high network utilizations, as demonstrated also in the topology 1.

15 In order to make comparison of the two topologies for the same traffic assumptions one needs to make a choice of what parameters can be kept the same since the average packet lengths due to different overhead in ATM and Megacell network are different. We made a comparison of the two topologies on the basis of the same offered network load due to the VC-traffic (in Mbps). Since the average packet length in the case of an ATM network is larger ( 561.8 bytes compared to 456.1 bytes in Megacell network), the average number of packet arrivals in a unit-time period is smaller in an ATM network for the same offered load in Mbps. Therefore, keeping all the assumptions concerning the $a$ and $b$ parameters of the MMPP or IPP process the same and computing $\lambda_{1}, \lambda_{2}$ of an ATM-MMPP to correspond to the desired arrival rates in Mbps yields to different $c 2$ values in the case of an ATM network. 


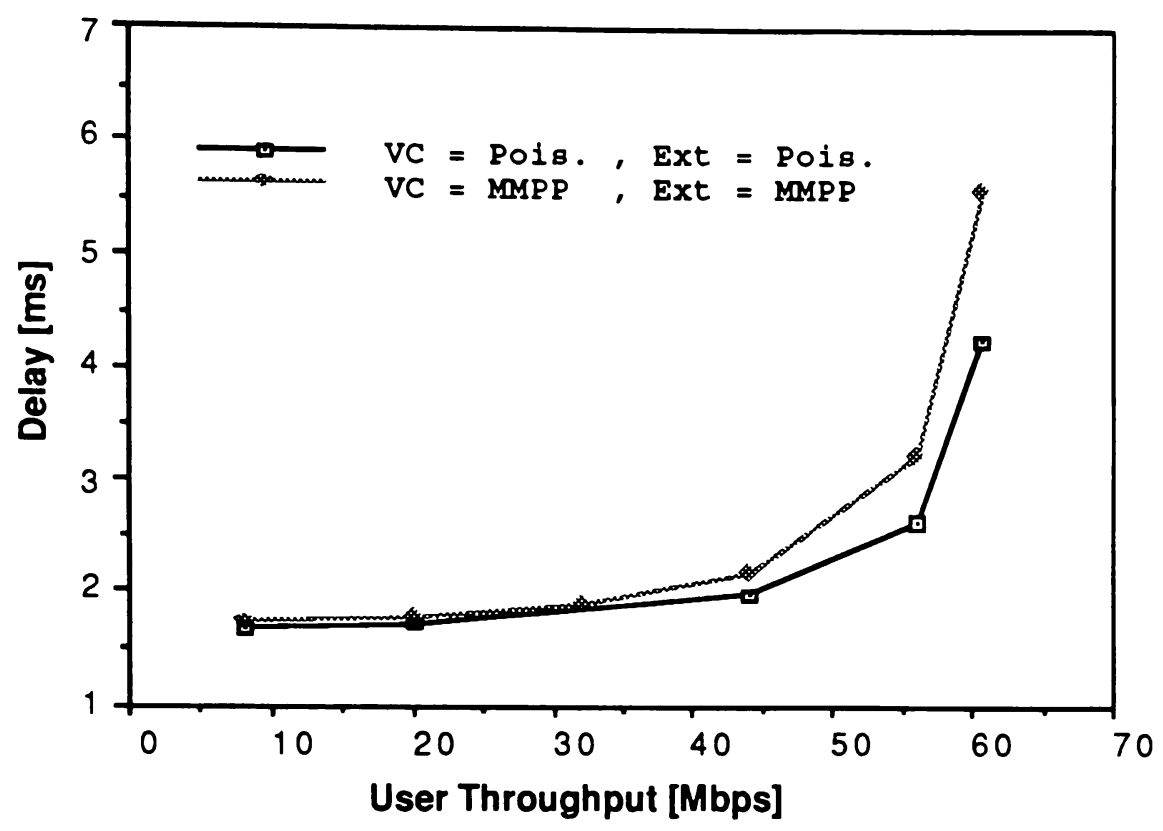

Figure 19. Mean end-to-end delay as a function of user throughput: Poisson and MMPP arrivals, topology2

In figure 20 we show the end-to-end delay for the case:

- VC-traffic: Poisson External traffic: Poisson 50 Mbps

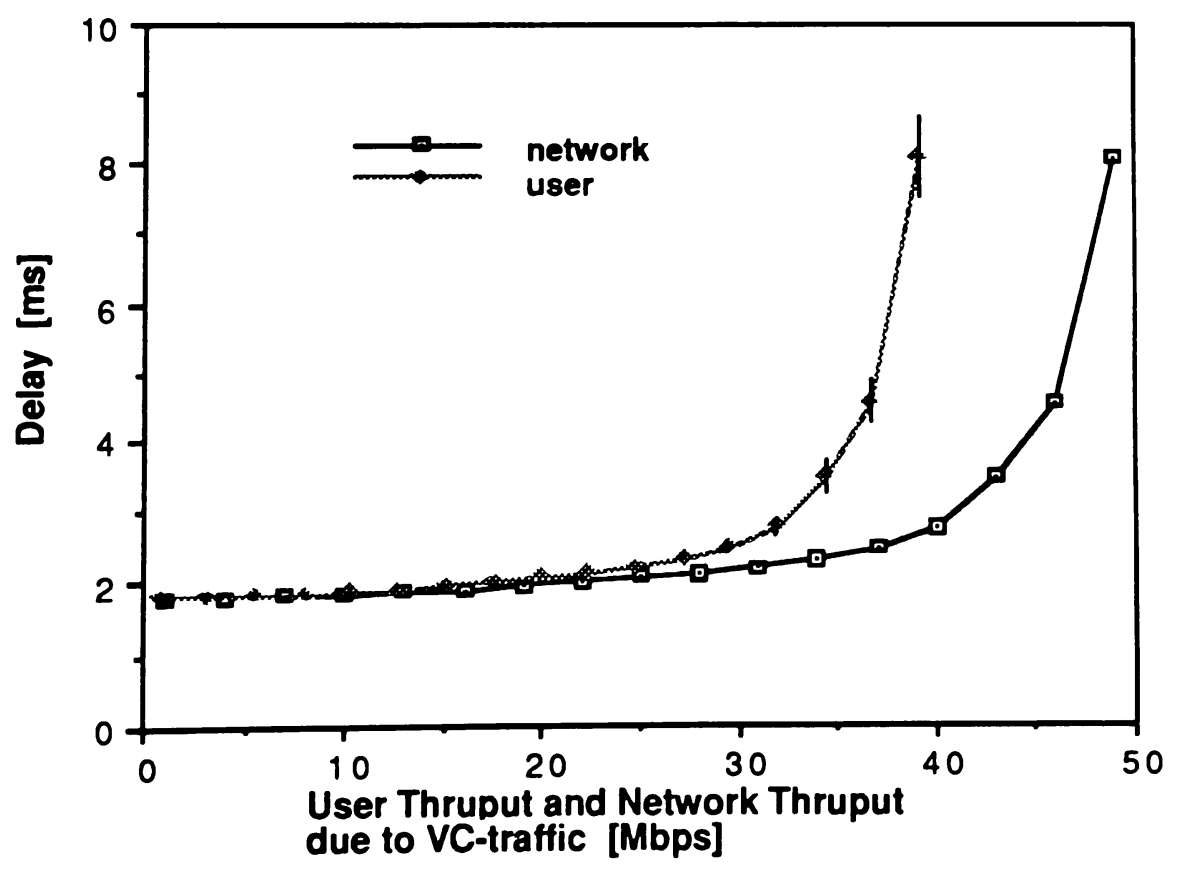

Figure 20. Mean end-to-end delay as a function of user throughput and network load : topology2 
Finally, we present two cases with different assumptions than those used in the majority of the studies.

In figure 21 we present the end-to-end delay for:

- VC- and External traffic :

Poisson (same as in figure 20)

Buffers: fin it e

30 kbytes, 40 kbytes

Recall that packet length is 'variable', external traffic is $50 \mathrm{Mbps}$ in figures 20 and 21. Due to finite buffers in network 2 and 3 cells occasionally get lost. Each time a cell loss occurs, the entire packet is retransmitted. The retransmission request is issued in the 'Reassembly' module (destination's Adaptation layer). The details concerning the packet retransmission are given in section 2.5 .

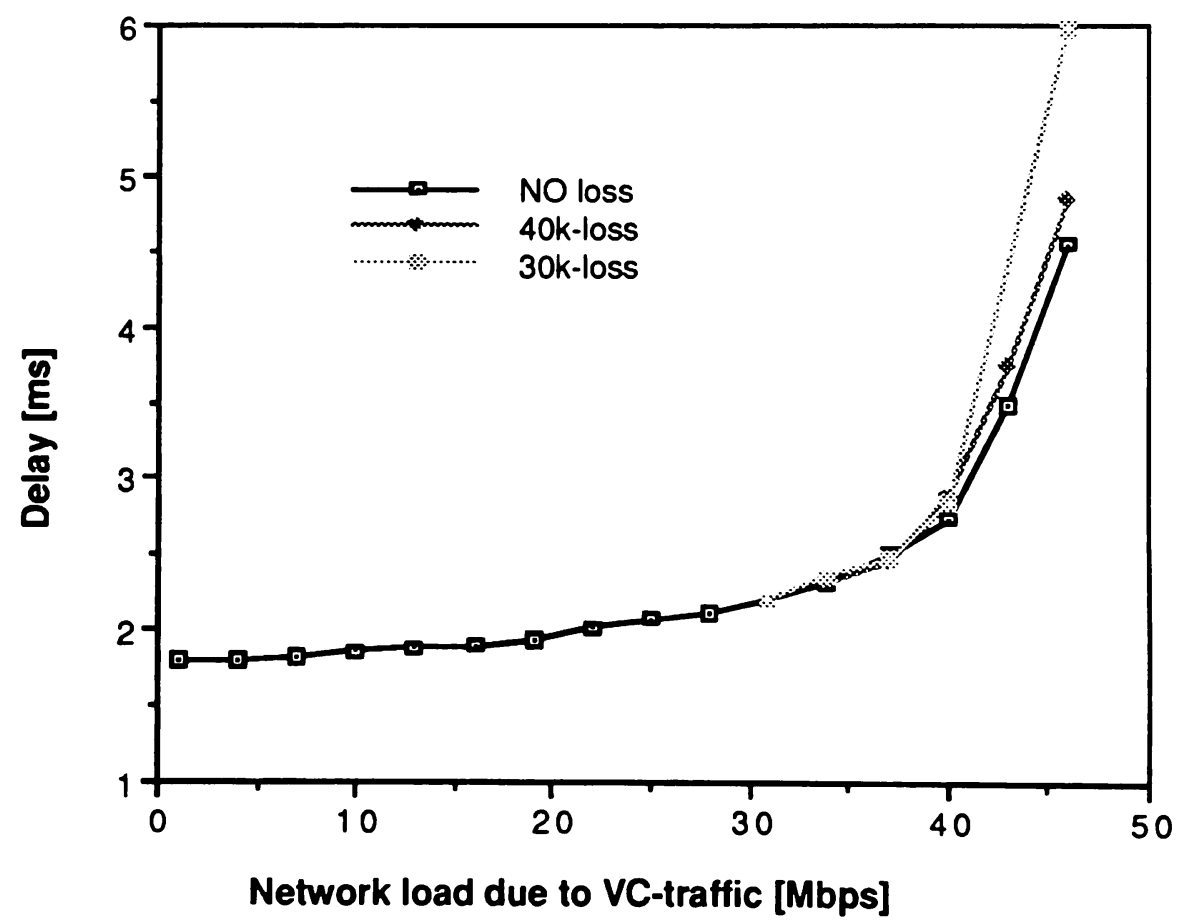

Figure 21. Mean end-to-end delay as a function of network load, finite buffers at networks 2 and 3, topology2

The cell loss occured only under high network utilizations and caused an increase in average packet delay. The percentage of lost packets in the case of 40 kbyte buffers at networks 2 and 3 was $1.9 \%$. In the case of a real network implementation, this value for the packet loss is too high. However, the intention in this study was to demonstrate the effect of finite buffers on the average end-to-end packet delay. In order to obtain 
noticeable increase in average packet delay and keep the simulation runs reasonably long, we had to chose lower values for the buffer sizes in networks 2 and 3.

To determine the suitable buffer sizes we have first done a simulation run with infinite buffers and evaluated buffer requirements for the case of no cell loss (i.e. the maximum buffer requirements). Then, we determined the finite buffer sizes for the following experiments. The size for the buffers had to be chosen carefully, since if the buffer sizes were chosen too small, the network would easily be saturated. Due to the fact that a large number of cells would be lost, the number of retransmission packets would become large which would, in-turn, increase the cell loss probability, and so forth. (The load due to the retransmissions is an additional load to the normal load of the VC- and ext.traffic.)

Figure 22 gives the mean buffer requirements for the case of no cell loss, shown in figure 21 . In general, maximum buffer requirements depend on burstiness of the network traffic. In this particular case, the arrivals are Poisson and the maximum buffer requirements are not significantly greater than the mean requirements.

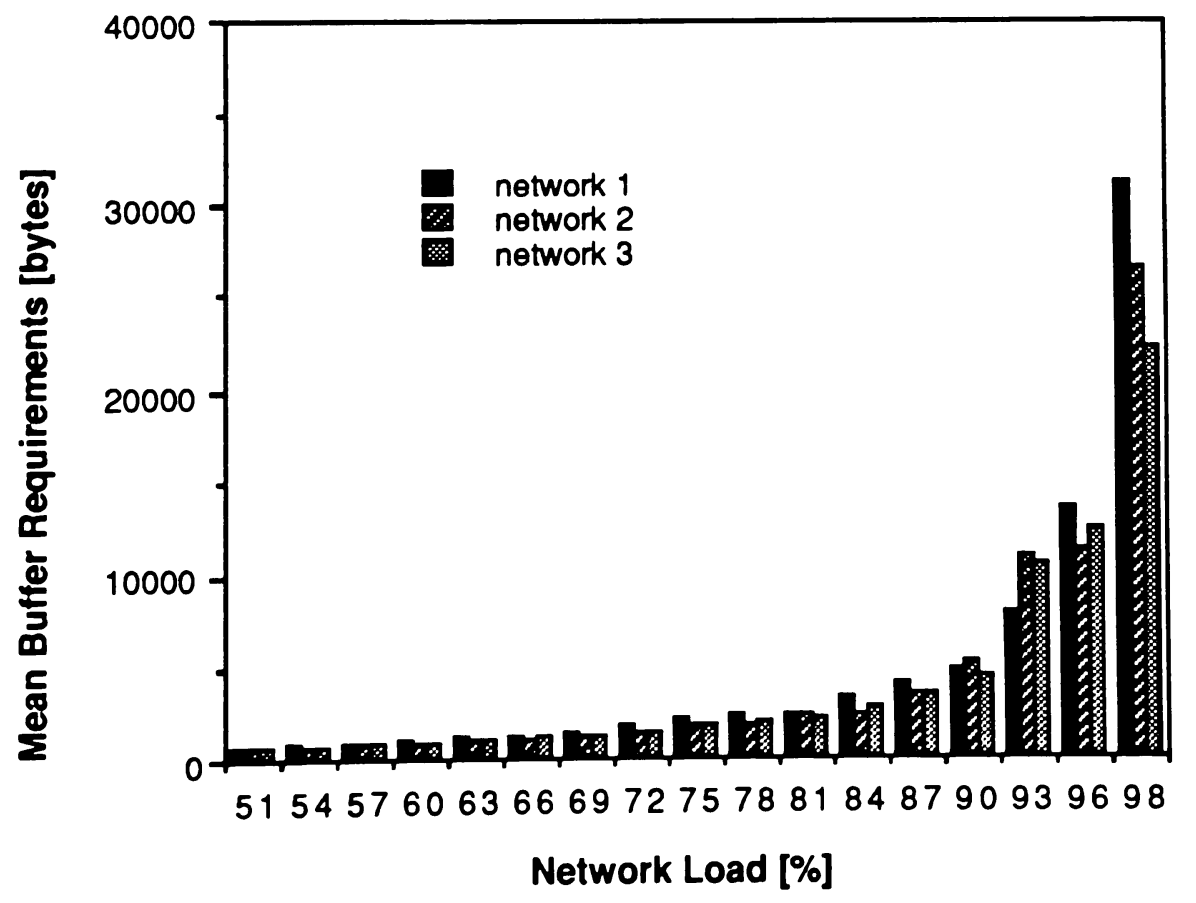

Figure 22. Mean buffer requirements for networks 1,2 and 3 in the case of no cell loss, external traffic $=50 \mathrm{Mbps}$, topology 2

In the last study we present the results for two classes of traffic. The 'high priority' traffic (denoted as 'voice' in the block diagrams) has higher 
priority at each server. 'Low priority' traffic is served only if there is no 'high priority' packets in a queue. The details on the implementation of the priorities in the models are given in section 2.1.

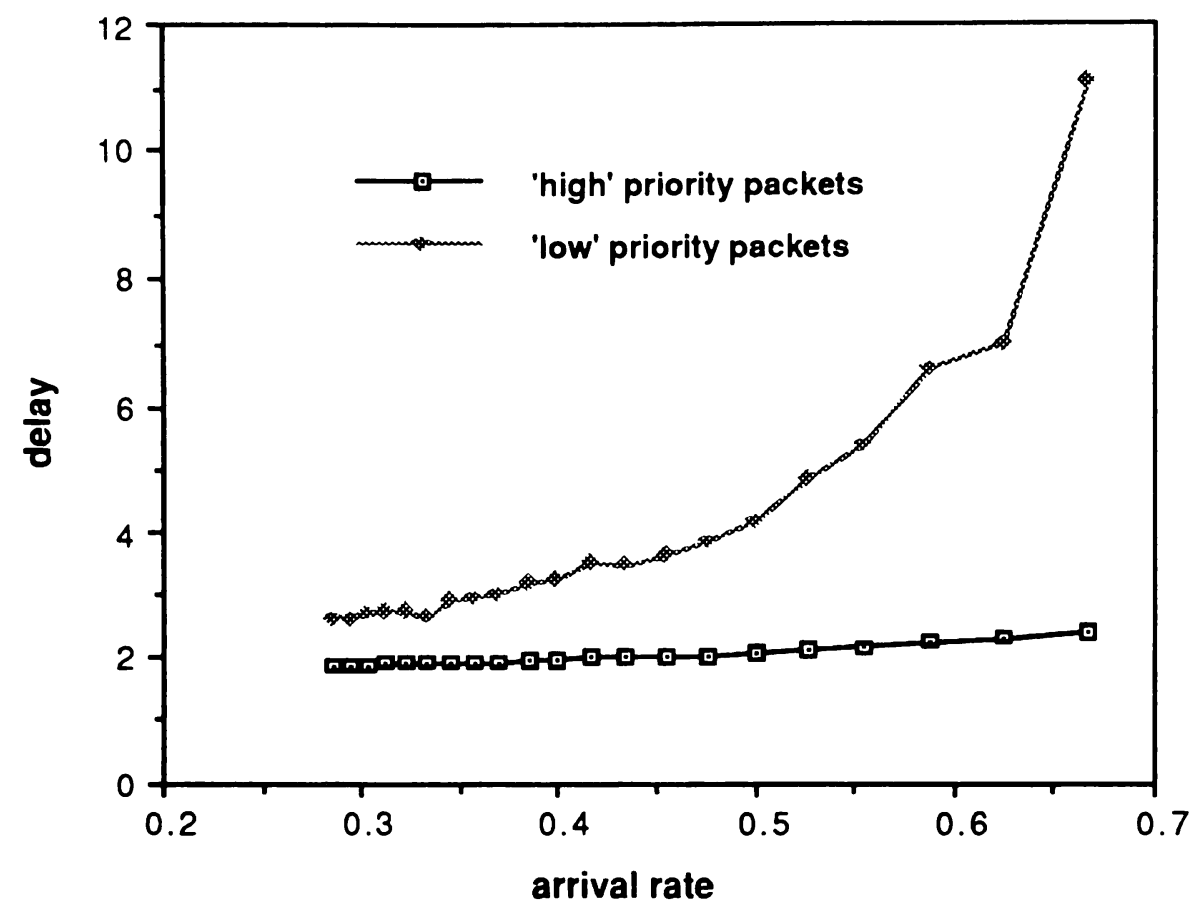

Figure 23. Mean end-to-end delay for 'high' and 'low' priority packets (in simulation time-units)

The model parameters used in this particular study are arbitrarily chosen ${ }^{16}$ and are not consistent with the assumptions presented in the outline at the beginning of section 5.3. However, the curves give a general idea about the end-to-end delay performance per priority class.

Finite buffers and priority queueing are not treated in-depth in this report, although the simulation programs were written to support both assumptions. The reasons for concentrating in the studies on a single priority class and infinite buffers are explained in section 5.2.

16 adaptation time $=0.5, \mathrm{ATM}$ cell-service time $=0.1$, propagation delay $=0.08$, reassembly cell processing time $=0.02$, all external arrivals $=7.5$, no cell loss 


\subsection{TOPOLOGY 1 AND 2 : A COMPARISON}

In this section we compare the results obtained for topologies 1 and 2 .

In the following figures, packet length distribution is 'variable'17 for both. VC- and External traffic. External traffic is $20 \mathrm{Mbps}$. Arrival processes of the VC-and External traffic are varied:

- $\quad$ VC-traffic and External traffic: $\quad$ Poisson

Figure 24 shows the end-to-end packet delay vs. network load due to the VC-traffic (VC-network load $=$ user data load + overhead ${ }^{18}$ ).

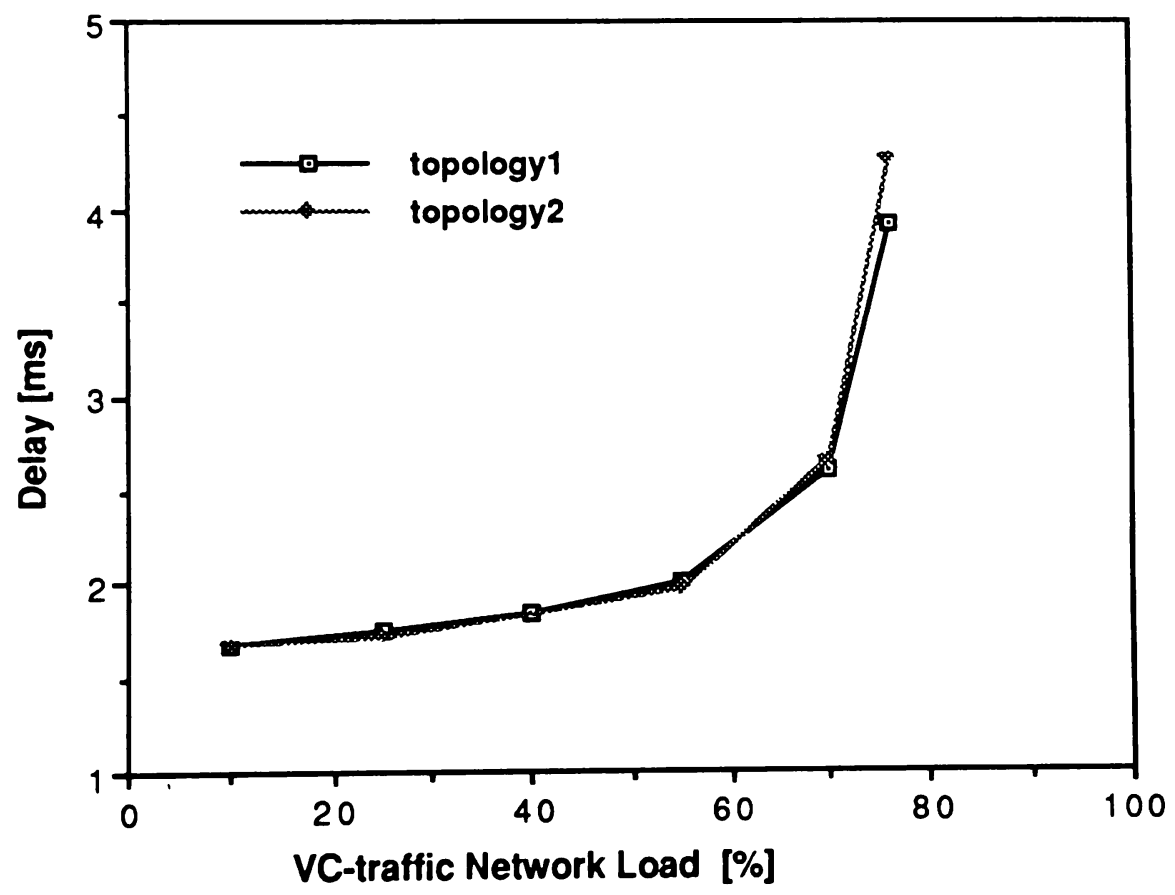

Figure 24. Mean end-to-end delay as a function of network load due to VC-traffic: Poisson arrivals, topology 1 vs. 2

The results from the above curve indicate that both networks transmit a given amount of data (the VC-traffic network load) with approximately the same delay. The delay for the topology 1 is lower under high network utilizations.

17 Distribution of packet sizes is shown in section 3.7, figure 5

18 The amount of overhead in a ATM and Megacell network for the case of a 'variable' packet length distribution is shown in section 5.3 , table 1 
However, rather than comparing the two topologies on the basis of the endto-end delay for the VC-offered load, which includes the load due to the network overhead, the user is more interested in a comparison on the basis of the end-to-end delay for the user packets. Since the overhead in the ATM network is very large ${ }^{19}$, and if the two networks are compared for the same amount of user information transmitted (same 'user throughput'), then the ATM network will have higher delay. As shown in figure 25, the difference in the delay is particularly large under higher network utilizations.

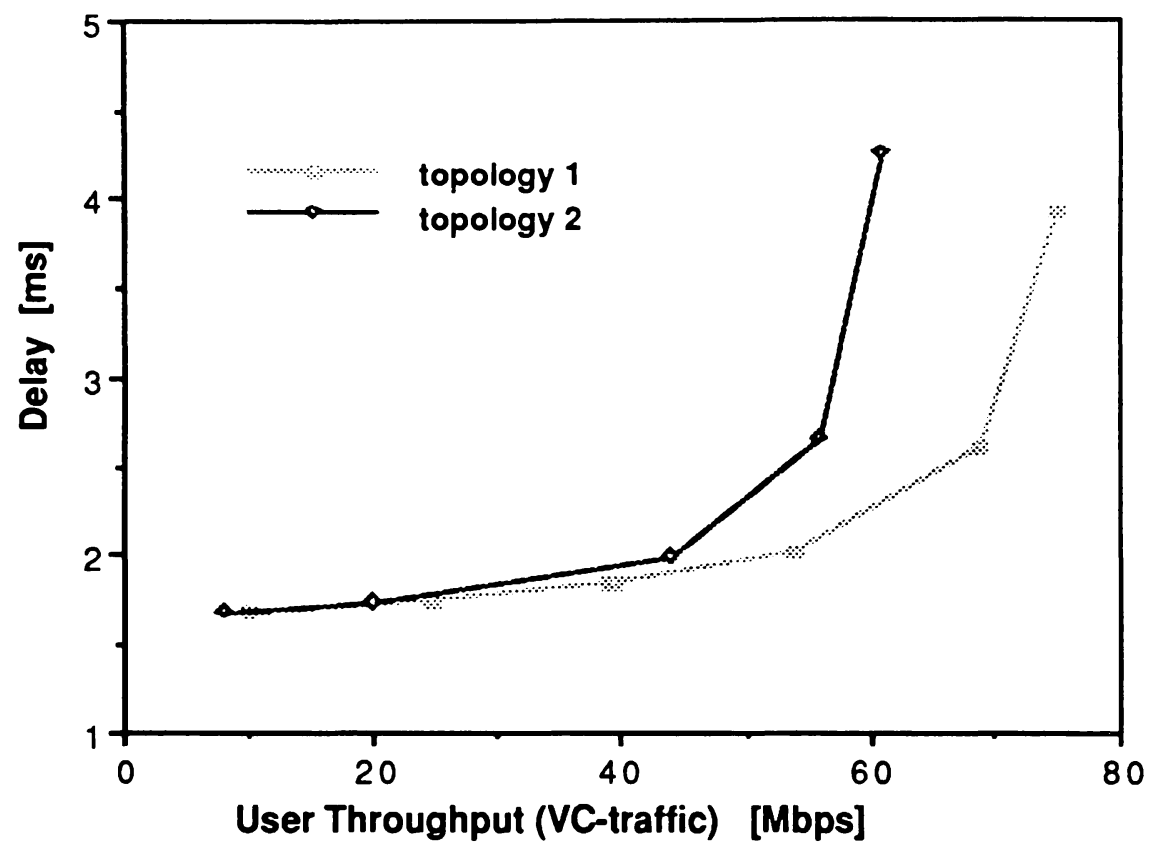

Figure 25. Mean end-to-end delay as a function of user throughput: Poisson arrivals: topology 1 and topology 2

Figure 26 shows the end-to-end delay vs. both, the user throughput and the network load due to VC- traffic. Since the network capacity is $100 \mathrm{Mbps}$ and the external load is $20 \mathrm{Mbps}$, the total available capacity for the VCtraffic is $80 \mathrm{Mbps}$. The overhead in the ATM network for the 'variable' packet length distribution is $25 \%$ of the total amount of data transmitted. Therefore, the maximum user throughput for the ATM network in this case is $60 \mathrm{Mbps}$, compared to almost $80 \mathrm{Mbps}$ in the Megacell network (due to only $1.4 \%$ overhead). As the network speeds increase, the absolute amount of loss in the transmission capacity in the ATM network increases.

19 As discussed earlier, the overhead is at least $16.9 \%$ in the case of no padding bits added for the unfilled cells (see section 5.3 for more details) 


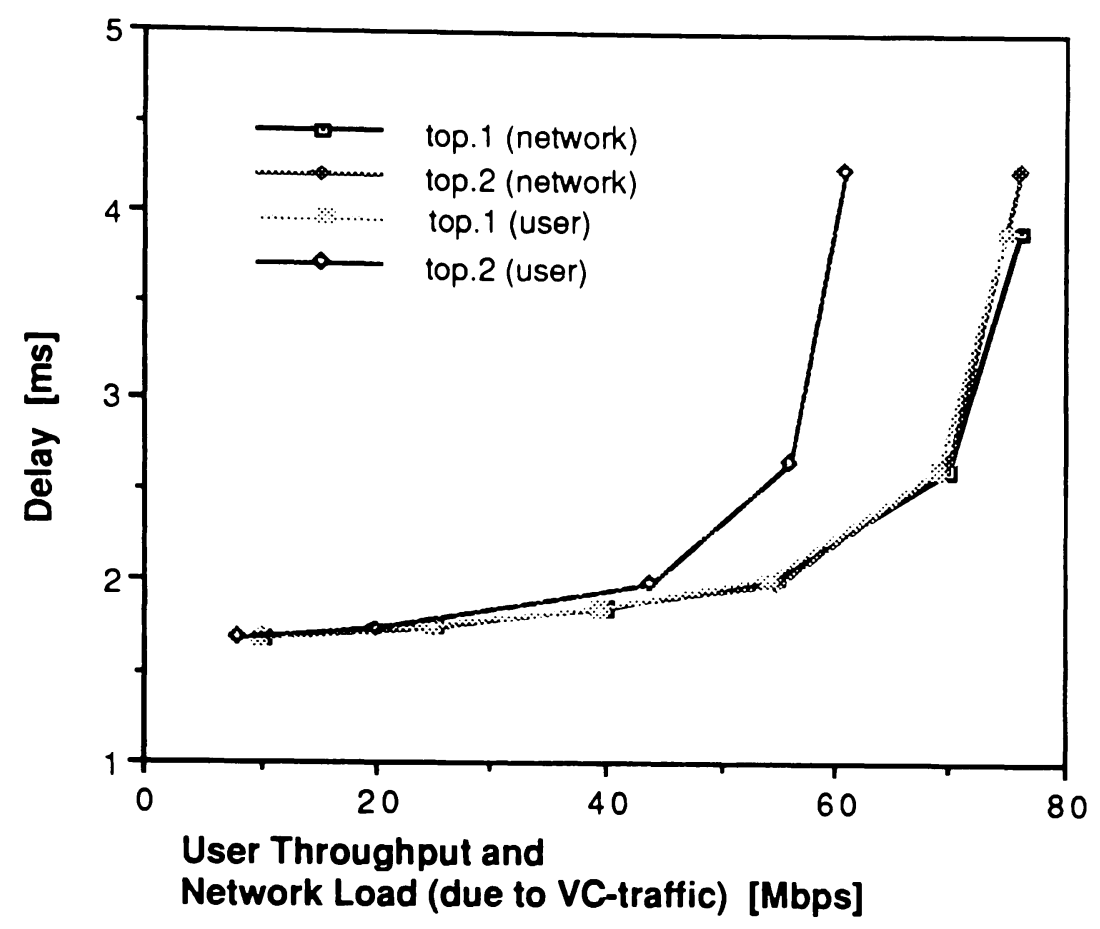

Figure 26. Mean end-to-end delay as a function of user throughput and network load due to the VC-traffic: Poisson arrivals, topology 1 vs. 2

Figures 27-29 represent the cases where:

- $\quad$ VC-traffic and External traffic: $\quad$ MMPP

The parameters used for the VC- and External MMPPs are shown in section 5.3.2, table 3. The end-to-end delay results for the topology 1 and 2 have been discussed in sections 5.3.2 and 5.4.2, respectively. The comparison diagrams with MMPP arrivals are presented here in the same order as for the case of the Poisson arrivals. The end-to-end delay results are similar to those shown for the Poisson arrivals, except that the delays are generally higher due to the burstiness introduced by the MMPParrivals. Higher delays in the case of bursty traffic have already been demonstrated in figures 10 and 19.

Figure 27 shows that the end-to-end delay with respect to the total VCtraffic is approximately the same in both topologies. The burstiness does not favorize any of the two topology in terms of the mean end-to-end delay. 


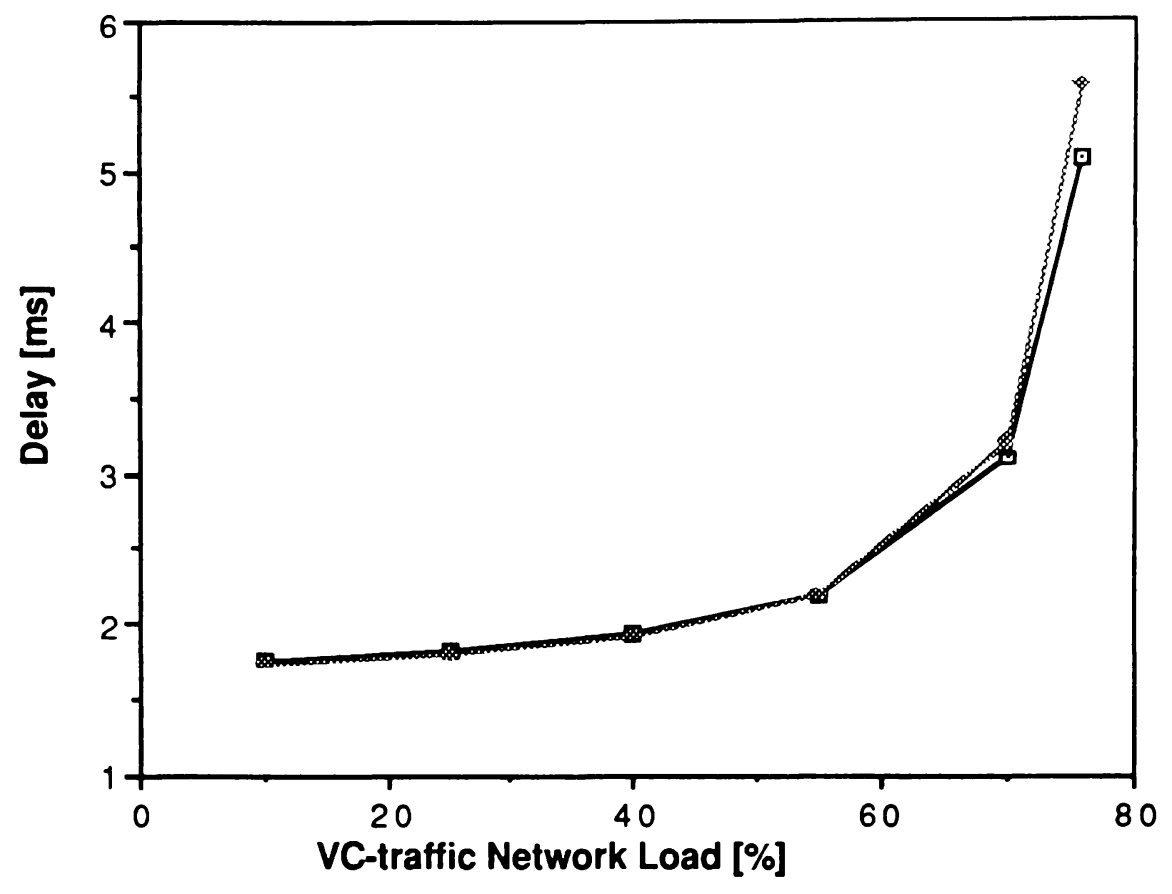

Figure 27. Mean end-to-end delay as a function of network load due to VC-traffic: MMPP arrivals, topology 1 vs. 2

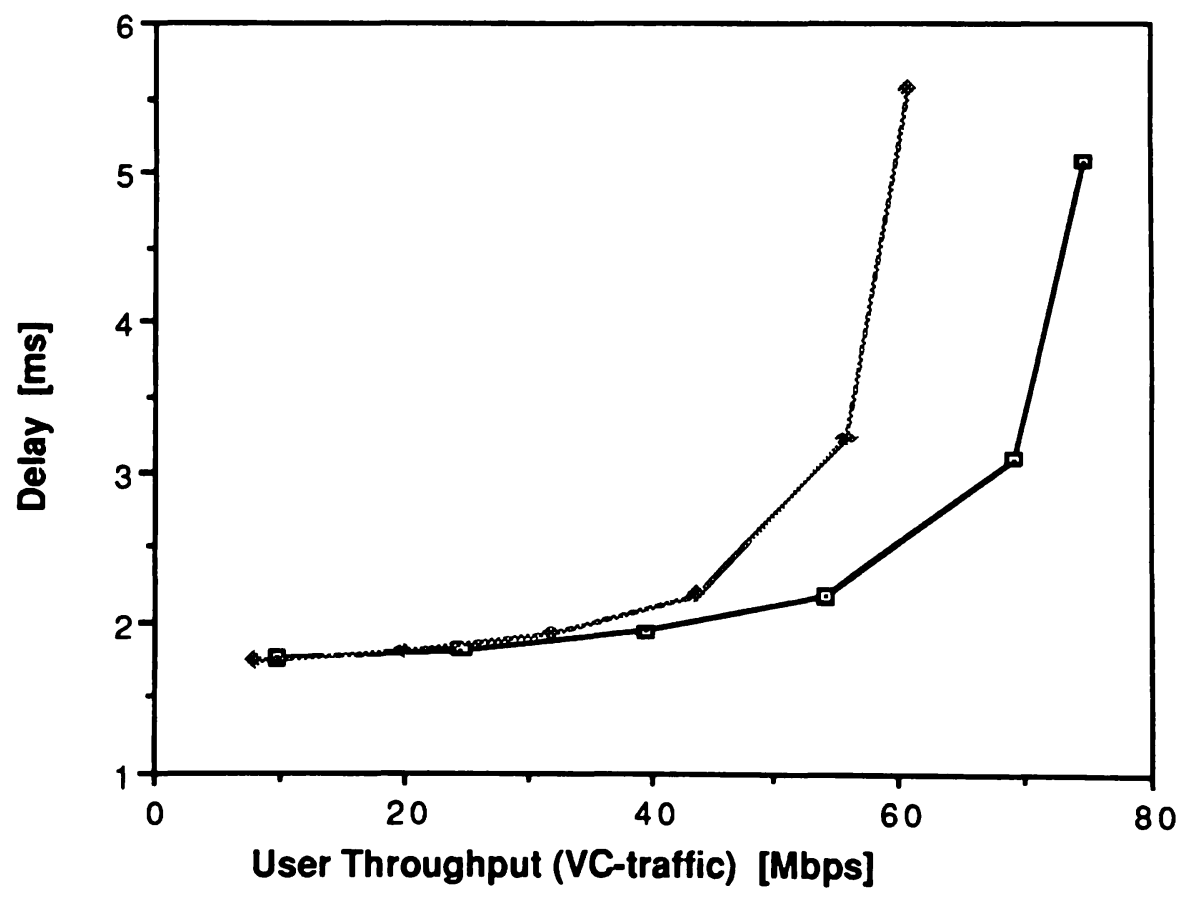

Figure 28. Mean end-to-end delay as a function of user throughput: MMPP arrivals, topology 1 vs. 2 


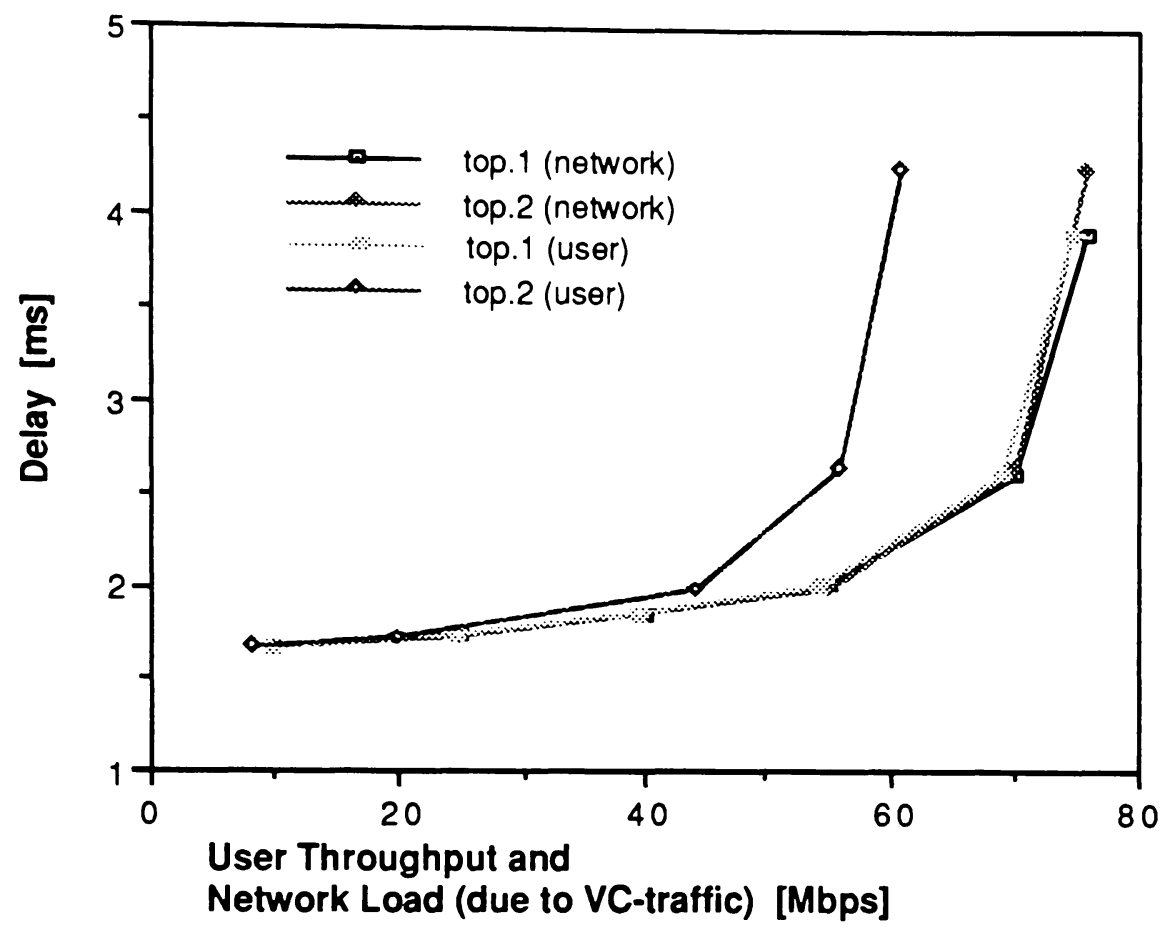

Figure 29. Mean end-to-end delay as a function of user throughput and network load due to the VC-traffic: MMPP arrivals, topology $1 \mathrm{vs.} 2$

Figure 28 shows that the end-to-end delay for the user packets is higher in the case of the ATM network, compared to the case of the Poisson arrivals discussed previously. Figure 29 includes the information from figures 27 and 28.

In the next four figures we present the comparison for the mean and maximum buffer requirements at networks 1,2 and 3 for the case of Poisson and MMPP arrivals. Recall that no cell loss is assumed in these studies.

Figure 30 and 31 show the mean and maximum buffer requirements for the Poisson case and figures 32 and 33 for the MMPP case.

In general, the diagrams do not show any significant advantage in terms of lower mean and maximum buffer requirements for either topology ${ }^{20}$. Notice that mean buffer requirements are the highest in the first network and the lowest in the last network on the end-to-end path.

20 The maximum buffer requirements were obtained for simulation runs of 500,000 packets 


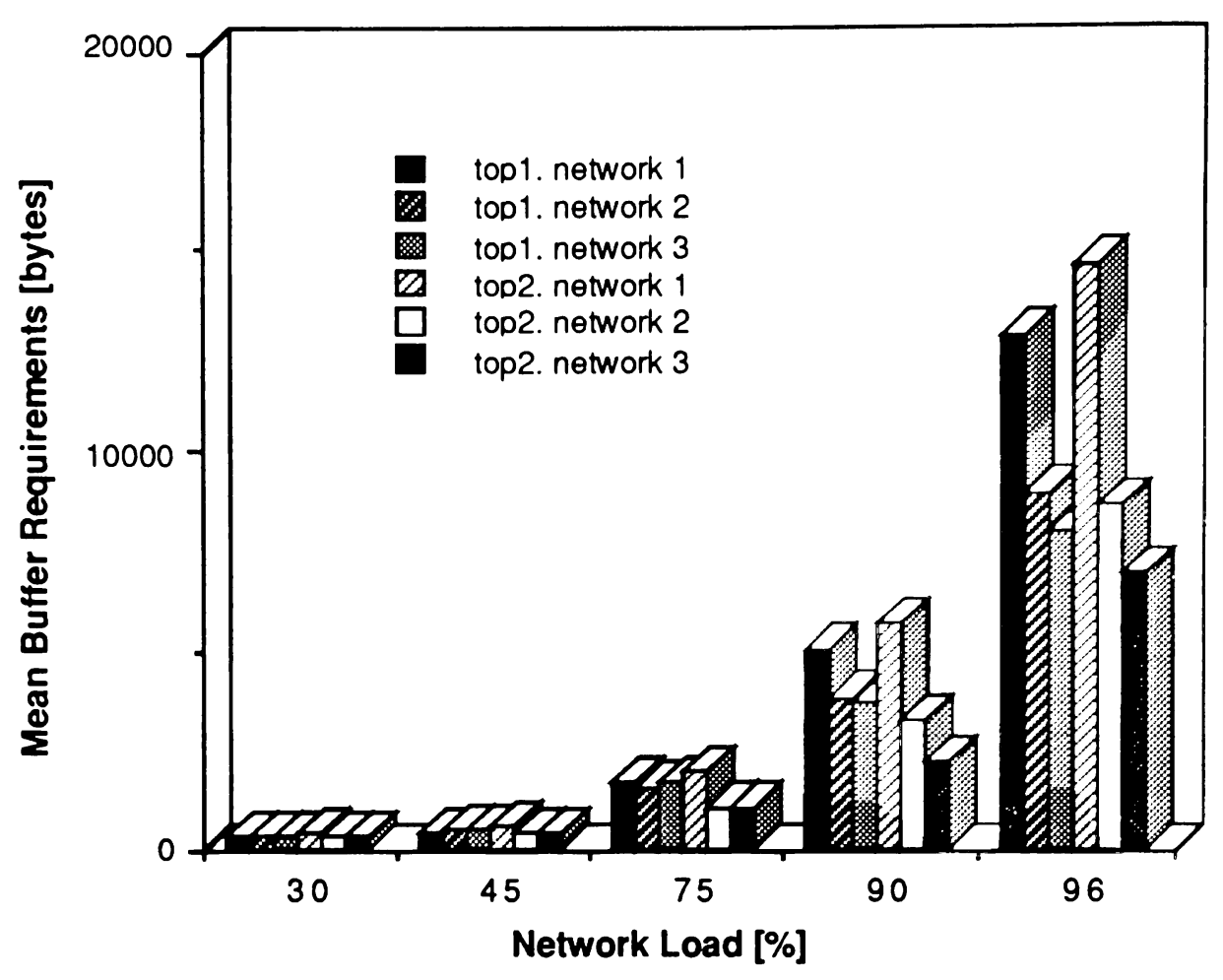

Figure 30. Mean buffer requirements: topology 1 vs. topology 2

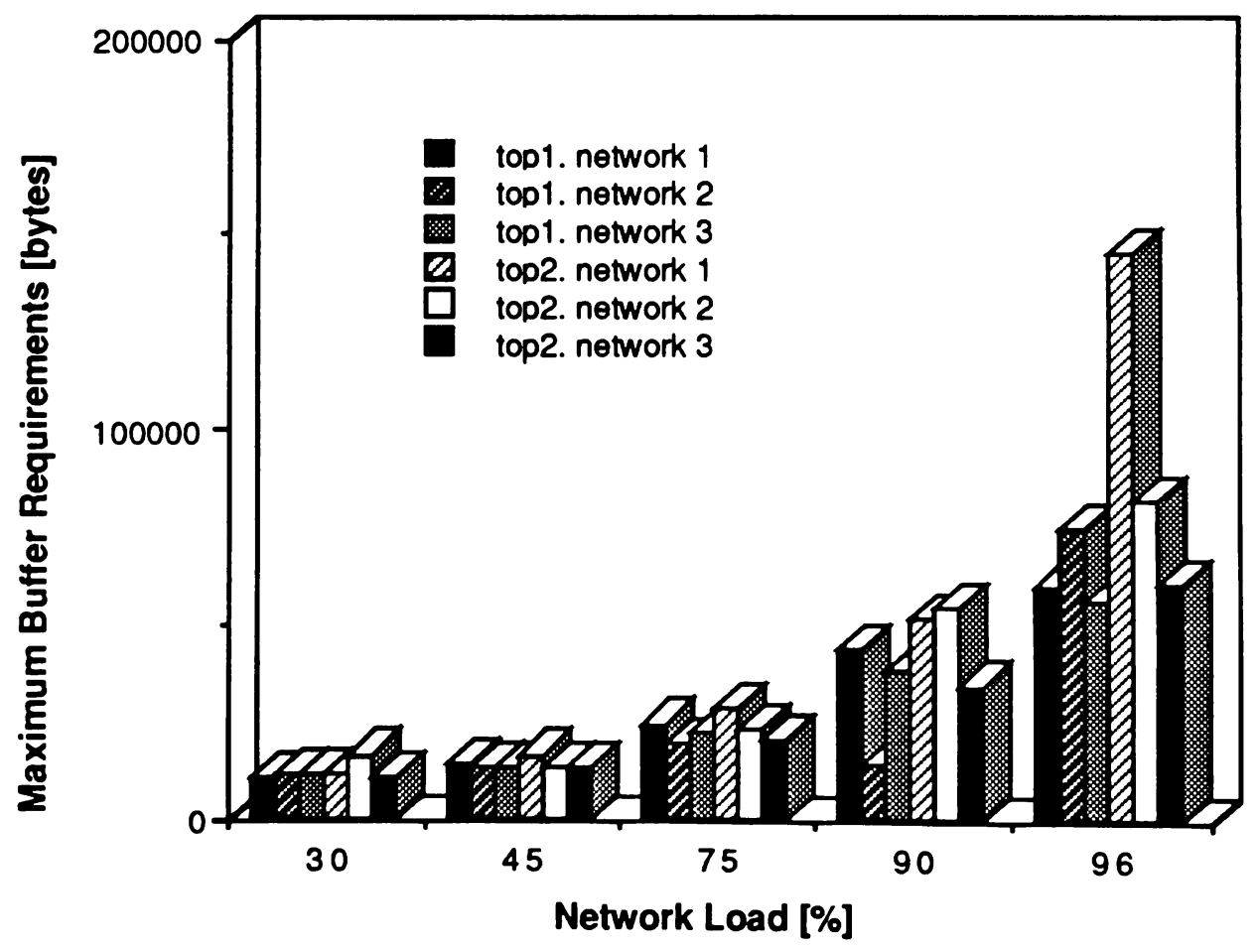

Figure 31. Maximum buffer requirements: topology 1 vs. topology 2 


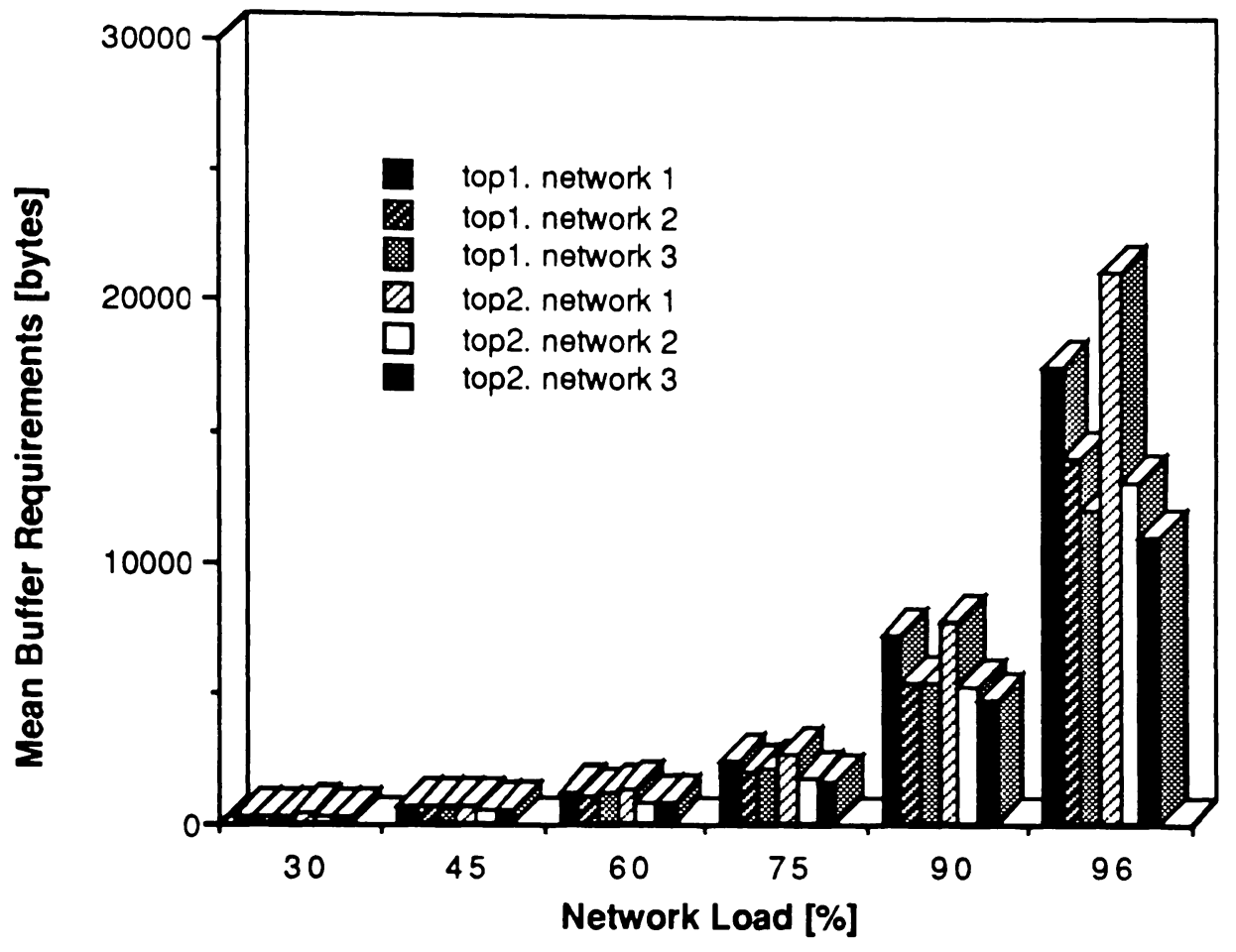

Figure 32. Mean buffer requirements: topology 1 vs. topology 2

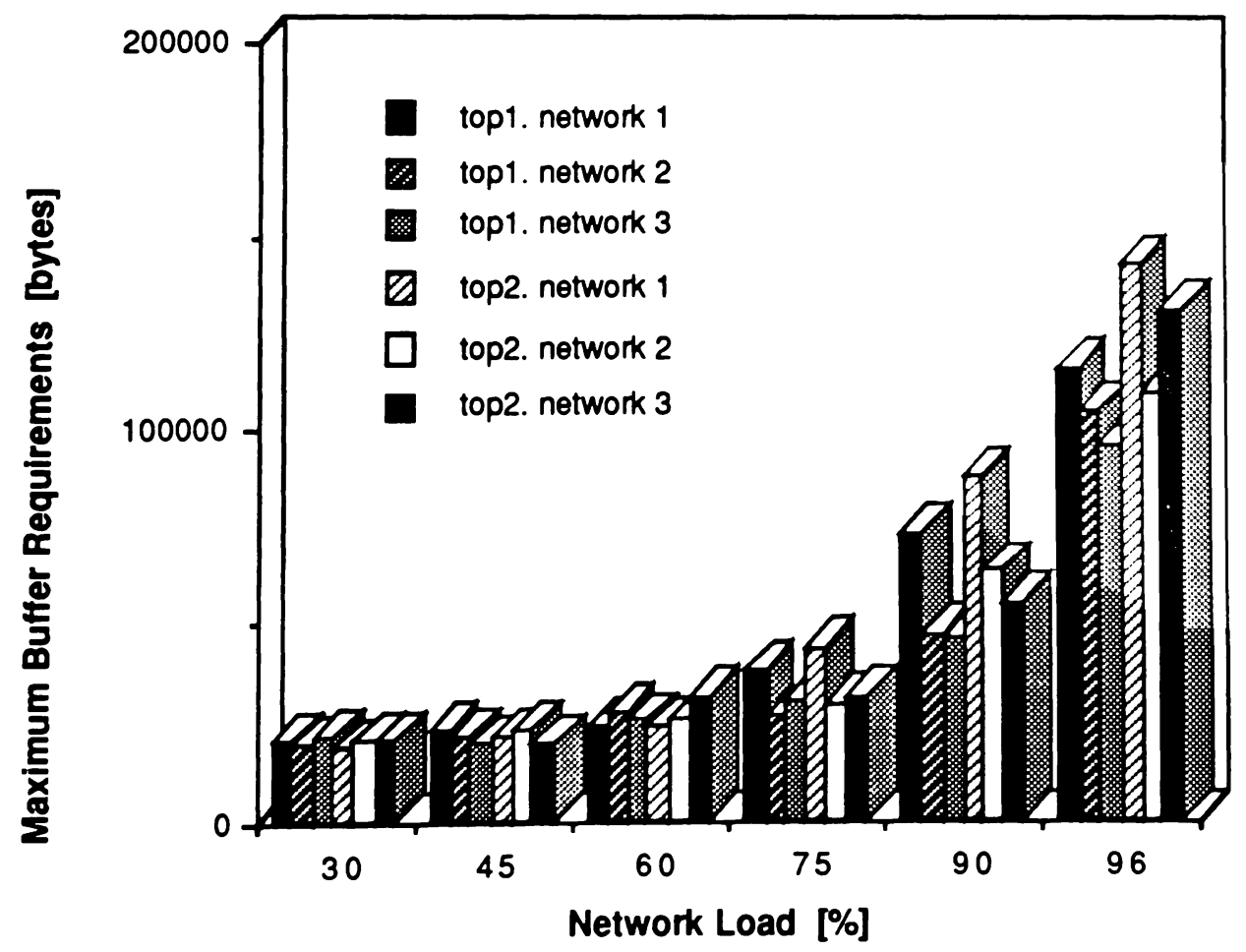

Figure 33. Maximum buffer requirements: topology 1 vs. topology 2 In figure 34 we compare the end-to-end delay for the case of: 
- $\quad$ VC-traffic :

External traffic:

$\underline{\text { IPP }}$

$\underline{\text { MMPP }}$

The parameters for the External MMPPs and VC-IPP are shown in section 5.2.3, table 3 and 4, respectively. Recall that the MMPP and IPP parameters are chosen to be the same in terms of mean arrival rates in Mbps for both topologies. We have also kept the parameters $\alpha$ and $\beta$ the same. However, arrival rates in states 1 and $2, \lambda_{1}$ and $\lambda_{2}$, have to be computed for each topology for the desired total VC-arrival rate in terms of packets/ms. $\lambda_{1}$ and $\lambda_{2}$ values [packets/ms] are going to be different in topologies 1 and 2 since the mean packet length in the two topologies is different (see also discussion in footnote 8). The $c^{2}$ values for the VC-IPP in the topology 1 are constant, equal to 10 , for each simulation point; $c^{2}$ values for the VC-IPP in the topology 2 are different because of the different $\lambda_{1}$ and $\lambda_{2}$ values.

The $c^{2}$ values for the topology 2 VC-IPP turned out to be around 8 except for the second point where the $c^{2}$ value was 18.6. The higher $c^{2}$ value is the reason for the higher packet delay for this point. Hence, the effect of burstiness on the end-to-end delay may be significant, as demonstrated several times so far.

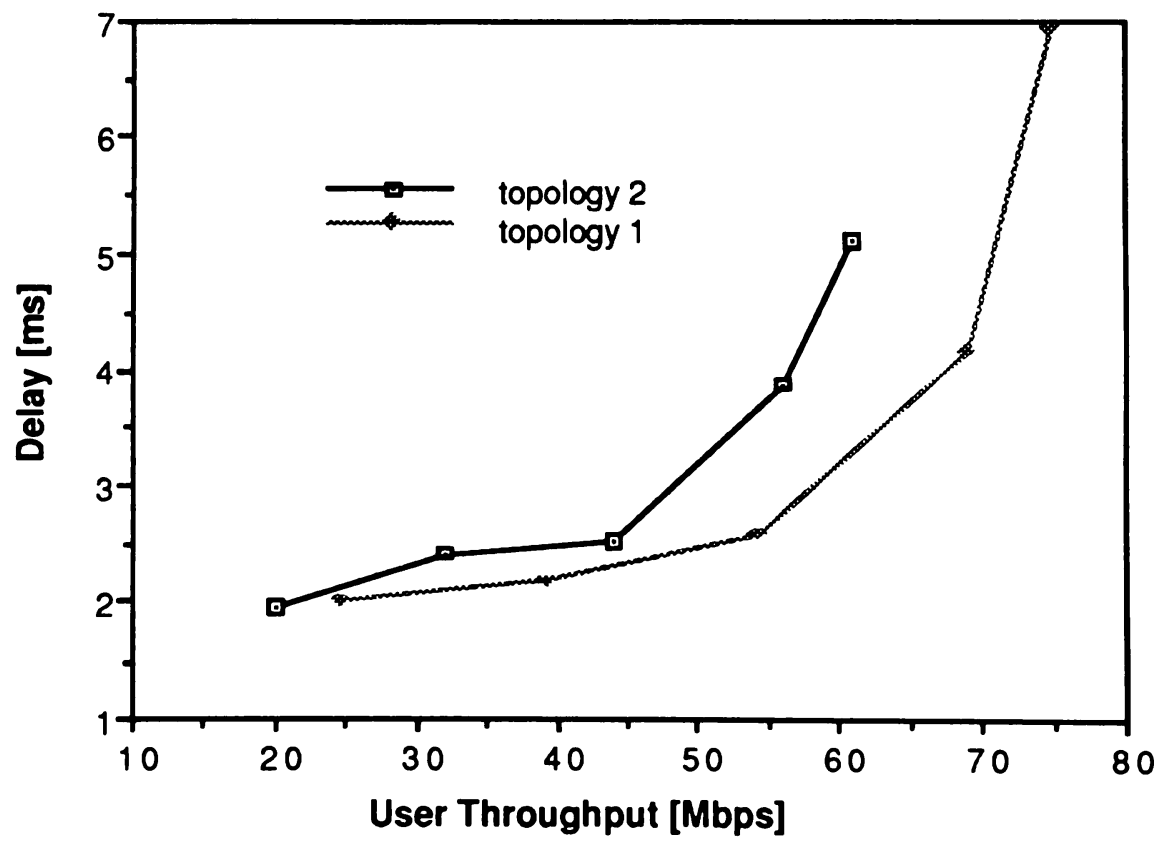

Figure 34. Mean end-to-end delay as a function of user throughput: IPP arrivals, topology 1 vs. topology 2

In figure 35 we show the end-to-end delay for the case: 
- VC-traffic: Poisson External traffic: Poisson 50 Mbps

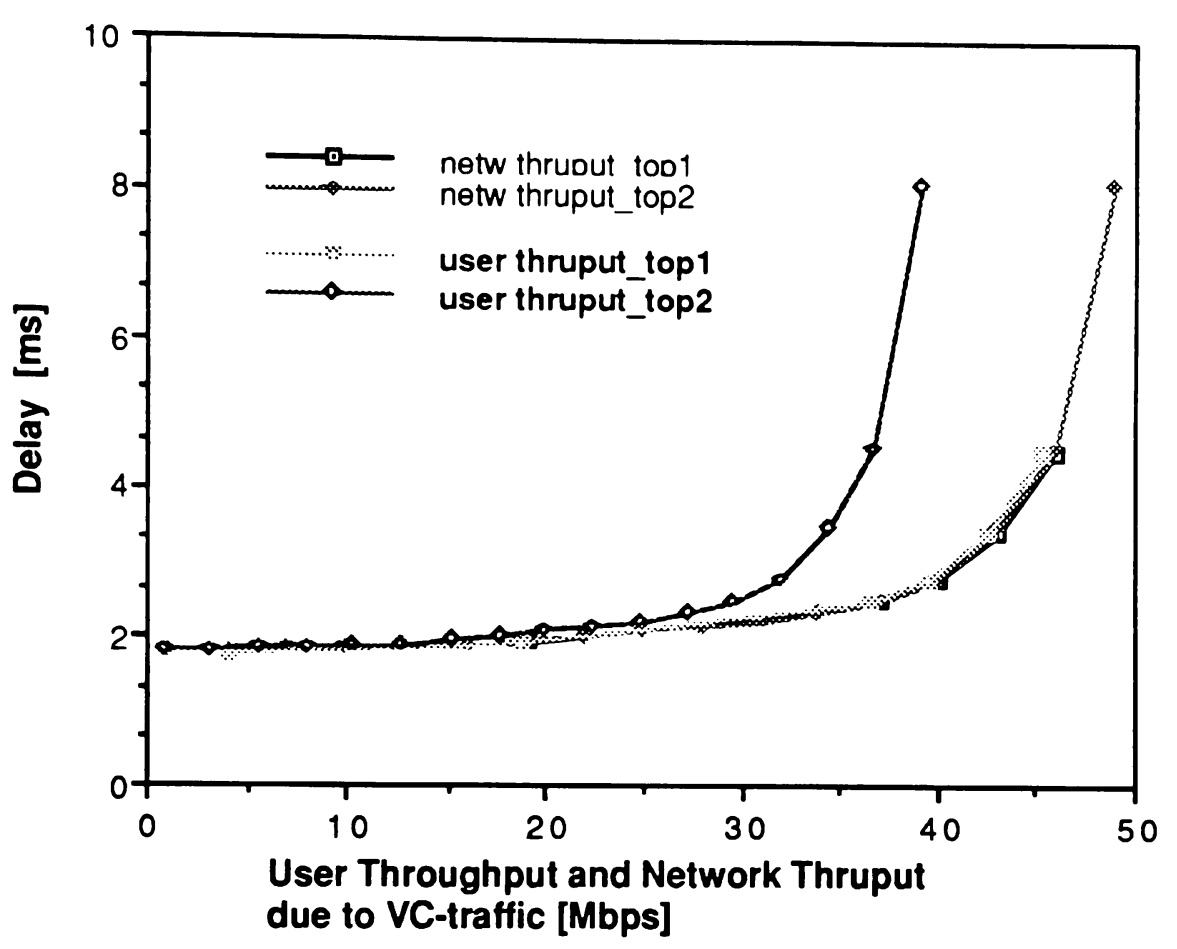

Figure 35. Mean end-to-end delay as a function of user throughput and network load : topology vs. topology2

The curves show clear advantage in the end-to-end delay for the topology in the area of high network utilizations. In the low network utilizations, the delays for both topologies are approximately the same. Recall that the external traffic here is $50 \mathrm{Mbps}$ and packet sizes are 'variable'. The maximum throughput for the user packets in the case of ATM network is $37.5 \mathrm{Mbps}$ due to $25 \%$ overhead, as demonstrated in the upper the graph. 
In figure 35 we show the comparison of the end-to-end delay curves for:

- VC-traffic :

Poisson

pkt.length $=$ const. $(480$

bytes)

Ext. traffic : Poisson50 Mbps pkt.length = 'variable'

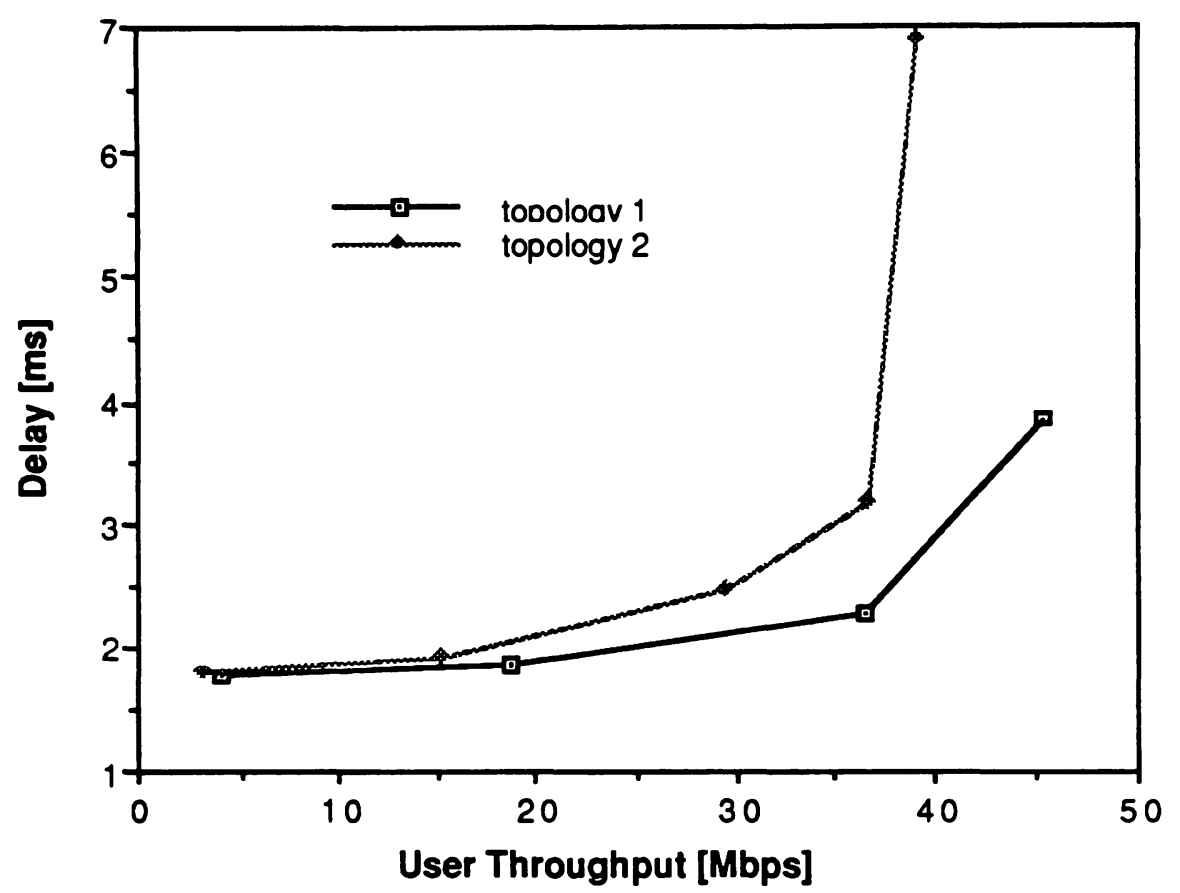

Figure 36. Mean end-to-end delay as a function of user throughput: topology 1 vs. topology 2

In this case we assumed that each user packet ( 480 bytes) is segmented into 10 ATM cells with no padding bits (we ignored the 4 bytes overhead from the Adaptation layer). The average packet length in the case of 'variable' packet size, assumed for the external packets, is 524.7 bytes. Therefore, the assumptions in this case should be beneficial to the ATM approach. The results in the above figure indicate that the end-to-end delay is still larger in the case of ATM networks. The delay is significantly larger in the region of higher network utilizations.

One could argue, here, that our assumptions concerning the adaptation overhead (packet segmentation at source and reassembling at destination) are high. Namely, we assumed $0.05 \mathrm{~ms} /$ packet the segmentation overhead and $0.004 \mathrm{~ms} /$ cell reassembling overhead. If we set both of those parameters to be equal to zero, meaning the ATM adaptation layer does not incur any delay, the above curves would look like those in Figure 37. 


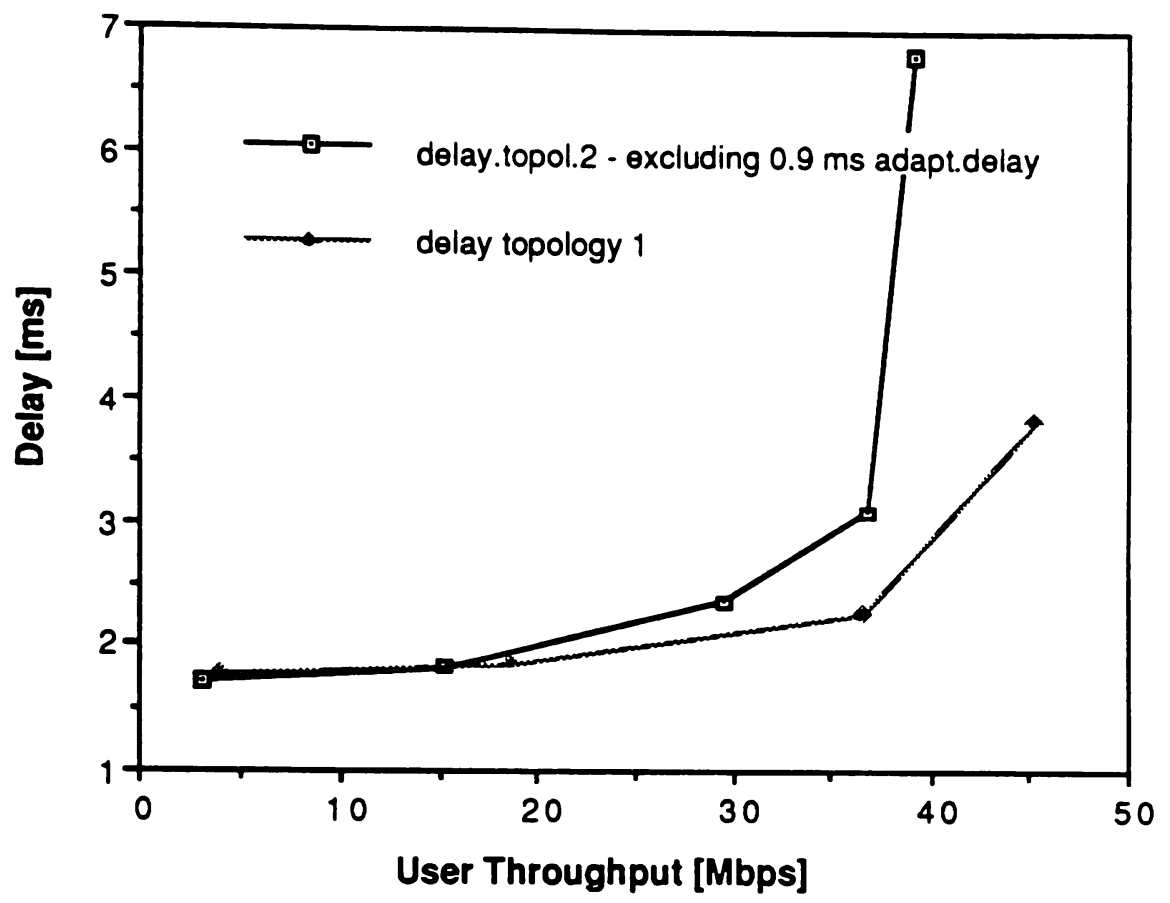

Figure 37. Mean end-to-end delay as a function of user throughput: no ATM adaptation processing delay; topology 1 vs. topology 2

Although the figure shows slight cross-over of the two curves, we can claim that the delays in both topologies are the same in the region of low network utilizations.

We would like to point out that we are considering here three hop end-toend paths. It may be the case that due to the 'pipelining effect' of the ATM cells in topology 2 , the packets in a network with more hops will experience lower end-to-end delays in the region of low utilizations than in topology 1 . We estimate that the advantage to the ATM network in the area of low network utilizations is going to be marginal.

However, it must be pointed out that if there is an advantage in the delay to the ATM network in the case of more hops and low utilizations, it will come at a cost of operating the network under higher utilizations (due to the large overhead in the ATM network, which is in the best case $17 \%$ of the offered load). Operating the network under higher capacity means reducing the available network capacity to the other users.

To demonstrate the above argument we have drawn the mean end-to-end delay with respect to the user throughput for the case of varied external traffic (figures 38 and 39) for both topologies. 

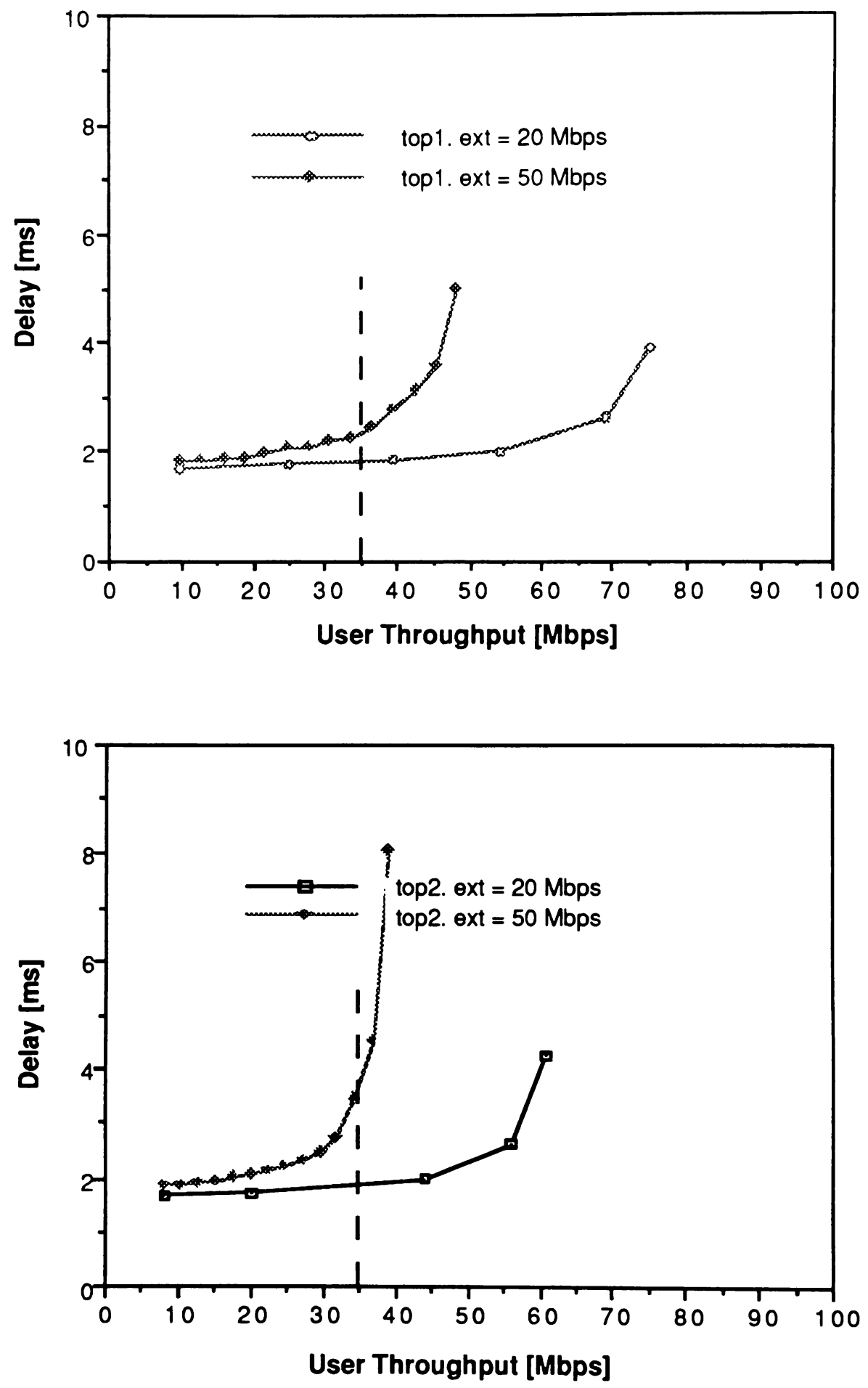

Figure 38 and 39. Mean end-to-end delay vs. user throughput, Ext.-traffic 20 and 50 Mbps: topology 1 (top), topology 2 (bottom)

Let us now take the following example: 
If we assume that the user is requiring the end-to-end throughput of 35 Mbps and the external traffic in both networks is $20 \mathrm{Mbps}$, then the diagram shows that the delays for the two networks is going to be similar, around $1.9 \mathrm{~ms} /$ packet, for both topologies (our results show slightly less packet delay for the Megacell network, but we may neglect this advantage). If the external traffic increases to $50 \mathrm{Mbps}$, the delay in the two networks will be significantly different. The delay on Megacell networks will still be low ( $2.7 \mathrm{~ms} /$ packet) and the delay on ATM networks will be considerably higher, since all three ATM networks are already operating in the congestion region. If the external traffic would increase further, the delay on the ATM networks will be unacceptable. 


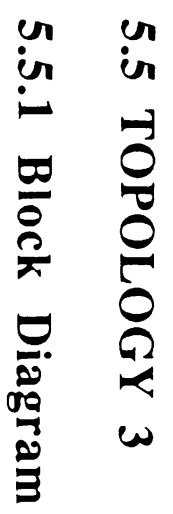

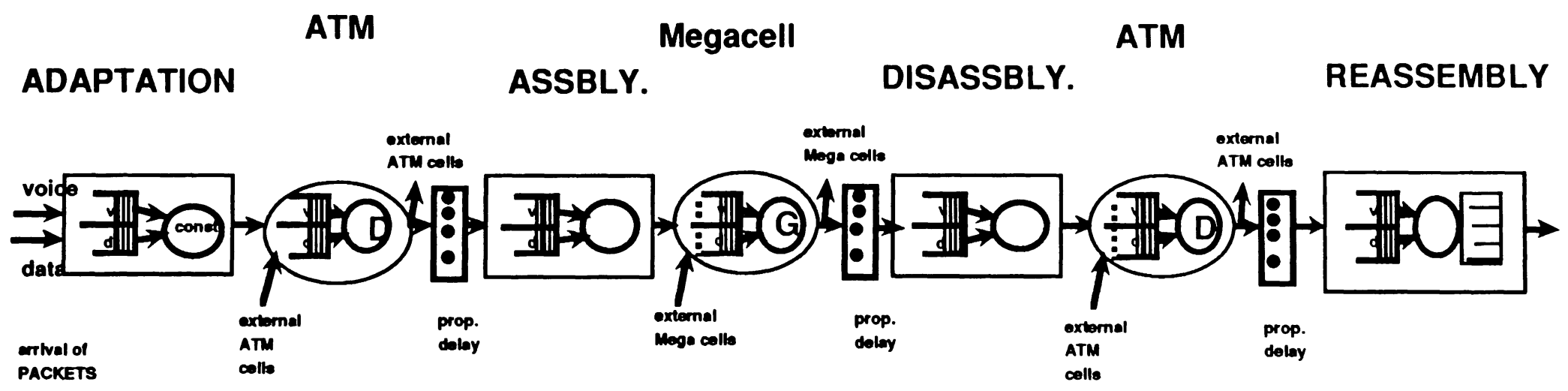




\subsubsection{Discussion of Results}

User packets are segmented into cells and transmitted first through the ATM network. ATM cells arrive, in-turn, to the Megacell network where they may be assembled into larger units of variable size (megacells). Each megacell is appended a 3-10-byte megacell header. Upon leaving the Megacell network, megacell headers are stripped-off and megacells are disassembled into the original ATM cells. In the last hop, ATM cells are transmitted over an ATM network, reassembled into packets at the destination Adaptation layer and delivered to the end-user.

The focus of our studies in this topology is on the 'Assembly' module (see also section 2.4.). Different schemes for assembling ATM may be proposed to optimize different parameters (e.g. throughput of the Megacell network and end-to-end delay for data packets). In this study we are assuming that our goal is to minimize the end-to-end delay for user packets.

If the ATM cells would be transmitted without being assembled into larger units (megacells), the utilization of the Megacell network would considerably increase since each ATM cell would be added a megacell header. Due to the increase in overhead, the end-to-end packet delay would be larger. Therefore, transmitting ATM cells in the Megacell network on a cell by cell basis is obviously not an optimal solution and a more sophisticated approach for handling the incoming ATM cells is necessary.

We present the results for two assembling schemes: a 'Time-Slice' scheme and a 'Fixed Number of ATM Cells' scheme. The 'Hybrid' scheme, mentioned in section 2.4., is a part of our future work.

For the simulations in this topology we have assumed Poisson arrivals for the VC- and External traffic. External arrivals are $50 \mathrm{Mbps}$ and packet lengths are 'variable'. No cell loss is assumed. 


\section{'Fixed Time-Slice' scheme:}

The incoming ATM cells are stored in an 'assembly' queue and a megacell is formed every fixed time period.

In the following example we have assumed:
- Time-Slices:
$0.1 \mathrm{~ms}$
and
$0.05 \mathrm{~ms}$

Figure 40 shows the mean end-to-end packet delay vs. VC-traffic arrival rate for the two time-slices. In the region of high network utilizations, the shorter time-slices are more preferable for the following reason: If the time-slice is long, a large number of ATM cells are waiting in the queue to be assembled into a megacell and eventually transmitted. If user packets are small (consist of a small number of ATM cells), there is a high probability that all the cells from one or more short packets are waiting in the assembly queue. Therefore, the end-to-end delay for shorter user packets becomes larger and, consequently, the overall mean end-to-end delay for user packets increases. On the other hand, if the time-slices are too short, a small number of ATM cells are assembled together and the number of megacell headers transmitted per packet becomes larger. This leads to an increase in the mean end-to-end packet delay.

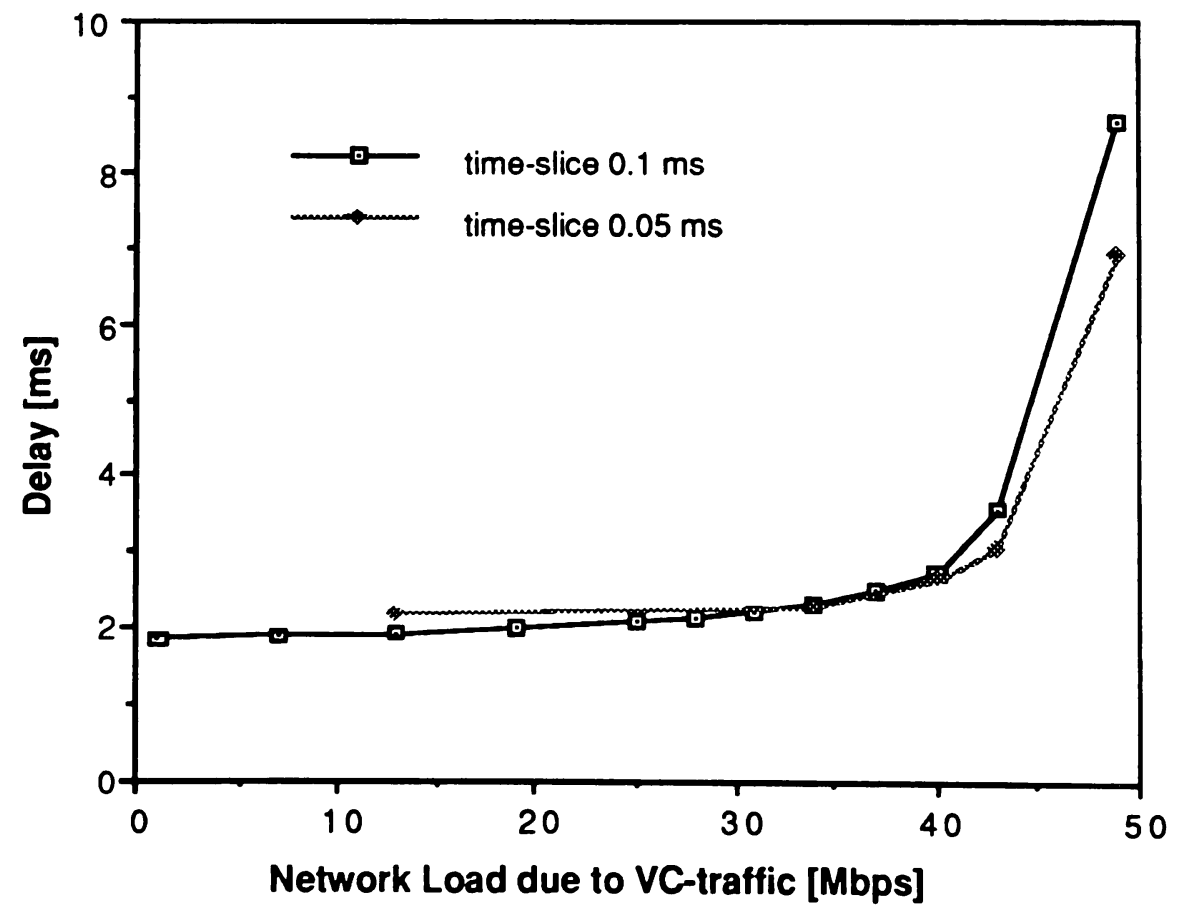

Figure 40. Mean end-to-end delay vs. Network Load due to VC-traffic: 'fixed time-slice' scheme, topology 3 
'Fixed block-size' scheme:

The incoming ATM cells are stored in an 'assembly' queue and a megacell is formed upon arrival of a fixed number of ATM cells (a block-size).

In figure 41 we have assumed:

- Block-sizes: $\quad 5,13,25,100$ ATM cells

Figure 41 shows the mean end-to-end packet delay vs. VC-traffic arrival rate for the above block sizes at the 'assembly'. The discussion of the results in the region of high network utilizations follows a similar argument as in the 'fixed cell-size' case: the arrivals of ATM cells are frequent and it is advantageous to form larger block-sizes (megacells) and, consequently, less overhead transmitted. On the other hand, if network utilizations are low, arrivals are less frequent and the cells that have already arrived into an 'assembly' queue are going to wait for another ATM cells to arrive to form the necessary block-size.

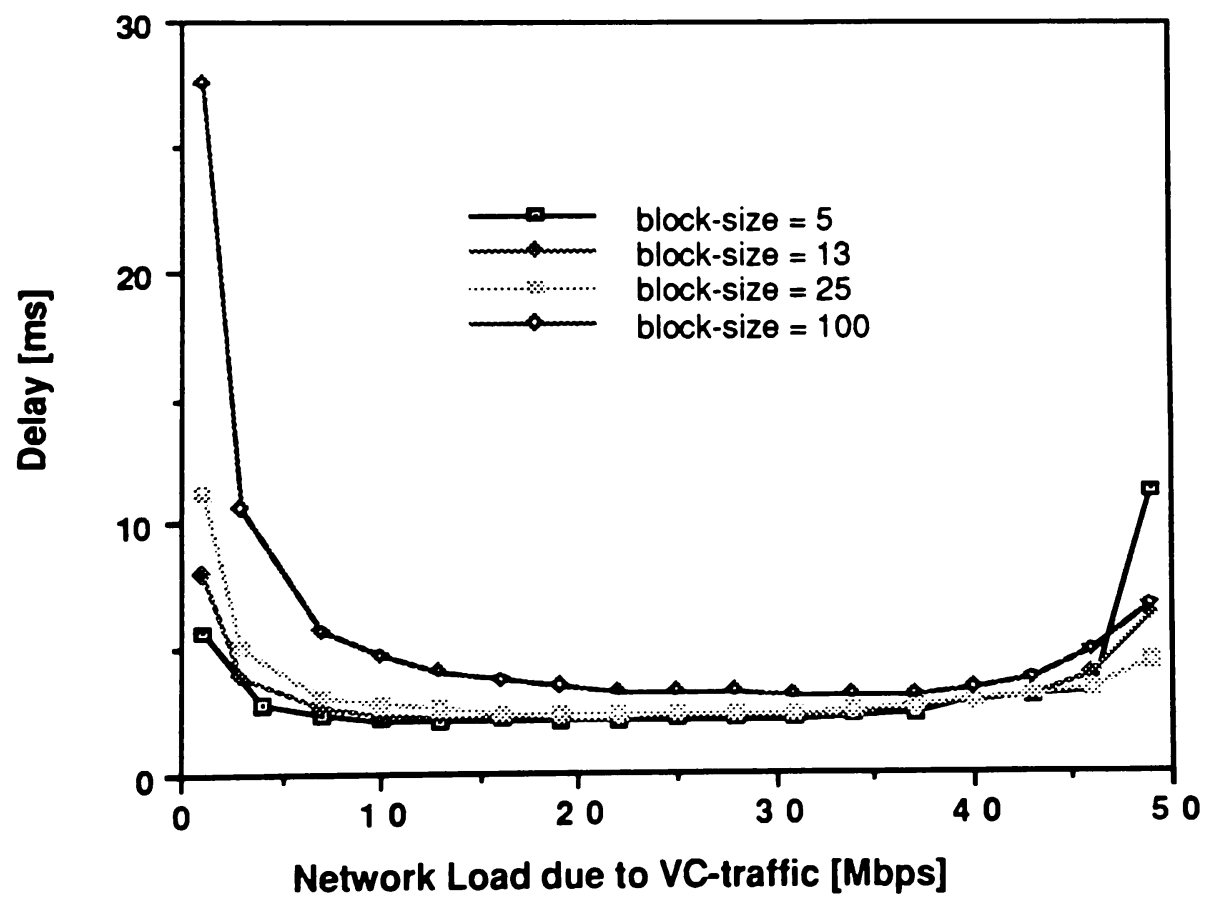

Figure 41. Mean end-to-end delay vs. Network Load due to VC-traffic: 'fixed block-size' scheme, topology 3 
An interesting remaining question is: what is the end-to-end performance in the case that ATM cells are transmitted one-by-one through the Megacell network. Diagram in figure 42 compares the cases of:

\section{- Block-sizes: 1 and 5 ATM cells}

To our surprize, the mean end-to-end delay for the case of block-size $=1$ turned out to be comparable or lower to the delay for the block-size $=5$ ATM cells except in the region of high network utilizations. The curve for block-size $=1$ does not go up in the region of low network utilizations since the ATM cells do not wait in the 'assembly' block for another ATM cells to arrive. The only function 'assembly' block performs is appending the megacell header to the ATM cell. However, one has to keep in mind that if all the ATM cells were transmitted on a Megacell network without being assembling into larger units, the Megacell network load imposed by the VC-traffic would increase $12.23 \%$ (6.5 bytes overhead / 53 bytes ATM cell), assuming megacell header is uniformly distributed between 3-10 bytes. The available transmission capacity of the Megacell network would, therefore, be significantly decreased if large portion of traffic comes from the ATM networks.

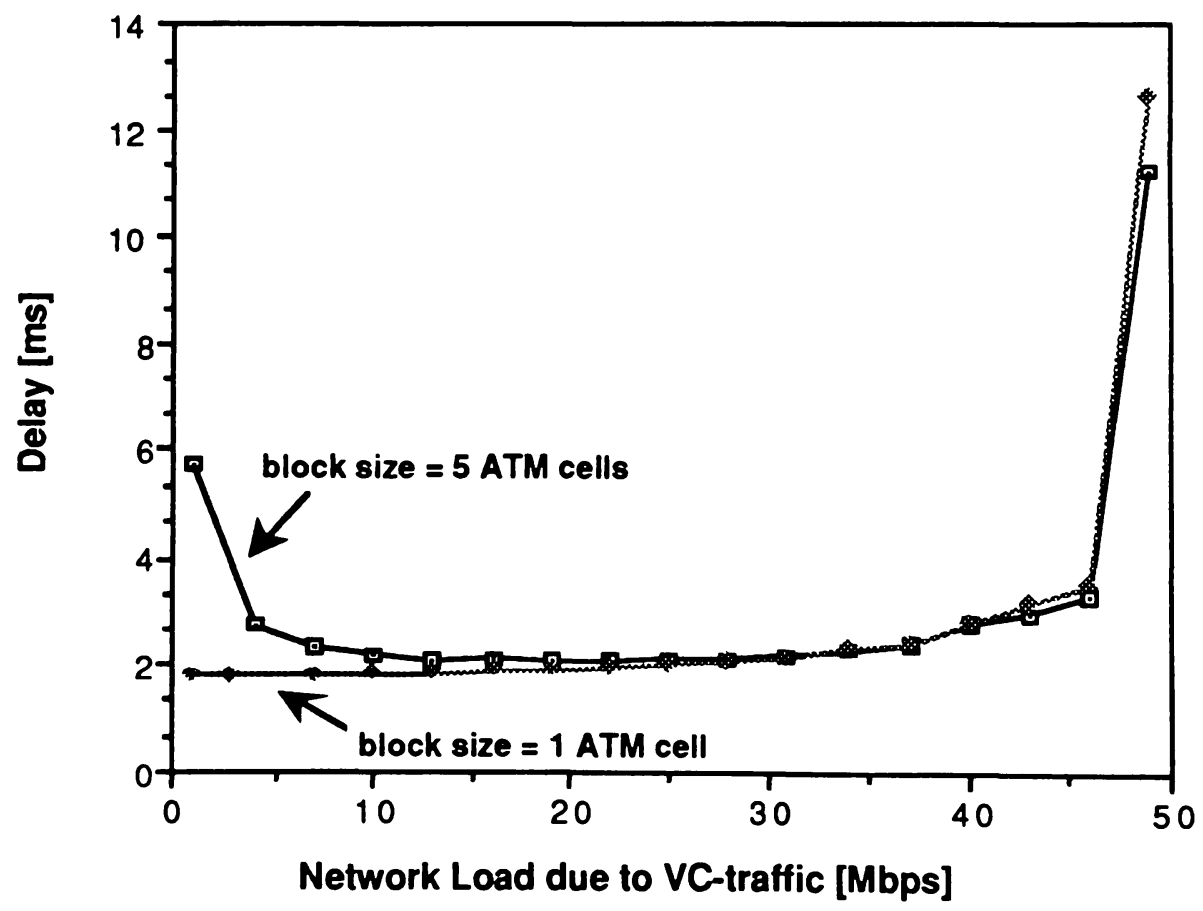

Figure 42. Mean end-to-end delay vs. Network Load due to VC-traffic: 'fixed block-size' scheme, topology 3 
The advantage of a 'Time-Slice' scheme is that the ATM cells will have a guaranteed service every time-slice. Hence, in the periods of a low network utilization the 'Time-Slice' scheme may be better than the 'Block-size' scheme in terms of end-to-end packet delay. On the other hand, in the region of high network utilizations, a large number of ATM cells may be accumulated in the buffer and the average delay for the 'Time-Slice' scheme may be larger.

This shows the trade-off between the two schemes. The 'Hybrid' scheme (see section 2.5) takes advantage of both previous schemes and intuitively yields to the lowest end-to-end packet delay.

The evaluation of the 'Hybrid' scheme is part of our future work. 


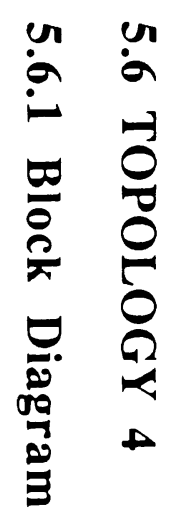

Megacell_1

ATM

Megacell_2

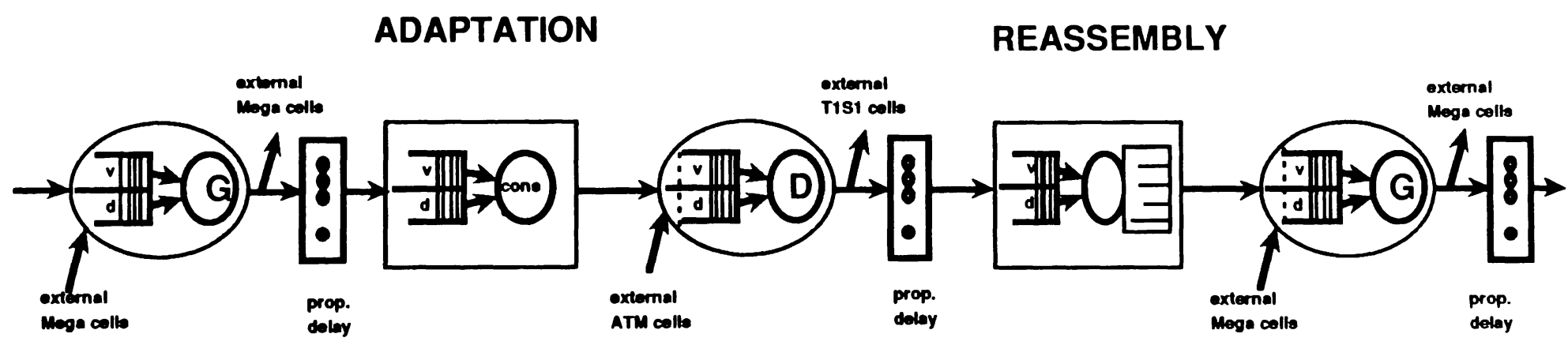




\subsubsection{Discussion of Results}

This topology is similar to topology 1 except that user packets are segmented in the middle network into ATM cells. The Megacell network appends megacell header to the user packets. We assumed that this header is not stripped-off at the exit point of the first Megacell network. Hence, the Adaptation module segments the entire megacells into ATM cells. Upon exiting the network, ATM cells are reassembled back into megacells and sent through the second Megacell network to the destination. The megacell header is now stripped-off and the packets are delivered to the end-user.

We show only one simulation result for this topology. Poisson arrivals are assumed for the VC- and External traffic. External arrivals are $50 \mathrm{Mbps}$. No cell loss is assumed. Packet lengths are 'variable'.

Figure 43 shows that the maximum user throughput in this case is around $43 \mathrm{Mbps}$. The reason for this is that the 'middle' network is an ATM network and to transmit packets across an ATM network a considerable amount of overhead has to be transmitted (in this case packet length distribution is 'variable' and in this example we assumed that user packets are segmented into 48-byte chunks instead of 44 bytes), overhead accounts for $16.7 \%$ of the additional network load. Hence, if the user throughput is $43 \mathrm{Mbps}$ and external loading is $50 \mathrm{Mbps}$, the ATM network throughput is going to be $50 \mathrm{Mbps}$, which means that the ATM network is running at 100 $\%$ utilization.

This example shows clearly the large impact of the ATM overhead on the end-to-end throughput for user packets.

The results for the bursty arrivals for this topology are part of our future work. 


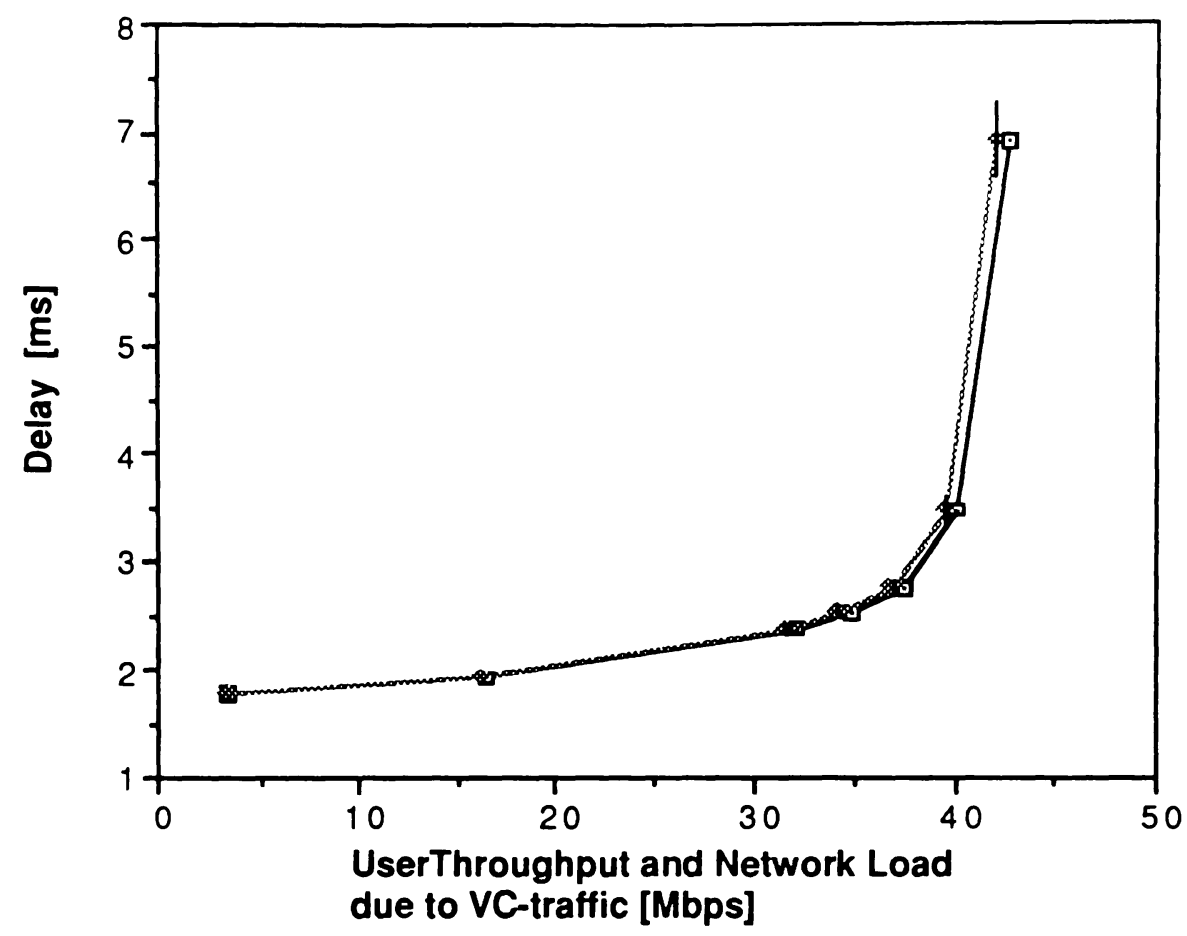

Figure 43. Mean end-to-end delay vs. User Throughput and Network Load due to VC-traffic: topology 4 


\subsection{TOPOLOGY 1 - 4 : A COMPARISON}

In figure 44 we show the end-to-end delay results for the four topologies with the following assumptions:

- $\quad$ VC-traffic:

External traffic:

Network speeds:

packet length:
Poisson

Poisson, $50 \mathrm{Mbps}$

$100 \mathrm{Mbps}$

'variable'21

Further, we assume that user packets are segmented into 48- instead of 44byte chunks 22 in the Adaptation module. Hence, in the case of the 'variable' packet length distribution, additional load due to the overhead on an ATM network is $16.7 \%$. As a representative for the topology 3 , we selected results from the $0.1 \mathrm{~ms}$ 'time-slice' case. No cell loss is assumed.

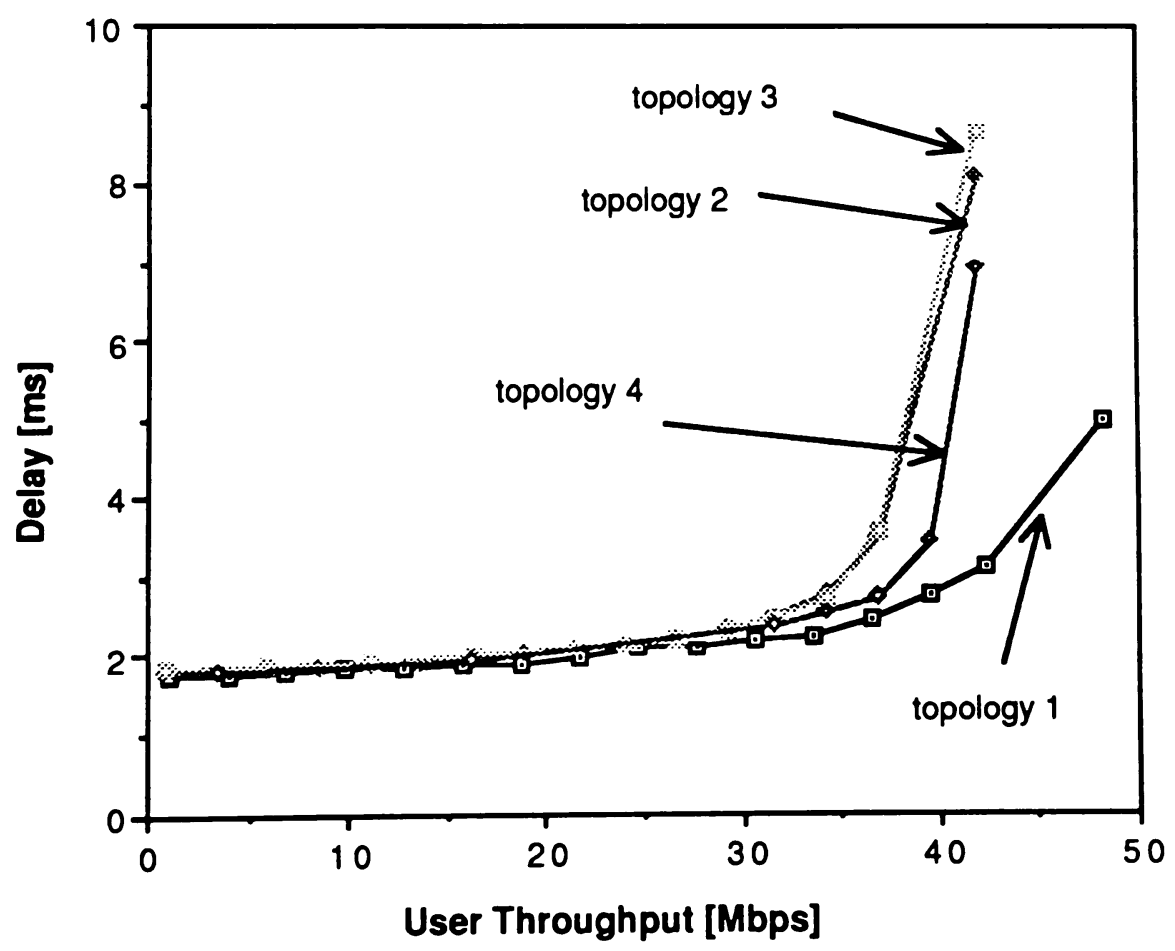

Figure 44. Mean end-to-end delay: Topology 1-4

21 The 'variable' packet length distribution is shown in table 2 or section 3.7, figure 5

22 The above curves were obtained early in the project, where we initially assumed a 5 byte overhead and 48 byte information field in the ATM cell 
Given that the external load at each network in each topology is $50 \mathrm{Mbps}$ and the 48-byte ATM cells are assumed, the maximum throughput in any topology including an ATM network is $42.84 \mathrm{Mbps}^{23}$ Hence, only topology 1 achieves throughput close to $50 \mathrm{Mbps}^{24}$ (49.28 Mbps). The results would, of course, be much more in favor to the Megacell network if the ATM cell was assumed with 9 bytes of overhead and 44 bytes of data (this we assumed in the majority of the studies presented in this report). Namely, the maximum throughput for the topologies 2,3,4 (due to ATM networks) would drop to $40 \mathrm{Mbps}^{25}$.

The performance for the topologies 2 and 3 is very close ${ }^{26}$ for almost the entire delay curve. This is due to the fact that the introduced time-overhead due to the Assembly module in topology 3 is not significant because the selected 'time-slice' of $0.1 \mathrm{~ms}$ does not delay the incoming cells much. At the higher utilizations, ATM cells accumulate in the Assembly queue and the delay increases ${ }^{27}$.

Since topology 4 includes an ATM network in the end-to-end path, the maximum user throughput in the above case is $42.84 \mathrm{Mbps}$. Comparing now topology 4 against topology 2 , the end-to-end packet delay is approximately the same in the region of low and moderate network utilizations. In the area of high network utilizations, topology 4 offers lower delays for the same user throughput. In the high network utilization region the delay increases significantly for an incremental additional load imposed. However, the delay for topology 4 starts increasing for higher user throughputs than the delay for topology 2 because the additional load caused by the ATM network overhead needs to be transmitted only at the 'middle' network (ATM) and the utilization of the two other Megacell networks remains low enough for the delay to be increased by a large amount.

23 As explained in the discussion of the results for the topology 4 (section 5.6.2), the 50 Mbps maximum network load from the VC-traffic is divided by the expansion factor for the ATM network, here 1.167, to obtain the maximum user throughput

24 The expansion factor for the Megacell network is in the case of the 'variable' packet length distribution 1.0145

25 The expansion factor for the ATM network in this case is 1.25

26 The results for the topology 3 are slightly higher, due to the waiting time for the ATM cells in the Assembly queue and also because the Assembly module appends a Megacell header for every group of the assembled ATM cells accummulated during $0.1 \mathrm{~ms}$ 27 At $49 \mathrm{Mbps}$ total VC-network load approx. 11.6 ATM cells arrive on average to the Assembly module within $0.1 \mathrm{~ms}$. Therefore, ATM cells have to wait until they get served. On the other hand, at 1 Mbps total VC-network load approx. 0.235 ATM cells arrive on average to the Assembly module within $0.1 \mathrm{~ms}$. Hence, waiting time per ATM cell once it is in the queue is low. 


\section{CONCLUSIONS}

In this report we have performed an extensive study of ATM and Variable Length Cell size networks and their interconnections for the various assumptions on packet lengths and inter-arrival time distributions. The scope of this project is very broad and we have not been able to give equal attention to each problem and each topology. Hence, as we mentioned earlier, we had to make some constraints with respect to model assumptions and focus on issues for which we believed that will contribute best to the desired comparisons.

The studies were done by simulation and were not compared against the analytical results. This makes it somewhat predated to draw firm conclusions about certain comparisons. However, given the underlying model assumptions, from the results presented in this report we can make the following conclusions:

\section{ATM vs. Variable Length Cell (Megacell) networks}

To transmit the same amount of data on an ATM and Megacell network, the ATM network will have to transmit an additional load of at least $16.9 \%$ more information due to the large overhead ( 9 bytes) in each ATM cell. If we assume that the capacities of the two networks are the same, the ATM network will be able to handle fewer end-to-end connections.

For a three hop network, the end-to-end delay is lower in the case of Megacell networks. The difference in delay in the region of low network utilizations is almost negligible. However, in the region of medium and high network utilizations, delay in the ATM network is significantly higher. Also, if the two networks have the same transmission speeds, it is impossible to compare the two networks for the same user throughputs in the region of high network utilizations. While the ATM network reaches the saturation point, the Megacell network serves the same amount of user traffic without a drastic increase in delay.

\section{Assembly module (topology 3 )}

We have shown the performance results for the two schemes for assembling ATM cells at the entry point to the Megacell network (the 'fixed time-slice' scheme and the 'fixed block-size' scheme). Both schemes are simple to implement and have advantages and disadvantages. The 'hybrid' scheme, which has not been implemented yet, will take advantages 
of each of the previous two schemes and presumably lead to the best endto-end delay results. The drawback of this scheme is the increased complexity, particularly in the case when the network will carry traffic of different priority levels.

\section{Effect of burstiness on the end-to-end delay}

We have shown in numerous studies that the effect of burstiness on the endto-end delay is significant. Modeling broadband networks with different types of traffic (voice, video, data) by assuming the Poisson arrivals is not permissible and leads to false predictions concerning the end-to-end delay and cell loss probability. Performance analysis of the computer networks with bursty arrivals and correlated inter-arrival times is a fairly new area which deserves further attention.

Although the effort which was put in generating the simulation results and comparisons for the topologies 1-4 has been extensive, there are still a number of unresolved issues and possibilities for further model improvements. We discuss these issues briefly in the next chapter.

\section{Main contributions:}

Our contribution to the published research in terms of modeling ATM networks can be summarized as follows:

- We have performed an end-to-end delay study for the packets on a particular Virtual Circuit Connection on Fixed Length Cell-size (ATM), Variable Length Cell-size and the mixed type interconnected networks

- We have compared network performance under Poisson and MMPP arrival processes. MMPP allows capturing of burstiness and high correlation of inter-arrival times, which are expected in BISDN

- We have performed end-to-end study by allowing external traffic to be mixed with the VC-traffic, thereby providing an opportunity to examine end-to-end delay for a particular application under varied network conditions

- We are tackling the problems of optimal packetization of information when interfacing different cell size networks 


\section{FUTURE WORK}

In the current models we have assumed batched arrivals for the external traffic in the case of ATM networks. In general, it is very difficult to say what is the correct assumption for the external arrivals to the ATM networks and the assumption we made may be good. However, if the batch cell arrivals are assumed for the external ATM traffic, then the cells from the VC-traffic are not able to interleave between cells belonging to the external traffic. On the other hand, if a long external packet arrives (batch of ATM cells) during the arrival of VC-cell traffic, it will be interleaved between the VC-cells in the ATM queue and the waiting time for the later VC-cells will be higher. This gives an advantage to the external cells and may lead to a higher end-to-end delay performance of the VC-traffic.

One possible way to approach this problem can be, for example, to measure the inter-departure times and burstiness of departures after the three (or more) ATM networks and then use the obtained parameters to match the external arrival process. A departure process obtained after several networks should be statistically equal to the desired external arrival process due to the fact that the external traffic is assumed to be an aggregate network traffic which has traversed several network hops prior to interfering with the VC-traffic. A more elegant way of solving this problem would be to obtain the desired departure distribution characteristics analytically.

Models would also be improved by introducing priorities and obtaining results per priority class. However, the simulation and particularly the analytical analysis is much more difficult.

For the comparison of ATM and variable length cell-size networks it may be useful to investigate the end-to-end delay in the two networks for more than three intermediate hops. As we pointed out earlier, it is to be expected that ATM network with more intermediate hops will have lower end-toend delay in the region of low network utilizations. The difference in the delay should not be significant. This could be investigated by new simulations with an increased number of hops in our models or, more beneficially, through an analytical solution.

We believe that after these extensive simulation studies we have gained a good insight into the end-to-end delay performance of the four topologies and should at this point switch our attention to analytical models. 


\section{REFERENCES}

[1] CCITT I. 121 and I.150, Recommendations drafted by Working Party XVIII / 8 (General Broadband ISDN Aspects) to be approved in 1990, June 1990.

[2] ANSI X3.148, Fiber Distributed Data Interface (FDDI) - Token Ring Physical Layer Protocol (PHY), June 1988.

[3] ANSI X3T9REV3, FDDI MEDIA ACCESS CONTROL (MAC-2), Feb. 91990.

[4] ANSI X3T9.5 REV6, FDDI STATION MANAGEMENT (SMT), Jan. 201990.

[5] S. Parekh and K. Sohraby, "Some Performance Trade-Offs Associated With ATM Fixed-Length vs. Variable-Length Cell Formats," in Proc. IEEE Int'l Conf. on Comm., Nov 1988, pp. 1282-1287.

[6] J. Salahi, "Modeling the Performance of a Broadband Network Using Various ATM Cell Formats," in Proc. IEEE Int'l Conf. on Comm., Boston ICC, Boston MA, 11-14 June 89,, 1989, pp. 1208-1212.

[7] I. Cidon et al., "ATM Fast Packet Switching: A critique of ATM from a data communications perspective," IBM, March 81990.

[8] A. DeSimone and V.R. Saksena, "Performance of Alternative MAN Architectures For High-Speed Data Applications," in Proc. International Conference on Communications (ICC), 1990, pp. 1584-1590.

[9] D.R. Cox and P.A.W. Lewes, "The Statistical Analysis of Series of Events," Methuen and Co. Ltd.,London: 1968.

[10] A.E. Eckberg, "Generalized Peakedness of Teletraffic Processes," in Proc. 10'th International Teletraffic Congress, Montreal, 1983.

[11] A.E. Eckberg, "Approximations for Bursty (and smoothed) Arrival Queueing Delays Based on Generalized Peakedness," in Proc. 11 'th International Teletraffic Congress, Kyoto, 1985.

[12] W. Whitt, "The Queueing Network Analyzer," Bell Systems Technical Journal, vol. 62, pp. 2779-2815, 1983.

[13] D. Feldmeier, "Traffic Measurements on a Token Ring Network," in Proc. Computer Networking Symp., Washington DC, Nov. 17-18, 1986, pp. 236-243.

[14] P. Amer et al., "Local Area Broadcast Network Measurement: Traffic Characterisation," in Proc. IEEE Computer Society International Conference 32nd, Piscataway, NJ, 1987.

[15] R. Gusella, "Analysis of Diskless Workstation Traffic on An Ethernet," Technical report: AD-A196100 / 2/XAB, pp.27.

[16] P.J. Kuehn, "Approximate analysis of general queueing networks by decomposition," IEEE Transactions on Communications, vol. COM-27, pp. 113126, 1979. 
[17] W. Whitt, "Approximating a Point Process by a Renewal Process: Two Basic Methods," Operations Research, vol. 30, no.1, Jan-Feb, pp. 125-147, 1982.

[18] S.L. Albin, "On Poisson approximations for superposition arrival process in queues," Management Science, vol. 28, pp. 126-137, 1982.

[19] S.L. Albin, "Approximating a point process by a renewal process, II: superposition arrival processes to queues," Operations Research, vol. 32, pp. 1133-1162, 1984.

[20] G.F. Newell, "Approximations for superposition arrival process in queues," Management Science, vol. 30, pp. 623-632, 1984.

[21] K. Sriram and W. Whitt, "Characterizing Superposition Arrival Process in Packet Multiplexers for Voice and Data," IEEE, Journal on Selected Areas in Communications, vol. SAC-4, no. No. 6, Sep., pp. 833-846, 1986.

[22] H. Heffes and D. Lucantoni, "A Markov Modulated Characterisation of Packetized Voice and Data Traffic and Related Multiplexer Performance," IEEE J. SAC-4, vol. no. pp. 856-868, 1986.

[23] H. Perros and R. Onvural, "On the Superposition of Arrival Processes for Voice and Data," in Proc. Proc. Fourth Int. Conf. on Data Communication Systems and their Performance, Barcelona, 1990, pp. 341-357.

[24] I. Norros et al., "The Superposition of Variable Bit Rate Sources in an ATM Multiplexer," to be published in Journal of Selected Areas in Communications, April 1991.

[25] J.N. Daigle and J.D. Langford, "Models for analysis of packet voice communications systems," IEEE J.SAC -4, pp. 847-855, 1986.

[26] H. Perros, "Performance Issues in ATM Networks", Center for Communications and Signal Processing and the department of Computer Science, North Carolina State University, January 1990.

[27] M. Véran, B. Sems, and D. Potier, "QNAP2: A portable environment for queueing systems modeling," Reference Manual, 1982.

[28] R. Ballart and Y.-C. Ching, "Now it's the Standard Optical Network," in IEEE Communications Magazine, 1989.

[29] R.J. Boehm, "Progress in Standardization of SONET," in IEEE LCS Magazine, 1990, pp. 8-16.

[30] IEEE 802.6, Proposed Standard: Distributed Queue Dual Bus Metropolitan Area Network, Nov.15 1988.

[31] Daley, "The correlation structure of the output process of some single server queueing systems", The Annals of Mathematical Statistics, vol.39, no.3, 1968, pp 1007-1019. 


\section{APPENDIX A}

The CCITT Broadband-ISDN Standard:

ATM and Adaptation Laver

The CCITT Standard [1] specifies the B-ISDN protocol reference model, as shown in the figure A1.1.

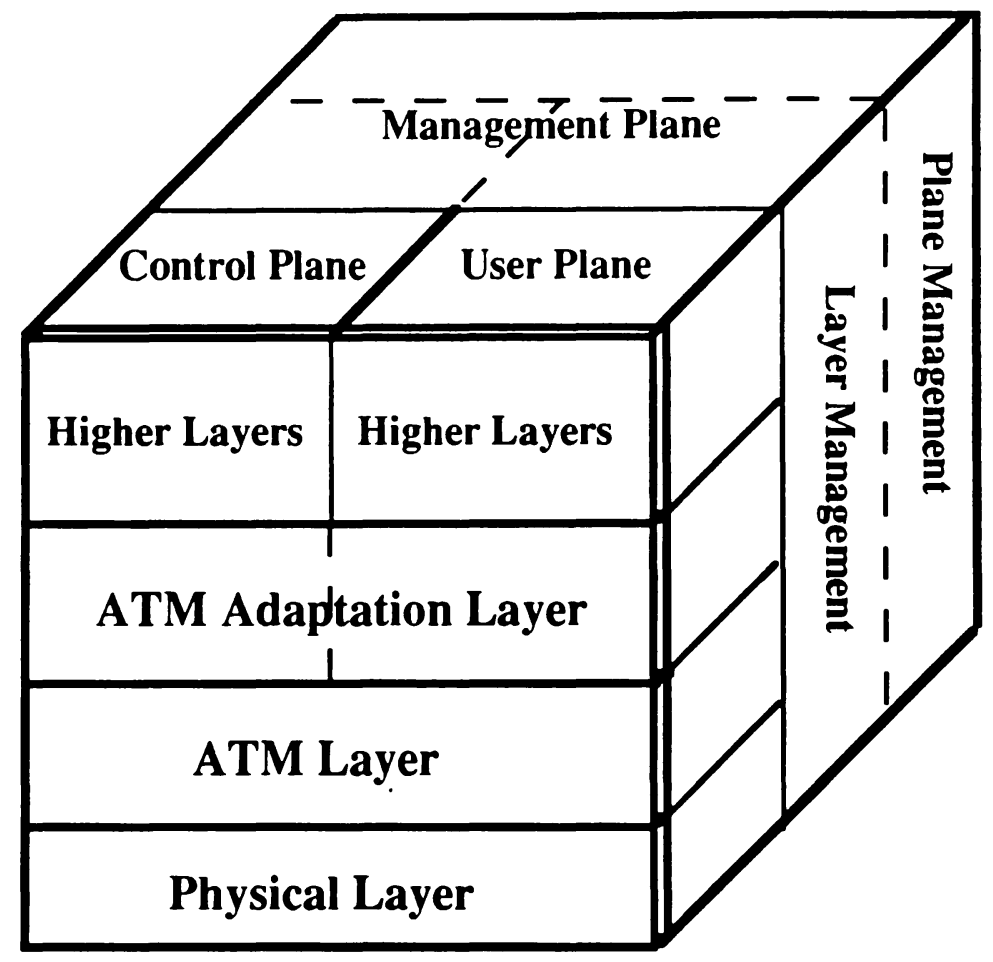

figure A1.1. B-ISDN protocol reference model

The protocol reference model consists of a user plane, a control plane and a management plane. The user plane, with its layered structure, provides for the user information flow transfer along with the associated controls (e.g. flow control, error recovery etc.). The control plane has also a layered structure and performs the call control and connection control functions (e.g. signalling necessary to set up supervise and release calls and connections). The management plane provides two types of functions, the Layer Management and the Plane Management functions. The Plane Management performs management functions related to the whole system and deals with coordination between all the planes. The Layer Management 
deals with the operation and maintenance information flows specific to each layer.

The specification of the physical layer is not in the scope of this standard document. The Physical Layer can be virtually any kind of high speed carrier capable of transporting ATM cells, such as the SONET (Synchronous Optical Network) [28] [29].

In this study we focus solely on the end-to-end delay performance for the user packets at the Adaptation and ATM Layer. We assume the Physical Layer as being transparent and also neglect the effects of the transmission overhead resulting from the control and management plane on the end-toend delay of the user packets.

\section{ATM Laver}

ATM is a specific packet oriented transfer mode using an asynchronous time division multiplexing technique. The multiplexed information flow is organized in fixed size blocks, called cells. CCITT standard specifies ATM cell size to be 53 bytes long. The ATM Layer is responsible for the call transfer of all the services in the ATM network.

ATM is a connection oriented technique. During the 'connection establishment' phase between the source and the destination node, the entries in the routing tables at all intermediate nodes on the end-to-end path are established. Following this, source and destination can exchange information on the established path. The routing information in each ATM cell header is contained in the Virtual Channel Identifier (VCI) and Virtual Path Identifier (VPI) fields. In the ATM switches, the value of the VPI and/or VCI of each incoming ATM cell is mapped into new VPI and/or VCI values of the outgoing VC and VP links. This mapping function could also be null.

\section{- ATM Cell Header:}

The header in the ATM cell is five bytes long. It contains information concerning flow control, routing, type of payload, priority indicator or quality of service, and error detection and correction field for the header. The remaining 48 bytes are available as a nominal payload.

VCI and VPI explicitly identify the Virtual Channel to which the cell belongs to. The type of payload field is used to distinguish between the user 
and the network information carried in the ATM cell information field. The priority indicator bit denotes whether the cell is subject to discard, depending on the network conditions.

It is interesting to point out that the IEEE802.6 draft standard for Metropolitan Area Networks (MAN) [30] employs the same cell structure as BISDN.

\section{ATM Adaptation Laver (AAL)}

The ATM Adaptation layer (AAL) performs functions required by the user, control and management planes and supports the mapping between the ATM Layer and the next higher layer. The functions performed in the AAL depend upon the higher layer requirements. The AAL exchanges with the ATM layer information in a 48 octet ATM - Service Data Units. AAL is divided into two sublayers: the Convergence Sublayer (CS), which interfaces directly with the higher layer; and the Segmentation and Reassembly sublayer (SAR), which is located on top of the ATM layer.

The AAL supports multiple protocols to fit the needs of the different service users. The CCITT standard identifies 4 types of AAL service users:

type 1 - transfer of service data units with a Constant source Bit Rate (CBR)

type 2 - transfer of service data units with a Variable source Bit Rate (VBR),

type 3 - transport of a single AAL Service Data Unit (AAL-SDU) or multiple AAL-SDU in one or more CS Protocol Data Units (CS-PDUs), and,

type 4 - transfer of AAL-SDU to one or more users through the ATM network.

The AAL overhead is different in the size and the type of the header information in the four AAL types. The header size varies from 1 byte for the type $1 \mathrm{AAL}$ to four bytes in the case of type 3 and type $4 \mathrm{AAL}$. Thus, the amount of the available payload to the user per ATM cell is reduced to 44 bytes in the case of a type 3 and type 4 AAL.

In this simulation study we have assumed that the user information are variable size data packets and have focused on a single end-to-end Virtual 
Circuit Connection (VCC). Therefore, the type 3 AAL is assumed with four byte AAL overhead in the study.

Figure A1.2 shows the PDUs at the User, AAL and ATM layer for the case of a type 3 AAL.

User Layer
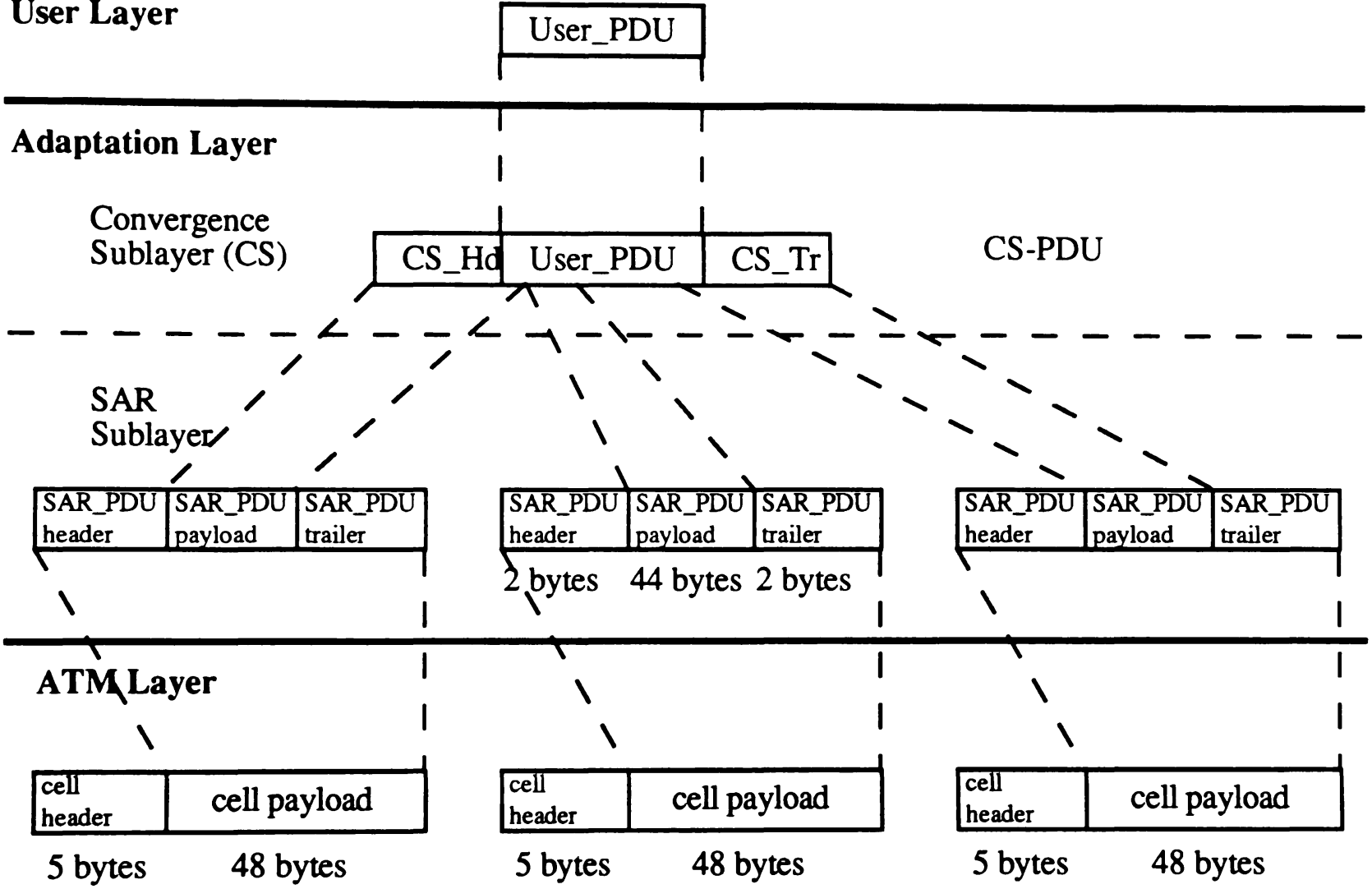

figure A1.2. User, Adaptation and ATM Layer PDUs

- SAR-PDU Header and Trailer (type $3 \mathrm{AAL}$ ):

Since user packets are segmented into cells at this layer, in order to enable reassembling of the cells into packets at the destination a sequence number is necessary in each cell. Moreover, due to the fact that the cells from one packet can be interleaved by the cells from other packets by the sender (e.g. multiplexing of multiple user sessions on a single ATM layer connection), a multiplexing identification field is required. The above two identifiers are located in the SAR-header. The Cyclic Redundancy Checking (CRC) bits, which protect the information octets, are contained in the trailer in each cell. The cell trailer contains also a field providing 
information about the number of octets from the CS-PDU that are included in the SAR-PDU. This is necessary due to the fact that user packets are segmented into an integral number of ATM cells. Therefore, the last ATM cell may not be completely filled with the information octets.

\section{- Convergence Sublayer:}

The CS-sublayer, for which specifications are not yet available in the standard, is expected to perform functions such as passing information to the destination about the total length of the user packet (encapsulated in a CS-PDU) and requesting certain amount of memory to be allocated at the destination for accepting cells of the particular user packet. Since all the cells on the Virtual Circuit Connection follow the same path, the detection of the lost cell is possible from the information about the total length of a CS-PDU and the number of cells arrived at destination. Hence, in the case that the cells get lost, the adaptation layer at the destination may request retransmission of the entire packet from the source.

It is worth noting at this point that the standard document [1] provides two types of operational procedures:

- Assured operations: Every assured AAL-SDU is delivered with exactly the data content that the user sent. The assured service is provided by retransmission of missing or corrupted CS-PDUs.

- Non-assured operations: Integral AAL-SDU may be lost or corrupted. Lost and corrupted AAL-SDU will not be corrected by retransmission. An optional feature may be provided to allow corrupted AAL-SDUs to be delivered to the user. 


\section{APPENDIX B}

Selected results from the 'Study of burstiness of the departure process for the superposition of various arrival process and packet-length distributions'

This simulation study was started in order to gain more understanding into 'burstiness' of the network traffic. The results presented here are restricted to a single node network. The intention is to expand the study in the future for a multiple node network.
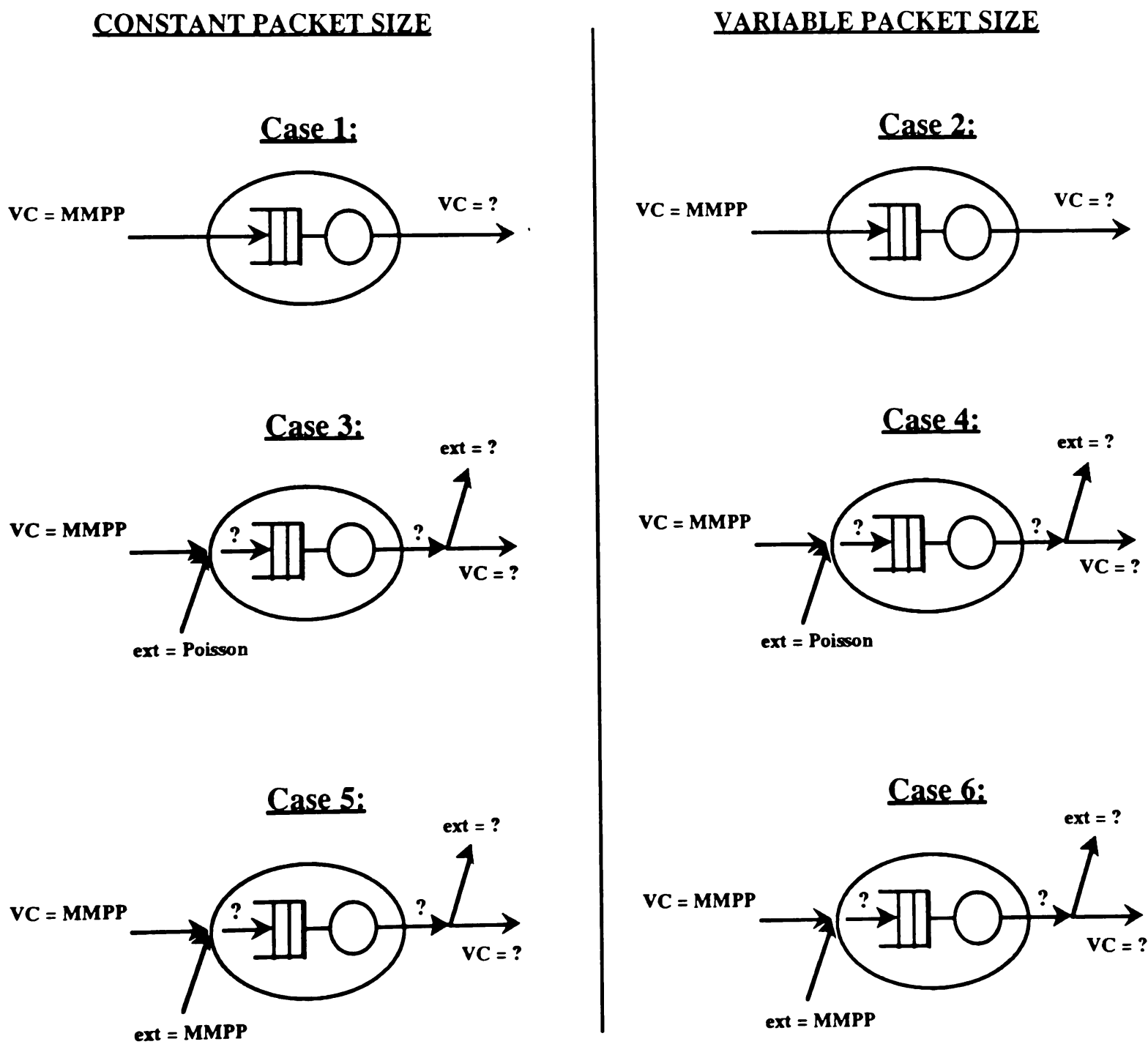

Figure A2.1 Cases for measuring the burstiness of inter-departure times for different packet lengths and input processes

Case 6:

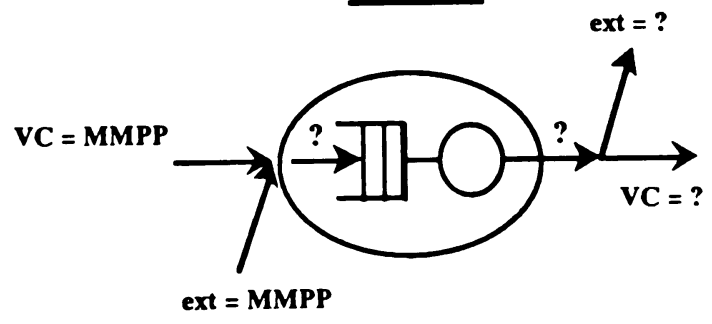


We assume that the network supports variable size packets (like the "Megacell' network used in the study). In each case we focus on measuring burstiness of the departure process and compare the results with the burstiness of the arrival process. As a measure of burstiness we use, again, the square coefficient of variation $\left(c^{2}\right.$, as defined by the equation $/ 16 /$, Appendix C).

The results are derived for the fixed-size (480-byte) packets and variablesize packets (the same 'variable' packet length distribution used throughout the study ${ }^{28}$ ). In the case study 1,3 and 5 we assume fixed-size packets. In study 2,4 and 6 we assumes 'variable' packet sizes.

Each network (queue) has two arrival streams: the 'VC-traffic' and the 'External traffic', as in the topologies 1-4. The VC-traffic is always chosen to be a 'bursty' traffic and it is modeled with an MMPP. The External traffic is varied: none, Poisson, MMPP.

The question marks in figure A2.1 denote the locations where we measure the burstiness of the inter-arrival- and inter-departure times. Our primary interest is in determining the effect of packet lengths and burstiness of the External-traffic on the burstiness of the inter-departure times for the VCand composite (VC- and External) traffic.

The server in each network is assumed to have the capacity of $100 \mathrm{Mbps}$. The total arrival rate from VC- and External traffic in each case is 60 Mbps (hence, the network utilizations are always $60 \%$ ).

The parameters used in the simulation are summarized in Table A2.1. The total MMPP arrival rate, $\lambda$, and the arrival rates in state 1 and $2, \lambda_{1}$ and $\lambda_{2}$ respectively, are kept constant. In each case $\beta$ is varied to achieve different values for burstiness of the arrival process. Given $\lambda, \lambda_{1}, \lambda_{2}$, the $\alpha$ parameters are determined from the eqation /17/, Appendix C (after solving eqn.17 per $\alpha$ ) for each value of $\beta$.

In the case where External arrivals are MMPP (case study 5 and 6) the MMPP parameters are the same as for the VC-MMPP.

Figure A2.2 shows the VC- and External-MMPP arrivals in the case study 5 and 6.

28 'variable' packet length distribution is shown in figure 5, section 3.7 


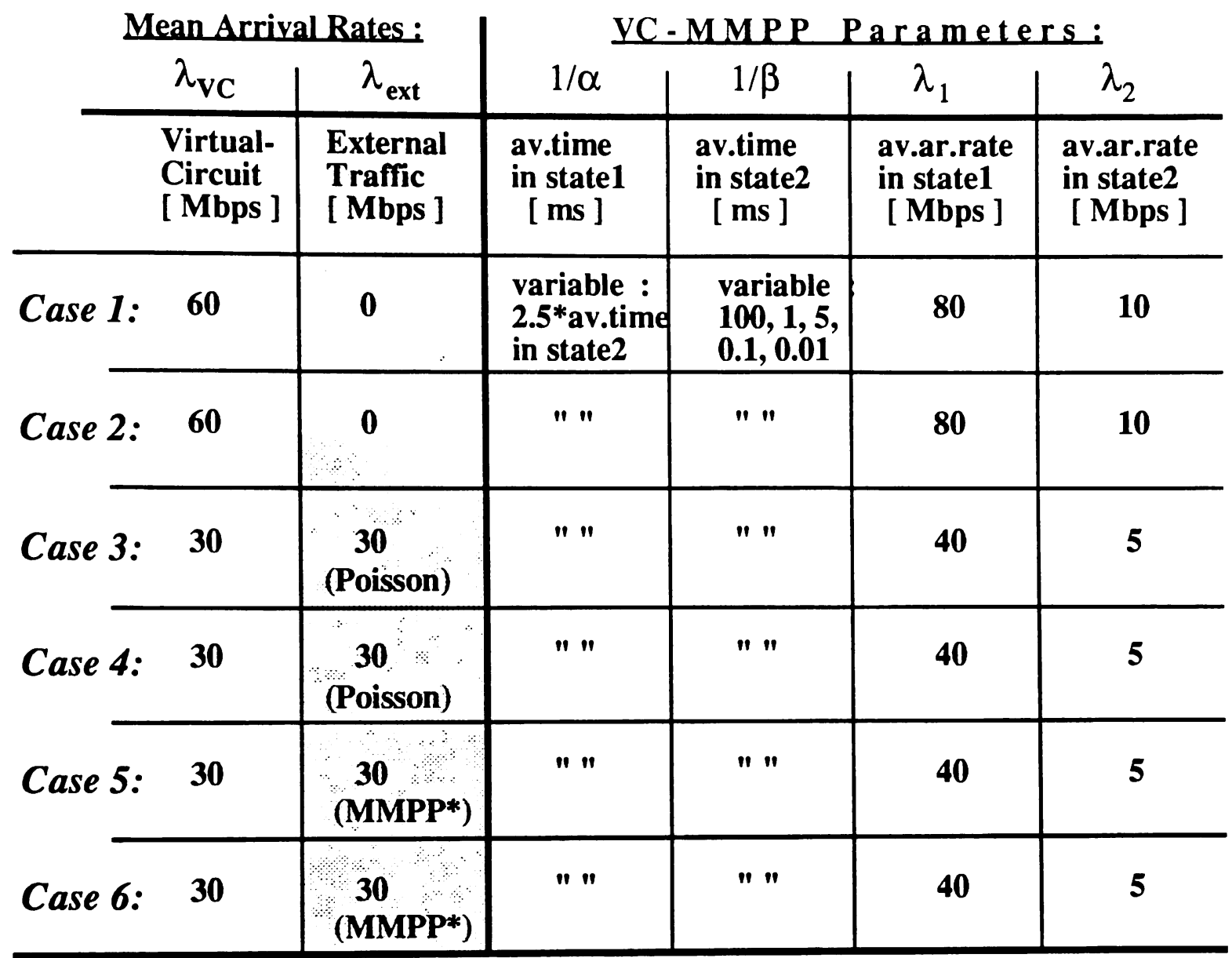

* External arrivals MMPP has the same parameters as the VC-MMPP

Table A2.1 Simulation parameters for the arrival processes total arriv. rate $=60 \mathrm{Mbps}$

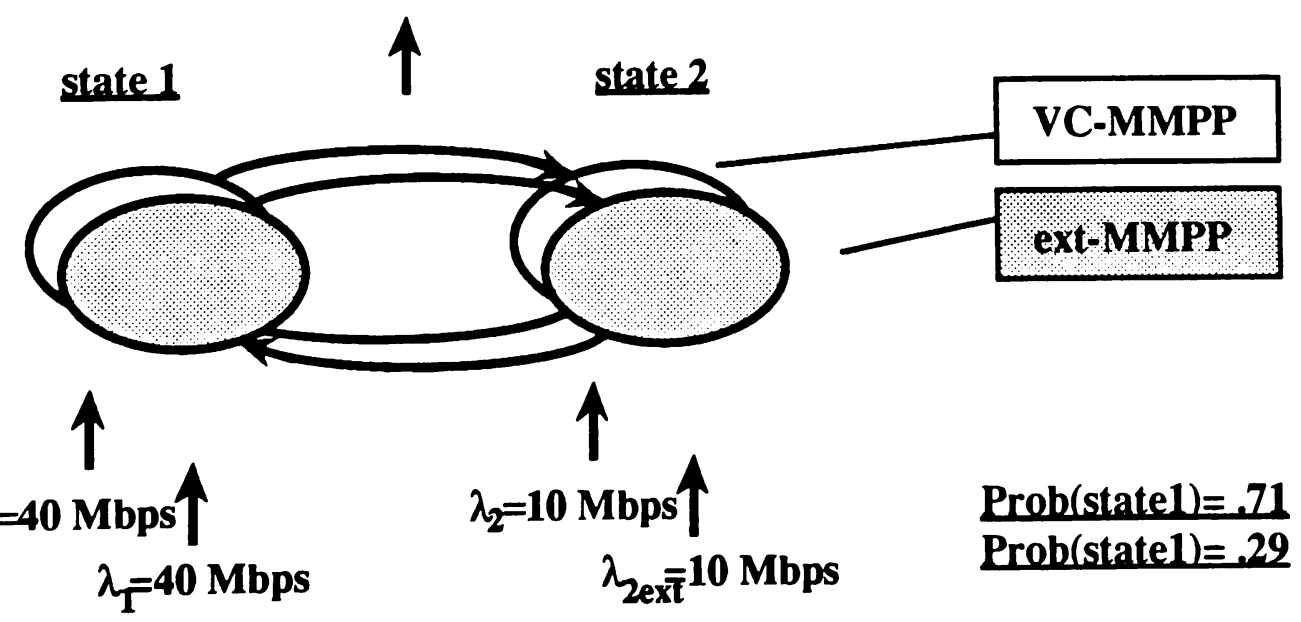

Figure A2.2. VC- and External MMPPs in the case 5 and 6 
Comments on the graphs:

All curves show the burstiness, $c^{2}$, vs. the transition rate from state 2 to state $1, \beta^{29}$. Since in our case $\alpha$ and $\beta$ are linearly related, the small values for $\beta$ lead to small values for $\alpha^{30}$. Also, the small values for $\alpha$ and $\beta$ lead to high burstiness. On the other hand, very large $\beta$ (and, in our case, large $\alpha$ ) cause the MMPP $c^{2}$ to converge to one,.as will be seen in all the graphs $^{31}$.

\section{Discussion of the results:}

The case 1 and 2 enable us to draw a clear conclusion about the effect of packet length on the burstiness of the output traffic. (There are no external arrivals, and therefore, there is no influence on burstiness of the interdeparture times from the external traffic.).

Figure A2.3 and A2.4 show the $c^{2}$ of the inter-arrival times of the VCMMPP and the $c^{2}$ of the inter-departure times for the case 1 and 2. The burstiness of the inter-departure times is much lower in the case of constant packet sizes (case 1), particularly for the case of high burstiness of interarrival times. The explanation for this is that during the bursts, there are a large number of packets in the queue and the inter-departure times are equal to the service time of the packets, since the server is constantly utilized.

On the other hand, in the case of 'variable' packet lengths, burstiness is almost unchanged after the server. Even if the server is constantly utilized during the bursts, the variable packet lengths account for the variability in the inter-departure times and the departure burstiness is not reduced. Our results show that the burstiness of the inter-departure times is slightly reduced in the area of high burstiness of inter-arrival times. In the area of low burstiness (e.g. for the $\beta$ value of 100 ), the burstiness even slightly increased.

29 Reacall that in this study we vary burstiness by varying the $\beta$ values.

30 For the given values of $\lambda, \lambda_{1}, \lambda_{2}$, shown in table A2.1, $\alpha$ turns out to be $.4 \beta$. Hence, $1 / \alpha=2.51 / \beta$, meaning that in all our MMPP cases the average time in the 'high' arrival state (state 1) is 2.5 times longer than the average time in the 'low' arrival state (state 2). 31 See eqation 16, Appendix C 


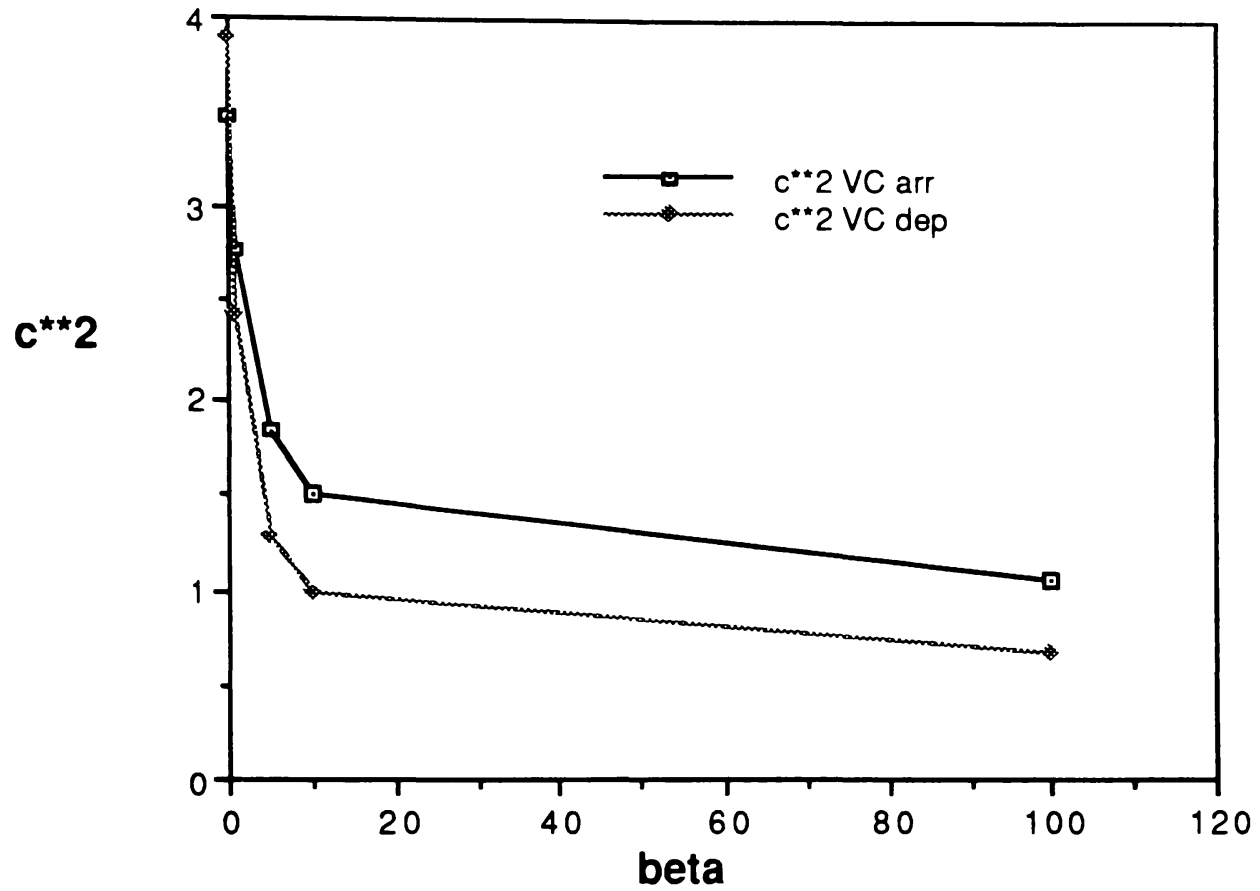

Figure A2.3. CASE 1: $\mathrm{c}^{2}$ of the arrival process and departure process (constant packet size)

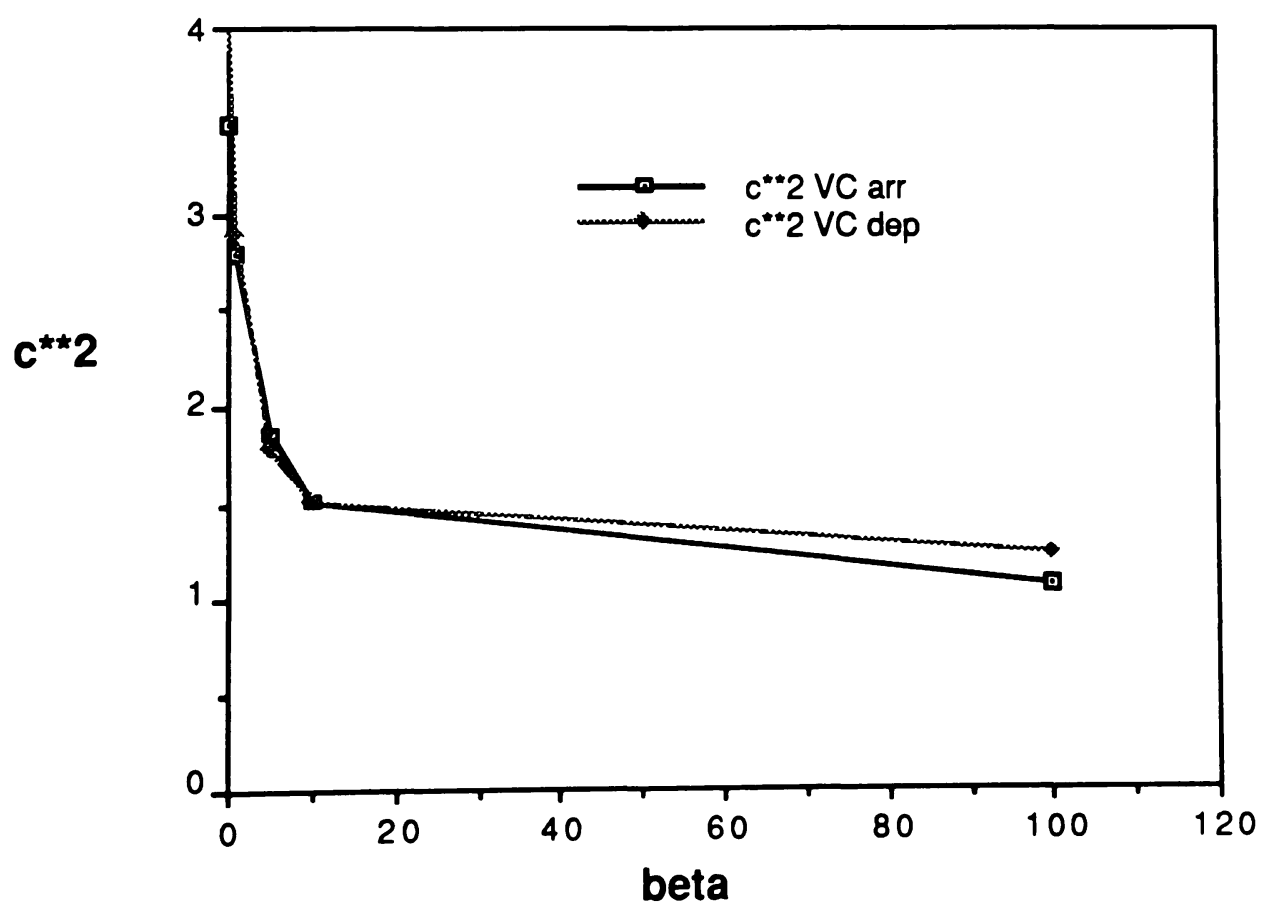

Figure A2.4. CASE 2: $\mathrm{c}^{2}$ of the arrival process and departure process ('variable' packet size) 
In the case 3 and 4 we introduce the Poisson external arrivals. Now we evaluate the $c^{2}$ of the VC-, External and composite arrivals and departures.

Figures A2.5 and A2.6 show the $c^{2}$ of the arrivals, and, figures A2.7 and A2.8 show the $c^{2}$ of the departures for the case 3 . In this case we see, again, the effect of the constant packet size on the burstiness of the output process. Notice that all the departure $c^{2}$ curves have lower $c^{2}$ values. The Poisson arrival stream (external traffic) became even more deterministic ( $c^{2}$ is lower than 1 ). The $c^{2}$ of the composite departure process became also more deterministic.

Now, let us compare the above results with the results for the case 4 (figures A2.7 and A2.8).

In the case 4 the results are somewhat surprizing. We notice that in all the departure curves, $c^{2}$ is above 1 . The Poisson arrival stream became more bursty. Also, the departure process for the composite traffic became much more bursty. This leads to an important observation: If a burtsy stream is multiplexed with a Poisson stream, the resulting departure process may not necessarily 'smooth-out'. On the contrary, it may even get more bursty! The above result was a surprize to us, as we said, at least in the first moment ${ }^{32}$.

The results for the cases 5 and 6 were similar to the above cases. Namely, the $c^{2}$ of the departure process was greatly influenced by the packet length distribution. In figures A2.9 and A2.10 we show the $c^{2}$ of the arrival and departure processes for the case of the composite traffic. In the case 5 (fixed packet size), the $c \stackrel{2}{\text { of the departure process became significantly }}$ $\underline{\text { lower. In the case } 6 \text { (variable packet size), the } c 2 \text { of the departure process }}$ became even slightly higher.

32"The intuition is good, as long as it is correct" 


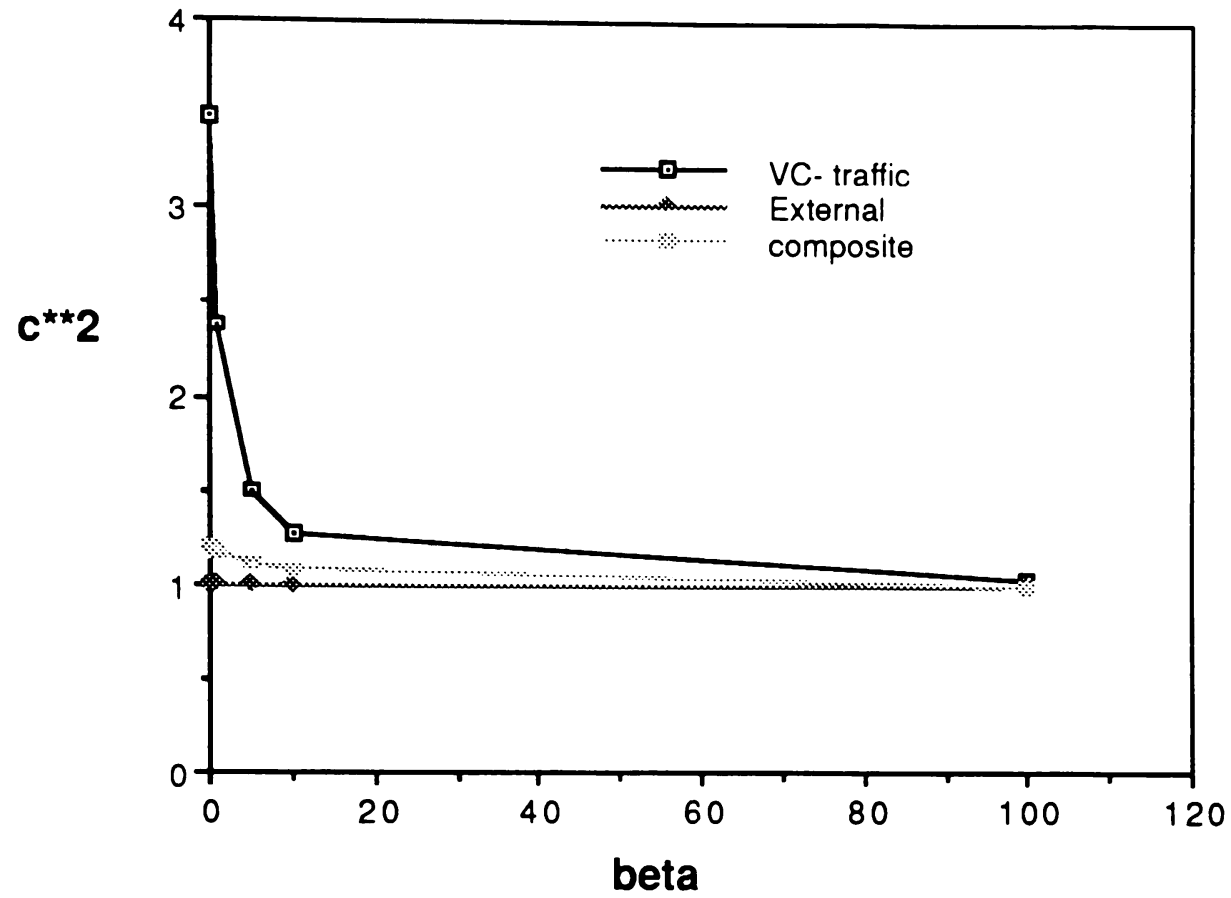

Figure A2.5. CASE 3: $c^{2}$ of the VC-, external and composite arrival process (constant packet size)

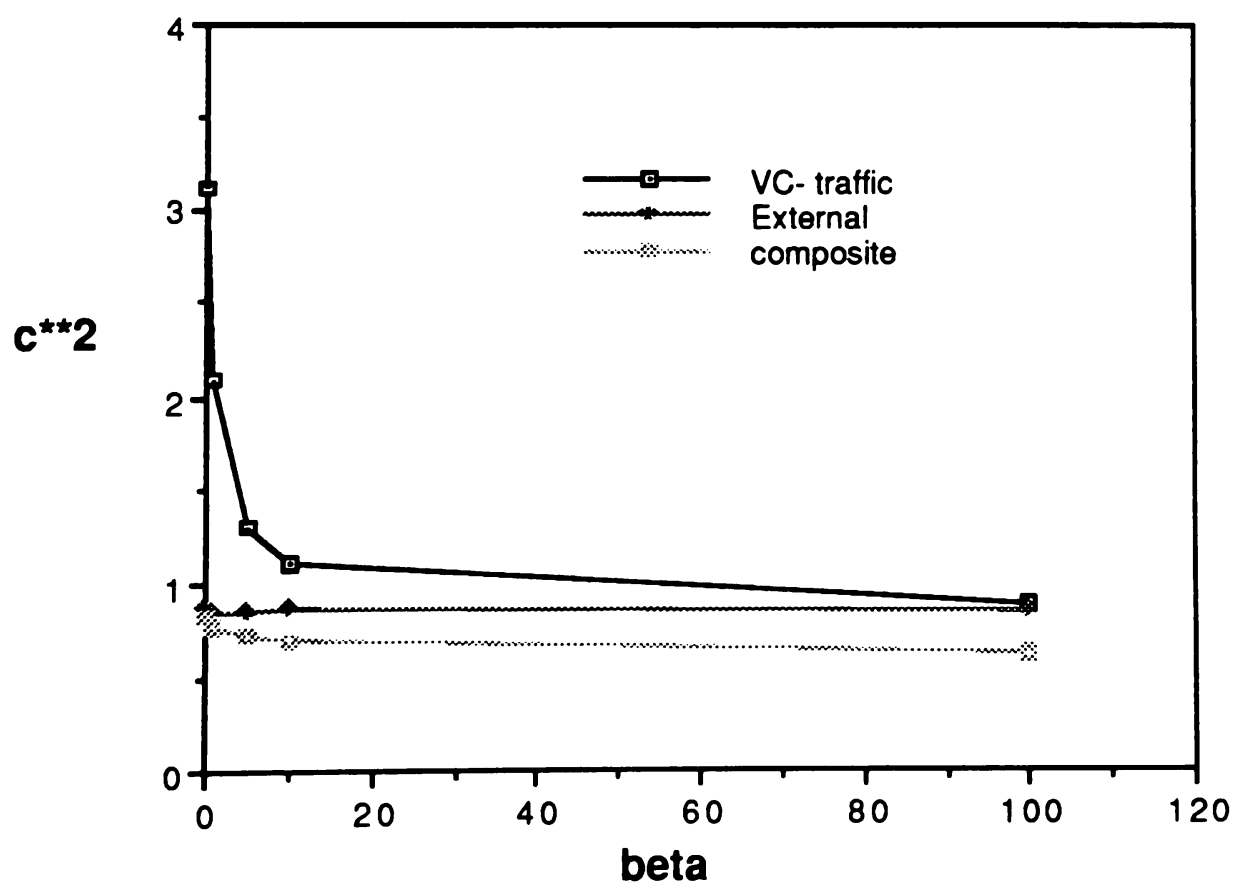

Figure A2.6. CASE 3: $\mathrm{c}^{2}$ of the VC-, external and composite departure process (constant packet size) 


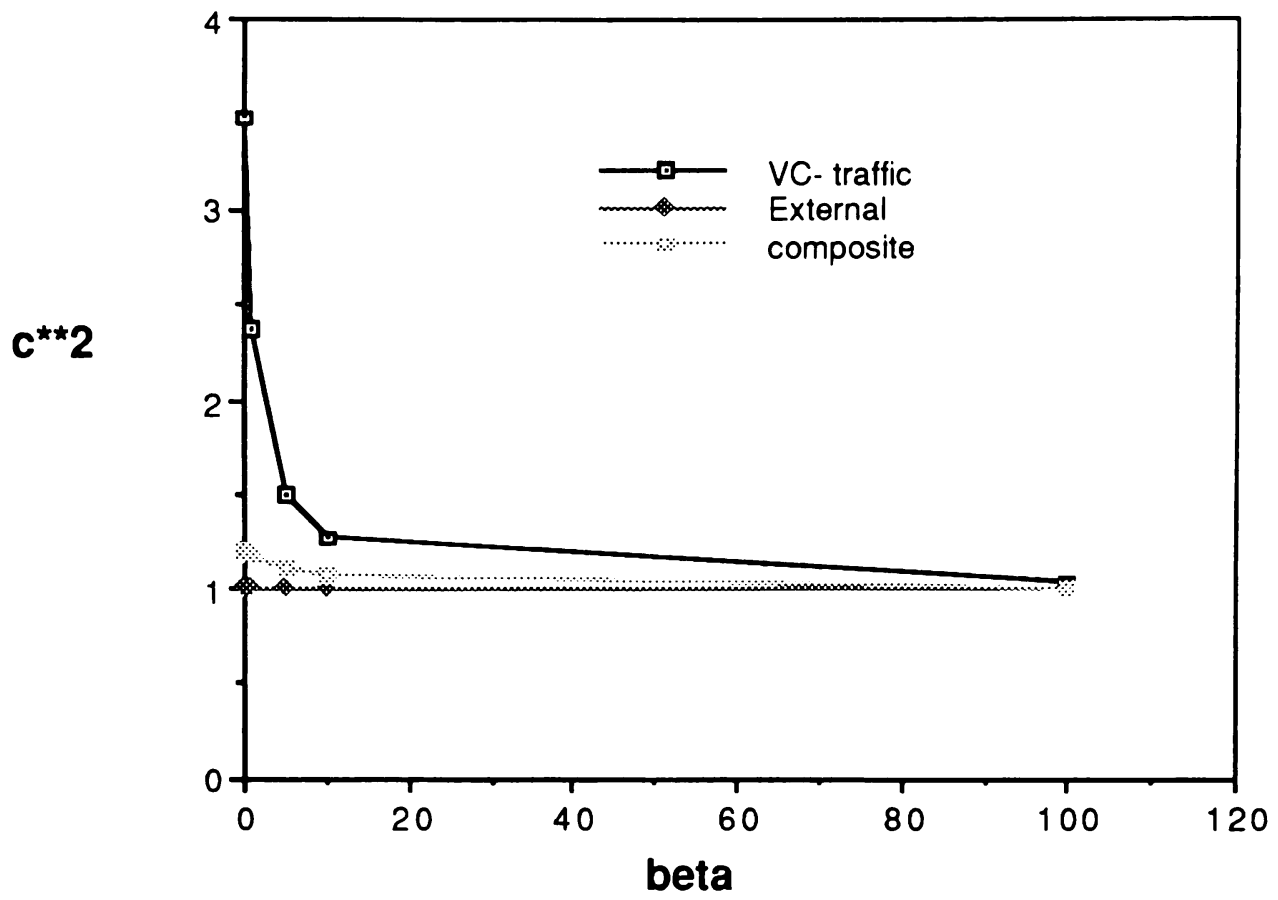

Figure A2.7. CASE 4: $c^{2}$ of the VC-, external and composite arrival process (variable packet size)

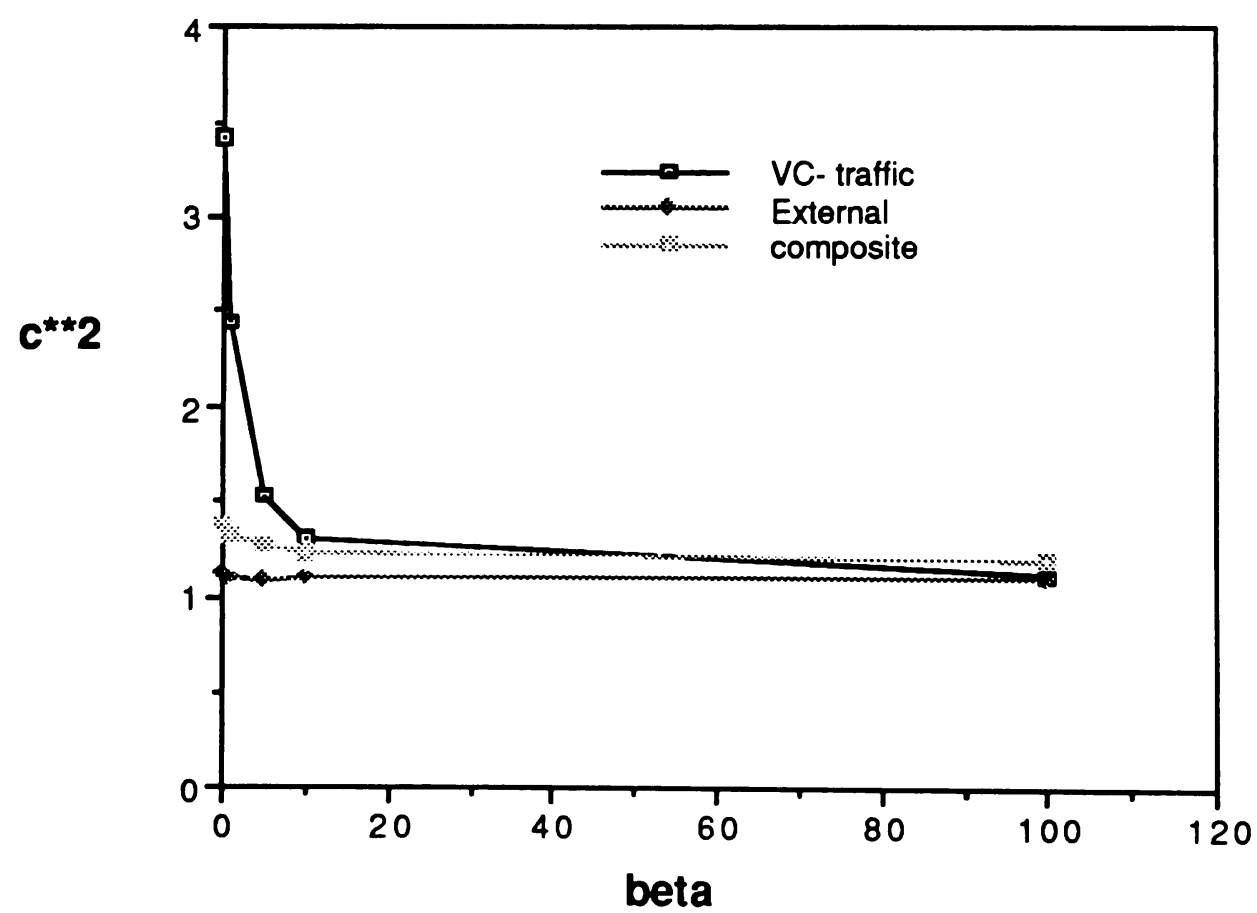

Figure A2.8. CASE 4: $\mathrm{c}^{2}$ of the VC-, external and composite departure process (variable packet size) 


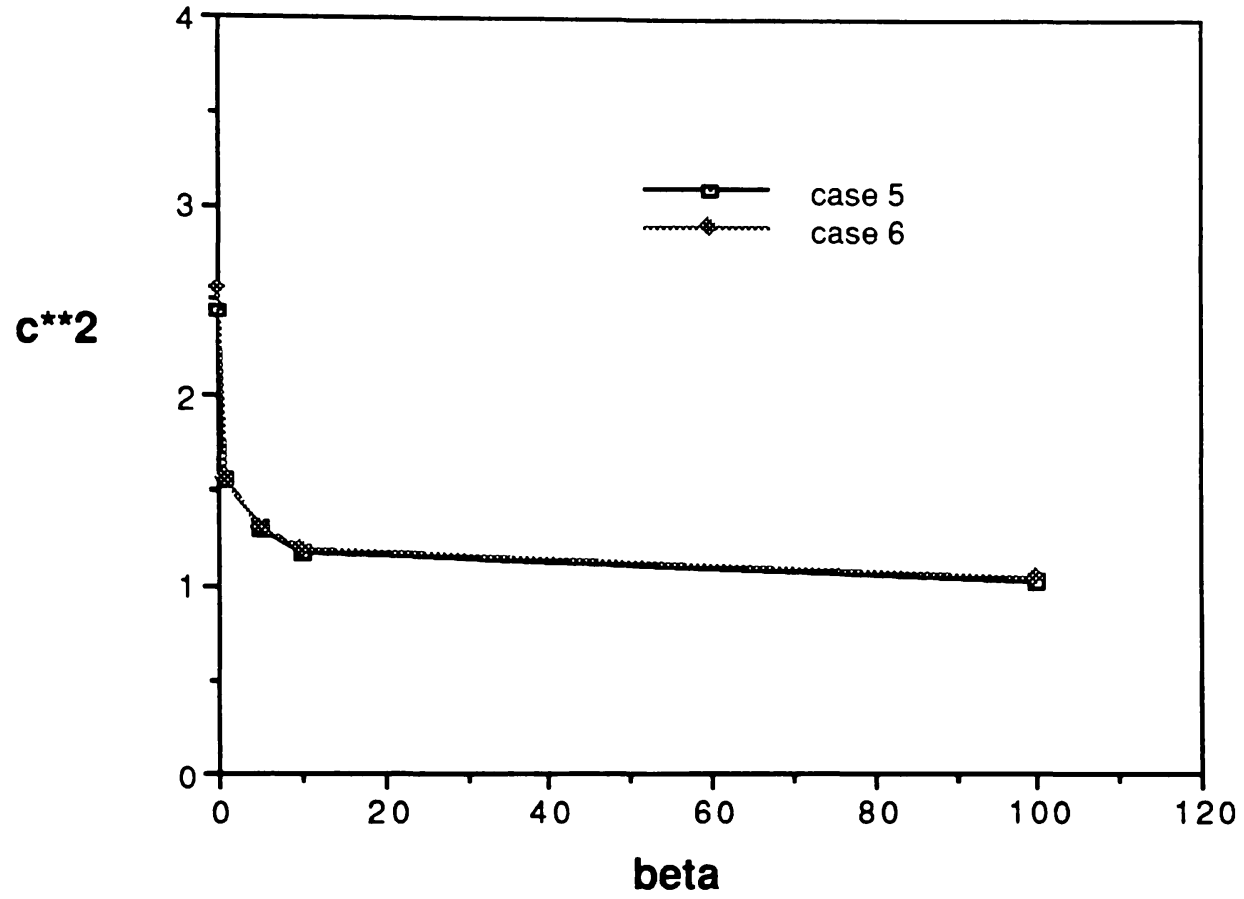

Figure A2.9. CASE 5 and 6: $c^{2}$ of the composite arrival process

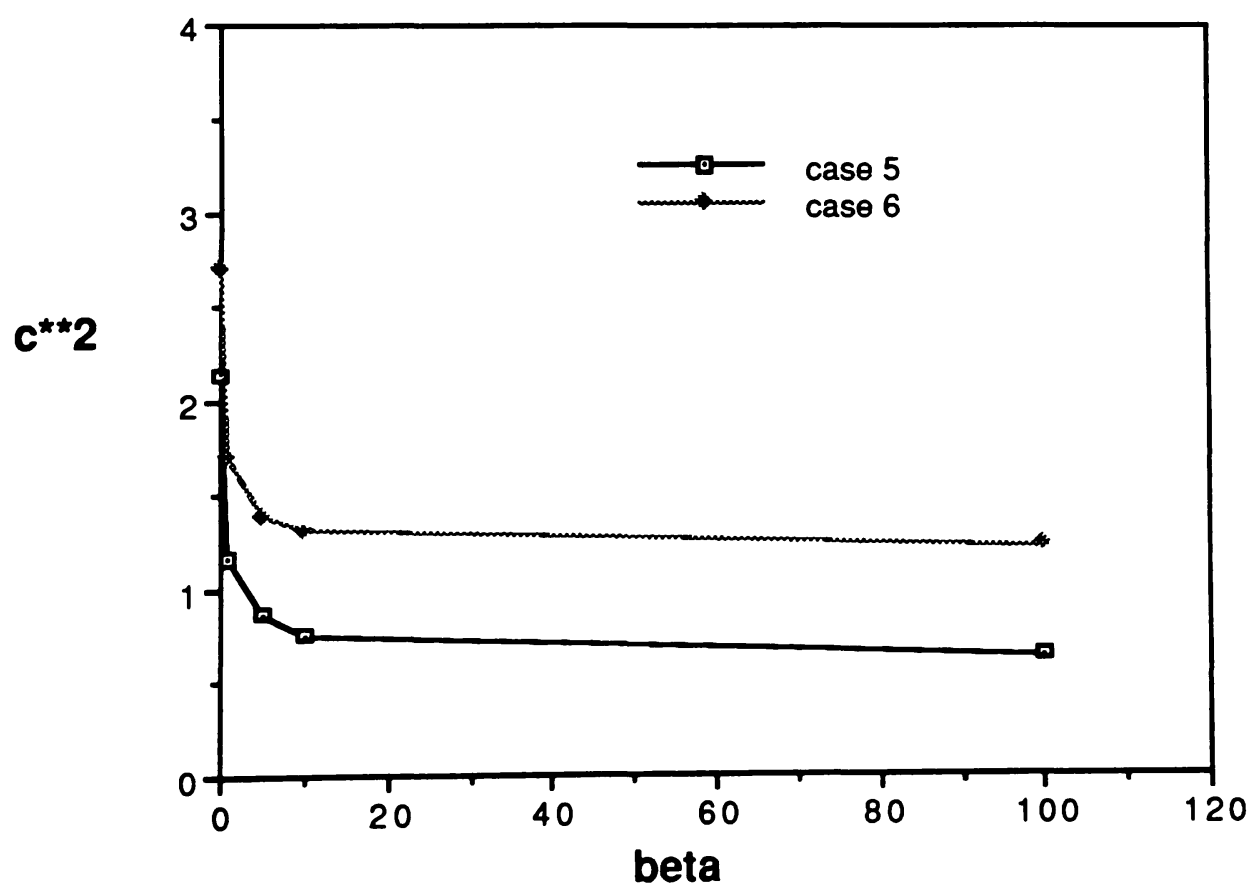

Figure A2.10. CASE 5 and 6: $\mathrm{c}^{2}$ of the composite departure process 


\section{APPENDIX C}

Derivation of pdf of Inter-arrival times and Squared Coefficient of Variation ( $c^{2}$ ) of Inter-arrival times for the two-state Markov Modulated Poisson Process

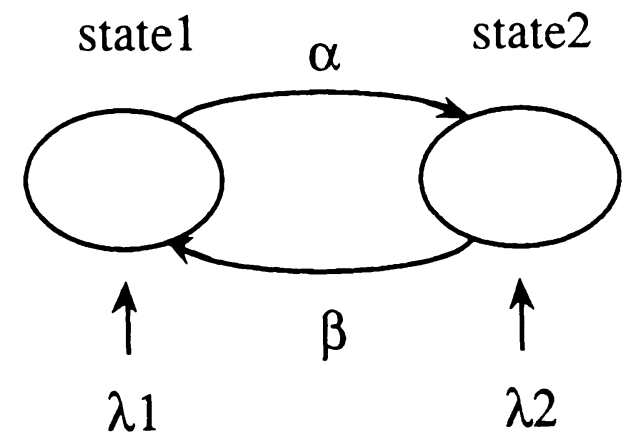

Figure A3.1. Two-state Markov Modulated Poisson Process (MMPP)

Let $\lambda_{1}$ and $\lambda_{2}$ be the arrival rates in state 1 and 2 ; let $\alpha$ be the transition rate from state 1 to state 2 and $\beta$ be the transition rate from state 2 to state 1 , as shown in Figure A3.

Probabilities of MMPP being in state 1 and $2, \pi_{1}$ and $\pi_{2}$ respectively, are given by:

$$
\pi_{1}=\frac{\beta}{\alpha+\beta} \quad \text { and } \quad \pi_{2}=\frac{\alpha}{\alpha+\beta}
$$

Now, let us consider that MMPP is initially in state 1 and assume that the last event occured was an arrival at time $t$ (see figure A4). The next event in state 1 can be an arrival or a state change. The probability that the next event is an arrival is:

$$
\pi(\text { arriv. } \mid \text { state } 1)=\frac{\lambda_{1}}{\lambda_{1}+\alpha}
$$

The probability that the next event is a state change is:

$$
\pi(\text { arriv. I state } 1)=\frac{\alpha}{\lambda_{1}+\alpha}
$$


Let us denote the time until the next event in state 1 with the random variable $\mathrm{T}_{1}$ and the time until the next arrival in state 1 with the random variable $t_{1}$.

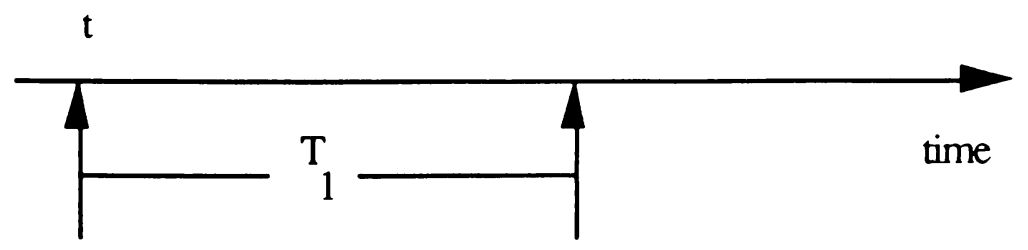

arrival

next event:

arrival or state change

Figure A4. Possible events in state 1 after an arrival in state 1

Hence, the time until the next arrival in state 1 is equal to:

$$
\mathrm{t}_{1}=\left\{\begin{array}{ccc}
\mathrm{T}_{1} & \text { with prob. } & \frac{\lambda_{1}}{\lambda_{1}+\alpha} \\
\mathrm{t}_{2}+\mathrm{T}_{1} & \text { with prob. } & \frac{\alpha}{\lambda_{1}+\alpha}
\end{array}\right.
$$

Consequently, the time until the next arrival in state 2 is equal to:

$$
\mathrm{t}_{2}=\left\{\begin{array}{ccc}
\mathrm{T}_{2} & \text { with prob. } & \frac{\lambda_{2}}{\lambda_{2}+\beta} \\
\mathrm{t}_{1}+\mathrm{T}_{2} & \text { with prob. } & \frac{\beta}{\lambda_{2}+\beta}
\end{array}\right.
$$

By applying the Laplace transform to /5,6/ we obtain the probability density functions for inter-arrival times $t_{1}$ and $t_{2}, A_{1}(s)$ and $A_{2}(s)$ respectively as:

$$
\begin{aligned}
& \mathrm{A}_{1}(\mathrm{~s})=\frac{\lambda_{1}+\alpha}{\lambda_{1}+\alpha+\mathrm{s}} \frac{\lambda_{1}}{\lambda_{1}+\alpha}+\left(\frac{\lambda_{1}+\alpha}{\lambda_{1}+\alpha+\mathrm{s}}+\mathrm{A}_{2}(\mathrm{~s})\right) \frac{\lambda_{1}}{\lambda_{1}+\alpha} \\
& \mathrm{A}_{2}(\mathrm{~s})=\frac{\lambda_{2}+\beta}{\lambda_{2}+\beta+\mathrm{s}} \frac{\lambda_{2}}{\lambda_{2}+\beta}+\left(\frac{\lambda_{2}+\beta}{\lambda_{2}+\beta+\mathrm{s}}+\mathrm{A}_{1}(\mathrm{~s})\right) \frac{\lambda_{2}}{\lambda_{2}+\beta}
\end{aligned}
$$

Solving /7,8/ per $A_{1}(s)$ and $A_{2}(s)$ we obtain: 


$$
\begin{aligned}
& \mathrm{A}_{1}(\mathrm{~s})=\frac{\alpha \lambda_{2}+\lambda_{1}\left(\beta+\lambda_{2}+\mathrm{s}\right)}{\left(\alpha+\lambda_{1}+\mathrm{s}\right)\left(\beta+\lambda_{2}+\mathrm{s}\right)-\alpha \beta} \quad \text { and } \\
& \mathrm{A}_{2}(\mathrm{~s})=\frac{\beta \lambda_{1}+\lambda_{2}\left(\alpha+\lambda_{1}+\mathrm{s}\right)}{\left(\beta+\lambda_{2}+\mathrm{s}\right)\left(\alpha+\lambda_{1}+\mathrm{s}\right)-\alpha \beta}
\end{aligned}
$$

Now, having the inter-arrival time pdf for each of the two states, the Laplace transform of the pdf for the two-state MMPP, A(s), is obtained by multiplying individual pdf's by the probability of having an inter-arrival time from the particular pdf (i.e. probability that MMPP is in state 1 and state 2).

$$
\mathrm{A}(\mathrm{s})=\mathrm{A}_{1}(\mathrm{~s}) \pi_{1}+\mathrm{A}_{2}(\mathrm{~s}) \pi_{2}
$$

After substituting $/ 1,2 /$ for $\pi_{1}$ and $\pi_{2}$ and simplifying, the Laplace transform of the pdf of inter-arrival times for the two-state MMPP is given by:

$$
A(s)=\frac{1+\frac{\left(\beta \lambda_{1}{ }^{2}+\alpha \lambda_{2}{ }^{2}\right) s}{\left(\beta \lambda_{1}+\alpha \lambda_{2}\right)\left(\beta \lambda_{1}+\alpha \lambda_{2}+\lambda_{1} \lambda_{2}\right)}}{1+\frac{\left(\alpha+\beta+\lambda_{1}+\lambda_{2}\right) s+s^{2}}{\beta \lambda_{1}+\alpha \lambda_{2}+\lambda_{1} \lambda_{2}}}
$$

The squared coefficient of variation of inter-arrival times, $\mathrm{c}^{2}$, is defined as the variance over the mean square of the inter-arrival times. Let us denote the MMPP-inter-arrival times by $\tau_{1}$ and the variance and mean of interarrival times as $\operatorname{var}\left(\tau_{1}\right)$ and $E\left(\tau_{1}\right)$. Hence,

$$
c^{2}=\frac{\operatorname{var}\left(\tau_{l}\right)}{E^{2}\left(\tau_{l}\right)}=\frac{E\left(\tau_{l}^{2}\right)-E^{2}\left(\tau_{l}\right)}{E^{2}\left(\tau_{l}\right)}
$$

We find now the first two moments of the MMPP-pdf, given in /12/. The mean inter-arrival time, $\mathrm{E}\left(\tau_{\mathrm{l}}\right)$, turns out to be:

$$
E\left(\tau_{1}\right)=\frac{\alpha+\beta}{\beta \lambda_{1}+\alpha \lambda_{2}}
$$


The second moment is equal to:

$$
\mathrm{E}\left(\tau_{\mathrm{l}}^{2}\right)=\frac{2\left((\alpha+\beta)^{2}+\mathrm{al}_{1}+\mathrm{bl} \mathrm{l}_{2}\right)}{\left(\mathrm{bl}_{1}+\mathrm{al}_{2}+\mathrm{l}_{1} \mathrm{l}_{2}\right)}
$$

After inserting /14,15/ into /13/ and simplifying we obtain the squared coefficient of variation of inter-arrival times of a two-state MMPP:

$$
c^{2}=1+\frac{2 \alpha \beta\left(\lambda_{1}-\lambda_{2}\right)^{2}}{\left(\beta \lambda_{1}+\alpha \lambda_{2}+\lambda_{1} \lambda_{2}\right)(\alpha+\beta)^{2}}
$$

Notice, for $\lambda_{1}=\lambda_{2}$, the two-state MMPP reduces to a Poisson process and the $c^{2}=1$. Analogously, in the limiting cases, where $\alpha$ or $\beta \rightarrow 0$ (i.e. the mean time MMPP is in state 1 or 2 is very long), the $c^{2}->1$.

Equation /16/ enables us to have full control over the selection of the MMPP parameters in order to obtain the desired burstiness of the arrival process.

Additional comment:

The total MMPP arrival-rate, $\lambda$, is given by:

$$
\lambda=\lambda_{1} \pi_{1}+\lambda_{2} \pi_{2}
$$




\section{APPENDIX D}

An approximate analytical result for the square coefficient of variation of inter-departure times for an MMPP/M/1 queue

In this derivation we make use of the result obtained for the inter-arrival time distribution for the MMPP, described in the Appendix $C$, and the inter-departure time distribution for a GI/M/1 queue, obtained by Daley [31].

The analysis is approximate since, to use Daley's result, the inter-arrival times have to be independent. In the MMPP, the arrivals are correlated.

Let us denote the inter-departure times by $\tau_{\delta}$. From [31], the Laplace transform of the inter-departure time distribution for an $\mathrm{GI} / \mathrm{M} / 1$ queue is given by:

$$
D(s)=\frac{\mu}{\mu+s} \frac{\sigma s-\mu(1-\sigma) A(s)}{s-\mu(1-\sigma)},
$$

where $\sigma$ is the unique root in $0<\sigma<1$ of

$$
\sigma=\mathrm{A}(\mu(1-\sigma))
$$

$\mu$ is the service rate, and $A(s)$ is the inter-departure time distribution.

After substituting the result for $A(s)$ (eqn. /12/, Appendix C) into /1/, we obtain:

$$
D(s)=\frac{\mu}{\mu+s} \frac{1+\frac{\left(\beta \lambda_{1}{ }^{2}+\alpha \lambda_{2}\right)^{2} s}{(\beta s-\mu(1-\sigma))}}{1+\frac{\left.\left(\alpha+\beta+\lambda_{1}+\lambda_{2}\right) s+s_{2}\right)\left(\beta \lambda_{1}+\alpha \lambda_{2}+\lambda_{1} \lambda_{2}\right)}{\beta \lambda_{1}+\alpha \lambda_{2}+\lambda_{1} \lambda_{2}}}
$$

The $c^{2}$ of the inter-departure times is given by:

$$
c^{2}=\frac{\operatorname{var}(\tau \delta)}{E^{2}(\tau \delta)}=\frac{E\left(\tau_{\delta}^{2}\right)-E^{2}(\tau \delta)}{E^{2}(\tau \delta)}
$$


Hence, we find next the first two moments of $D(s)$. The mean interdeparture time, $\mathrm{E}\left(\tau_{\delta}\right)$, is given by:

$$
E\left(\tau_{\delta}\right)=\frac{\alpha+\beta}{\beta \lambda_{1}+\alpha \lambda_{2}}
$$

This result is the same as the mean inter-arrival time for an MMPP, as expected.

After obtaining the equation for the second moment ${ }^{33}$ and then substituting the first and second moment into /4/ and simplifying, we finally obtain the $c^{2}$ for the inter-departure time distribution as:

$$
\begin{aligned}
& c^{2}=\frac{2\left(\beta \lambda_{1}+\alpha \lambda_{2}\right)\left((\alpha+\beta)^{2}+\alpha \lambda_{1}+\beta \lambda_{2}\right)}{(\alpha+\beta)^{2}\left(\beta \lambda_{1}+\alpha \lambda_{2}+\lambda_{1} \lambda_{2}\right)}+ \\
& \frac{2\left(\beta \lambda_{1}+\alpha \lambda_{2}\right)\left(\beta \lambda_{1}+\alpha \lambda_{2}-\sigma(\alpha+\beta) \mu\right)}{(1-\sigma)(\alpha+\beta)^{2} \mu^{2}}-1
\end{aligned}
$$

Notice that $c^{2}$ of the inter-departure times is a function of $\sigma$. Therefore, we now have to determine the root $\sigma$ from the equation $/ 2 /$. The analytical solution to the equation /2/ leads to a very long expression. Therefore, we find values for $\sigma$ numerically for each particular set of MMPP parameters $\left(\alpha, \beta, \lambda_{1}, \lambda_{2}\right)$ and the service rate $\mu$.

We assume an MMPP arrival process with the characteristics as used in the case 2, Appendix $\mathrm{C}$.

After obtaining the $\sigma$ values for the desired MMPP process, the numerical values are substituted in /6/ and the corresponding inter-departure time $c^{2}$ values are obtained.

In figure A4.1 we show the analytical versus simulation results for the inter-departure time $c^{2}$ for the case 2 . We can observe that for the case of the 'variable' packet length distribution and MMPP input process, the approximate analytical results for the $c^{2}$ of the departure process are very close to the simulation results. The small difference in the results can be explained through the fact that the 'variable' packet length distribution is not an exponential distribution, which is assumed in the analytical model.

33 The derivations are obtained using Mathematica on the Macintosh computer. The equation for the second moment turns out to be very long to be printed here. 


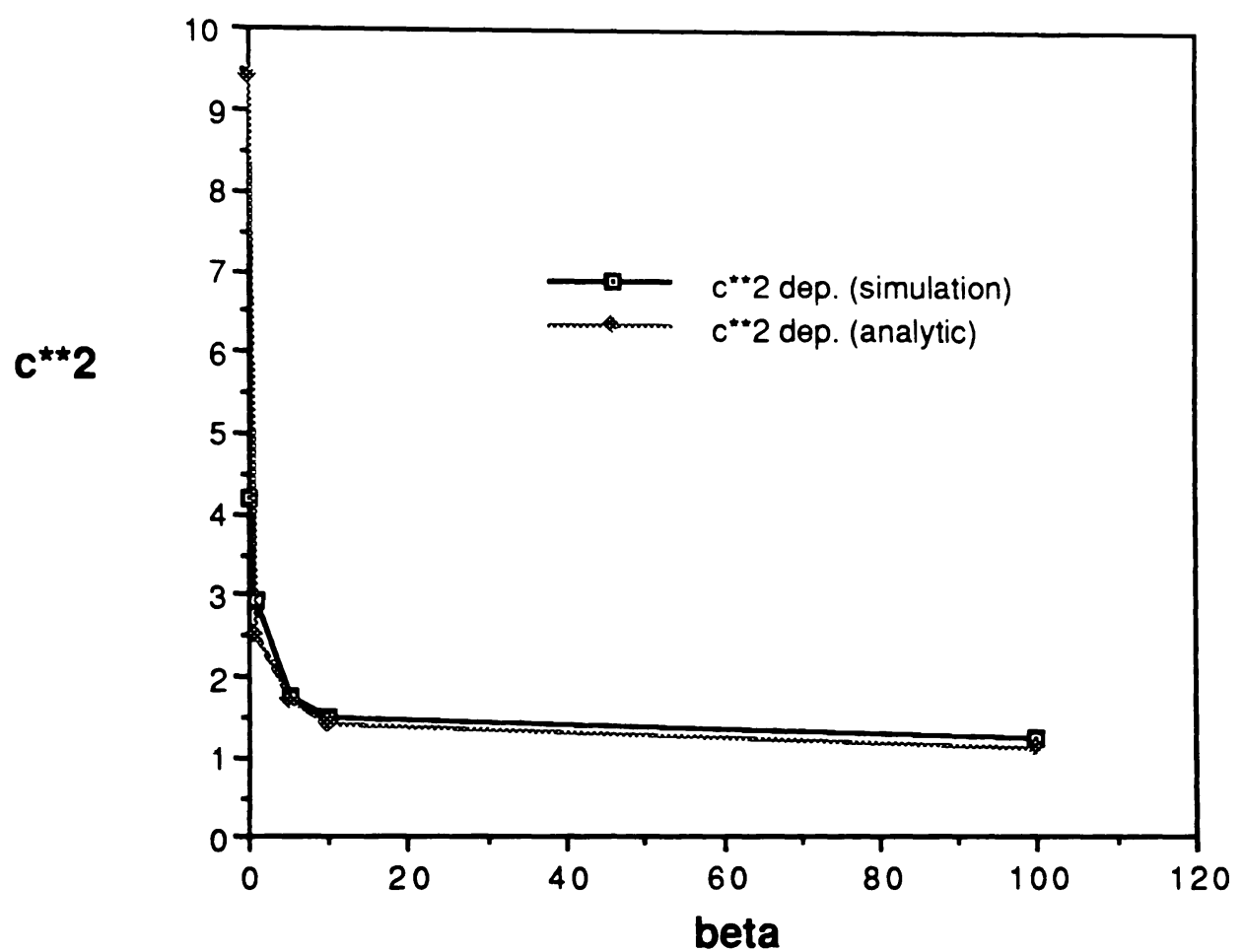

Figure A4.1 Analytical and Simulation results for the $c^{2}$ of inter-departure times for the case 2, Appendix C 
The Bottom Line:

From the presented diagrams it becames obvious that for the cases of bursty arrivals and variable size packets one cannot conclude that the burstiness of the network traffic is going to become lower after the traffic has passed one (or more) network nodes.

This indicates that the external arrivals (aggregate network traffic) in a variable length cell broadband network should not be modeled as Poisson.

The above results give a strong motivation to look into the departure processes in more detail and try to obtain valuable analytical solutions. 